\title{
Julia Set As A Martin Boundary
}

\author{
Dissertation \\ zur Erlangung des Doktorgrades der \\ Mathematisch-Naturwissenschaftlichen Fakultäten der \\ Georg-August-Universität zu Göttingen
}

\author{
Vorgelegt von \\ Md. Shariful Islam \\ aus \\ Munshigonj, Bangladesch
}

Göttingen 2010 
D7

Referent: Prof. Dr. Laurent Bartholdi

Korreferent: Prof. Dr. Preda Mihailescu

Tag der mündlichen Prüfung: 5. Juli 2010 


\section{$\underline{\text { Acknowledgment }}$}

It is my great pleasure to express my indebtedness and deep sense of gratitude to Prof. Dr. Laurent Bartholdi for supervising my PhD work. I am also very grateful to Prof. Dr. Manfred Denker for his assistance and encouragement particularly with chapter 1-3.

I would like to thank Dr. Manuel Stadlbauer, Dr. Sachar Kablutschko, Tania Garfias Macedo and Achim Wuebker for several stimulating discussion. I acknowledge the financial support of Gottlieb Daimler and Karl Benz Foundation with deep appreciation which made this project possible. I am also very much thankful and indebted to all the members of my family who inspired me every possible way though we are far apart by time and distance. My very special thanks go to Prof. Dr. Hans Strasburger for his encouragement and proofreading of my thesis. I am also indebted to Silke Rossmann for her inspiration and help as I went through the very ups and downs of life.

I would like to thank both the secretaries, Ms. Carmen Barann and Ms. Hertha Zimmer, Department of Mathematics, University of Göttingen, and all of my friends and colleagues who have extended their helping hands every now and then and have taken every possible effort to create a nice environment for smooth learning. 


\section{Contents}

1 Introduction 1

2 Preliminaries $\quad 7$

2.1 Rational Map and its Julia set . . . . . . . . . . . . . . . 7

2.2 Self-similar Sets . . . . . . . . . . . . . . . . . . . 10

2.3 Results from Potential Theory . . . . . . . . . . . . . . . . . . 12

2.4 Shift space and Ruelle Operator . . . . . . . . . . . . . . 17

2.5 Entropy Pressure and Gibbs Measure . . . . . . . . . . . . . . . 20

3 Julia Set as a Martin Boundary $\quad 25$

3.1 Markov Chain on the Word Space . . . . . . . . . . . . . . 25

3.2 Martin Kernel and Martin Boundary . . . . . . . . . . . . . . . . . . 29

3.3 Determination of the Martin Kernel . . . . . . . . . . . . . . . . . . . 32

3.4 Symbolic Space and Julia Set . . . . . . . . . . . . . . . . . . 37

3.5 Julia set as a Martin Boundary . . . . . . . . . . . . . . . . . . . . . 40

4 Various Measures on the Julia Set $\quad 47$

4.1 Quasi-invariant Measure on the Julia Set . . . . . . . . . . . . . . . 47

4.2 Gibbs and Equilibrium Measures . . . . . . . . . . . . . . . 53 
4.3 Capacity of the Julia Set and Harmonic Measures . . . . . . . . . . . 61

4.4 An Example with a Rational Map . . . . . . . . . . . . . . . . . 65 


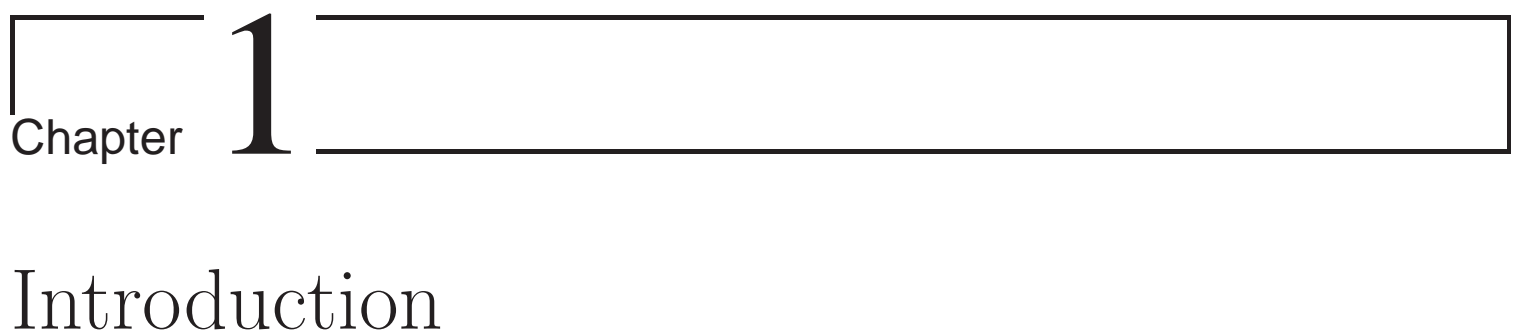

The study of the dynamics of rational maps on the Riemann sphere dates back to the early part of the 20th century and involves the work of Pierre Fatou and Gaston Julia. Iterative dynamical systems had recently appeared at the forefront of mathematics with the work of Henri Poincaré on planetary motion; however, it was the announcement of a competition in 1915 in France that prompted research on the iteration of rational maps [3]. Only a few years before, Paul Montel had begun his fundamental study of normal families of holomorphic functions [46]. Julia won the prize in 1918, and Fatou published his own, nearly identical results a few years later. The two are credited for building the foundations of complex dynamics, and are particularly praised for their clever applications of Montel's theory of normal families [32], [25], [26], [27]. Their work generated a flurry of excitement, but the subject soon fell out of favor.

Complex dynamics became popular in the last twenty years, due in part to the advent of quality computer graphics showing the complicated and beautiful objects which appear naturally through iteration [31]. It was quickly discovered that as a branch of pure mathematics, complex dynamics is rich and tantalizing, most especially for 
its links to other branches of mathematics such as analysis in both one and several variables, potential theory, and algebraic geometry.

The dynamics on the Fatou set are normal (in the sense of Montel), and are well understood whereas the dynamics on the Julia set are quite the opposite - chaotic and unpredictable. The Julia set often turns out to be fractal. Though Fractals were known to mathematicians early in the twentieth century, they were not of much interest then. The situation changed dramatically when Mandelbrot coined the word "fractal" in 1975 and illustrated this mathematical object with striking computer-constructed visualizations. He claimed (see Mandelbrot [41], [42]) that many objects in nature are not well described as collections of smooth components, and are rather better modelled and studied by using the notion of fractals. His proposal was recognized, and a new field of mathematics called "fractal geometry" developed quickly. However, developing a theory of analysis on fractals is a new challenge because of the absence of smooth structures on fractals. For example, one can not define a differential operator like the Laplacian from the classical viewpoint of analysis.

As fractals and chaos are closely related and often coexist, stochastic tools, such as the Markov chain, martingales, or Brownian motion, are well suited for analyzing the dynamics on such regions. It was Poincaré who introduced the probabilistic concept to dynamics. In our case the Markov chain will be used to model the dynamics.

The classical Poisson formula yields an integral representation of a bounded harmonic function in the unit disk in terms of its boundary values. Given a Markov operator $P$ on a state space $X$, we can easily define harmonic functions as invariant functions of the operator $P$, but in order to speak about their boundary values we need a boundary, because no boundary is normally attached to the state space of a Markov chain (as distinct from bounded Euclidean domains common 
for the classical potential theory). One way to overcome this limitation is to find a topological compactification of the state space naturally connected with the Markov operator $P$. That is what was achieved by Martin by constructing the famous Martin boundary and representing superharmonic functions as integrals over the boundary (see Martin [44]). The probabilistic interpretation of Martin's result was proposed by Doob [21]. The most important boundary from a probabilistic and potential theoretic viewpoint is the Martin boundary which describes all positive harmonic and superharmonic functions by integrals on the boundary (see Dynkin [22]). In many cases, this also leads to a solution of the associated Dirichlet problem. It is therefore a natural question how to identify the Martin boundary. One of our main goals is to make a contribution to this identification problem.

The existing proofs of such an identification theorem follow a certain pattern. First one assigns a topological boundary to the paths of the chain and then proves that it coincides with the Martin boundary. The Markov chain will be defined on a state space $\mathcal{W}$, the tree of all finite words over a fixed finite alphabet, and the transitions from one word to another will be positive for certain pairs of words which are precisely related to the actions of the branches of the rational map $f$ on the Riemann sphere $\mathbb{C}_{\infty}$. There is a natural compactification of the space of paths in $\mathcal{W}$ which can be identified with the Julia set $J(f)$. We then show that the Julia set agrees with the Martin boundary. In many cases the Julia set turns out to be fractal, and it has been investigated by many authors from different viewpoints. There have been several approaches to introduce harmonic analysis on different fractal sets. We mention a few of them: first of all the construction of Brownian motion on the Sierpi'nski gasket due to Goldstein [29], Kusuoka [36] and Barlow/Perkins [6], secondly the identification of the Sierpi'nski gasket as a Martin boundary of a Markov chain (with an intention to establish harmonic analysis on the gasket) due to Denker and Sato ([16], [17]), thirdly the geometric construction by Kigami ([33], [34]). This continu- 
ing interest to develop harmonic analysis on fractal sets motivated our research besides its value for the boundary theory of Markov chains. The representation of the Julia set as the Martin boundary of a certain random walk may well be considered as the first step towards another approach to introduce harmonic analysis on the Julia set.

Our basic idea is to identify the set of finite words over a fixed finite alphabet with the successive contracting pieces of a set containing the Julia set (and thus tending to the Julia set), and then to define a Markov chain on the above set as the state space. We give an explicit formula of the Martin kernel (see Theorem 55) and then identify the Julia set with the space of exits (see Dynkin [22]). In the sequel, the formula for the Martin kernel allows us to describe the Martin space explicitly. There we show one of our main results, that the Julia set is homeomorphic to the Martin boundary via a Lipschitz map (see Lemma 64, Lemma 65, Lemma 66, Lemma 67). As a corollary to the result we have also derived the representation theorem for harmonic functions of the Markov chain. This result also shows that the Julia set is the space of exits (see Theorem 68).

The identification of the Julia set $J(f)$ with the Martin boundary is obtained by using techniques from symbolic dynamics; more specifically, the one-sided shift space $\Sigma^{+}$is used to "code" the Julia set and the Martin boundary. This connection enables us to relate different thermodynamic quantities, such as entropy, pressure, measure of maximal entropy, Gibbs measure, and measure of equilibrium, to similar potential theoretic quantities such as capacity, harmonic measures on the Julia set with a suitable potential $\phi$.

In our case we have, by using Mañé [43], identified the measure of maximal entropy for the rational map $f$ on the Julia set $J$; it is nothing but the image measure of the $(\underbrace{1 / d, \cdots, 1 / d}_{d \text {-times }})$ Bernoulli measure 
(on $\Sigma^{+}$) under the mapping $\Phi: \Sigma^{+} \rightarrow J$, where $d$ is the degree of the rational map $f$ (see Corollary 62).

We have also proven that the harmonic measure $\mu_{1}$ (related to the excessive function 1) on the Julia set $J$ (in the sense of Dynkin [22]) is the image measure of a nonatomic, quasi-invariant, conservative measure $\nu$ on the one-sided shift space $\Sigma^{+}$(see Theorem 69, Lemma 70, Theorem 74 and Lemma 76). We have shown that this quasi-invariant measure $\nu$ is equivalent to $(1 / d, 1 / d, \cdots, 1 / d)$ Bernoulli measure (see Lemma 75). We have also shown that the measure $\nu$ is a Gibbs measure for a certain potential $\psi$ on $\Sigma^{+}$(see Theorem 83), which arises from the theory of thermodynamics and thus connects the two different theories. Corollary 62, Theorem 69 and Lemma 75 imply the equivalence of the three measures: the Gibbs measure $\nu,(1 / d, 1 / d, \cdots, 1 / d)$-Bernoulli measure and the image measure of the measure of maximal entropy under a certain homeomorphism $\Phi$ (see Corollary 84).

Moreover, by using the Ruelle-Perron-Frobenius theorem we have deduced that the measure $\gamma=h \nu$ is the unique $\sigma$-invariant probability measure, called equilibrium measure for the potential $\psi$, with the property that

$$
P(\psi)=h_{\gamma}(\sigma)+\int_{\Sigma^{+}} \psi d \gamma=0,
$$

where $h>0$ is the eigenfunction of the Ruelle operator $\mathcal{L}_{\psi}$ and $P(\psi)$ denotes the pressure of $\psi$ (see Corollary 85).

We have further found that the measure of equilibrium for the logarithmic potential in our case also has close ties with the classical harmonic measure: it is well known (see e.g. Ransford [50]) that for a domain $D \subset \mathbb{C}_{\infty}$, with a non-polar boundary $\partial D$, there exists a unique harmonic measure $\omega_{D}$ for $D$. It is also known that if the rational map $R$ is hyperbolic, then its Julia set $J(R)$ has zero area (see e.g. [12]). This means that, the Julia set of a hyperbolic rational map is measure- 
theoretically negligible. However, $J(R)$ still can be a non-polar (which roughly means "not negligible" in the potential theoretic sense) set having a positive capacity $C(J(R))$. In that case there exists a unique equilibrium measure for the logarithmic potential on $J(R)$ (see e.g. Ransford [50]).

For a compact non-polar subset $K$ of $\mathbb{C}$, the measure of equilibrium for the logarithmic potential coincides with this unique harmonic measure $\nu=\omega_{D}(\infty, \cdot)$, where $D$ is the component of $\mathbb{C}_{\infty} \backslash K$ containing $\infty$ (see e.g. Ransford [50]). As these results perfectly fit into our scenario, we have deduced that these two measures coincide in our case (see Theorem 90).

As our Julia set has special properties (totally disconnected, nonpolar), it is natural to expect that the logarithmic potential (for its equilibrium measure) at each of its points would be the same. This is indeed the case: we have proven that the logarithmic potential is a constant function (see Theorem 91).

Since the logarithmic potential on our non-polar Julia set $p_{\nu}(\zeta)=$ $I(\nu)$, where $I(\nu)$ denotes the total energy for the equilibrium measure $\nu$, at each point $\zeta \in J$, it turns out that the Fatou domain $D=\mathbb{C}_{\infty} \backslash J$ is regular (see e.g. Theorem 4.2.4 in Ransford [50]). Consequently, we have a unique solution for the Dirichlet problem: that is, for a continuous function $\phi: \partial D \rightarrow \mathbb{R}$, there exists a unique harmonic function $h$ on D such that $\lim _{z \rightarrow \zeta} h(z)=\phi(\zeta)$ for all $\zeta \in \partial D$ (see Theorem 92). 


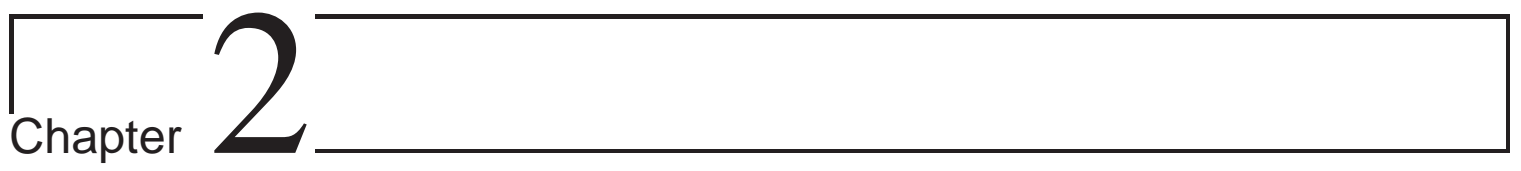

\section{Preliminaries}

In this chapter we simply state basic results from complex dynamical systems, ergodic theory, potential theory and fractal geometry which are needed in the sequel.

\subsection{Rational Map and its Julia set}

A rational map $f: \mathbb{C}_{\infty} \rightarrow \mathbb{C}_{\infty}$ is a holomorphic dynamical system on the Riemann sphere $\mathbb{C}_{\infty}=\mathbb{C} \cup\{\infty\}$. Any such map can be written as a quotient

$$
f(z)=\frac{P(z)}{Q(z)}=\frac{a_{0} z^{d}+\cdots+a_{d}}{b_{0} z^{d}+\cdots+b_{d}}
$$

of two relative prime polynomials $P$ and $Q$. The degree of $f$ can be defined topologically or algebraically; it is the number of pre-images of a typical point $z$, as well as the maximum of the degrees of $P$ and $Q$.

It is a crucial fact that when $f$ is a rational function of positive degree $d$, then $f$ is a $d$-fold map of $\mathbb{C}_{\infty}$ onto itself: that is, for any $w \in \mathbb{C}_{\infty}$ the equation $f(z)=w$ has precisely $d$ solutions in $z$ (counting multiplicities).

We say that two rational maps $R$ and $S$ are conjugate if and only if there is some Möbius map $g$ with $S=g \circ R \circ g^{-1}$.

An important property of conjugacy is that it respects iteration: 
that is, if $S=g \circ R \circ g^{-1}$ then $S^{n}=g \circ R^{n} \circ g^{-1}$. This means that we can transfer a problem concerning $R$ to a (possibly simpler) problem concerning a conjugate $S$ of $R$ and then attempt to solve this in terms of $S$. Yet another obvious property of conjugacy is that it respects fixed points, explicitly, if $S=g \circ R \circ g^{-1}$, then $S$ fixes $g(z)$ if and only if $R$ fixes $z$.

The transition probabilities of the Markov chain defined on the word space $\mathcal{W}$ (see $\S 3.1$ ) and the map $\Phi$ which establishes the homeomorphism between the shift space $\Sigma^{+}$and the Julia set $J$ are given in terms of inverse branches of a hyperbolic rational map. We now introduce all these terminologies and results.

A point $z$ is a critical point of a rational map $R$ if $R$ fails to be injective in any neighborhood of $z$. A value $w$ is a critical value for $R$ if it is the image of some critical point; that is, if $w=R(z)$ for some critical point $z$. If $R$ is of degree $d$ and $w$ is not a critical value, then $R^{-1}\{w\}$ consists of precisely $d$ distinct points, say $z_{1}, \cdots, z_{d}$. As none of the $z_{j}$ are critical points, there are neighborhoods $N$ of $w$, and $N_{1}, \cdots, N_{d}$ of $z_{1}, \cdots, z_{d}$ respectively, with $R$ acting as a bijection from each $N_{j}$ onto $N$. It follows that for each $j$, the restriction $R_{j}$ of the map $R$ to $N_{j}$ has an inverse

$$
R_{j}^{-1}: N \rightarrow N_{j}
$$

and we call these the branches of $R^{-1}$ at $w$.

Given a rational map $f: \mathbb{C}_{\infty} \rightarrow \mathbb{C}_{\infty}$ of degree $\geq 2$ on the Riemann sphere $\mathbb{C}_{\infty}$ the Fatou set, $F(f)$, is defined as follows

$$
\begin{gathered}
F(f)=\left\{z \in \mathbb{C}_{\infty} \mid \exists U \subset \mathbb{C}_{\infty} \text { an open neighborhood of } z\right. \text { so that } \\
\left.\left\{\left.f^{n}\right|_{U}\right\}_{n \geq 0} \text { is normal }\right\}
\end{gathered}
$$

The Julia set, $J(f)$, is then defined as the complement of the Fatou set, i.e. $J(f)=\mathbb{C}_{\infty} \backslash F(f)$. It is well known that $J(f)$ is non-empty, perfect, and fully invariant, i.e. $f(J(f))=J(f)=f^{-1}(J(f))$. Also note that by definition $J(f)$ is closed and hence compact, as $\mathbb{C}_{\infty}$ is compact.

We consider hyperbolic rational maps in the thesis. The dynamics of hyperbolic rational maps are best behaved and well understood. Now 
we give the definition of a hyperbolic rational map and state a theorem which characterizes such maps.

Definition 1 The postcritical set $P(f)$ is the closure of the forward orbits of the critical points of $f$ :

$$
P(f)=\bigcup_{n>0, f^{\prime}(c)=0} f^{n}(c)
$$

The postcritical set plays a crucial role with respect to the attractors of $f$. We now introduce the property of hyperbolicity.

Theorem 2 Let $f$ be a rational map of degree $d \geq 2$. The following conditions are equivalent:

1. All critical points of $f$ tend to attracting cycles under iteration.

2. The map $f$ is expanding on its Julia set. That is, there exists a conformal metric $\rho$ on the sphere such that $\left|f^{\prime}(z)\right|_{\rho}>1$ for all $z$ in the Julia set $J(f)$.

3. The postcritical set and the Julia set are disjoint:

$$
P(f) \cap J(f)=\emptyset .
$$

Proof: We refer to McMullen [40].

Definition 3 A rational map $f$ satisfying any (and hence all) of the above three conditions is said to be hyperbolic.

Now we introduce the notion of exceptional points as they will play an important role in establishing one of our results on the equivalent measures on the Julia set.

For any $z \in \mathbb{C}_{\infty}$, the backward orbit of $z$ for a rational map $R$, is the set

$$
\begin{aligned}
O^{-}(z) & =\left\{w: \text { for some } n \geq 0, R^{n}(w)=z\right\} \\
& =\cup_{n \geq 0} R^{-n}\{z\},
\end{aligned}
$$

and we call the points in $O^{-}(z)$ the predecessors of $z$. The following theorem characterizes the exceptional points. 
Theorem 4 The backward orbit $O^{-}(z)$ of $z$ is finite if and only if $z$ is exceptional.

Proof: We refer to Beardon [7].

The set of exceptional points for a rational map $R$ is denoted by $E(R)$. The following theorem justifies the terminology by showing that such points are indeed exceptional. The theorem will be used to prove one of our results on equivalent measures.

Theorem 5 A rational map $R$ of degree at least two has at most two exceptional points. If $E(R)=\{\zeta\}$, then $R$ is conjugate to a polynomial with $\zeta$ corresponding to $\infty$. If $E(R)=\left\{\zeta_{1}, \zeta_{2}\right\}$, where $\zeta_{1} \neq \zeta_{2}$, then $R$ is conjugate to some map $z \mapsto z^{d}$, where $\zeta_{1}$ and $\zeta_{2}$ correspond to 0 and $\infty$.

Proof: We refer to Beardon [7].

Corollary 6 If deg $R \geq 2$, then the exceptional points of $R$ lie in the Fatou set $F(R)$.

Proof: We refer to Beardon [7].

\subsection{Self-similar Sets}

In this section we simply give the definition of self-similar sets and selfsimilar structures and state a few resuts which will be used to deduce a result in our case. The details on self-similar sets can be found in Kigami [35].

First we state a theorem that ensures uniqueness and existence of self-similar sets.

Theorem 7 Let $(X, d)$ be a complete metric space. If $f_{i}: X \rightarrow X$ are contractions with respect to the metric $d$ for $i=1,2, \cdots, N$, then there exists a unique non-empty compact subset $K$ of $X$ that satisfies

$$
K=f_{1}(K) \cup f_{2}(K) \cup \cdots \cup f_{N}(K) .
$$

$K$ is called the self-similar set with respect to $\left\{f_{1}, f_{2}, \cdots, f_{N}\right\}$. 
Proof: We refer to Kigami [35].

It is shown in Kigami [35] that the word space $\mathcal{W}$ (which consists of words built from the alphabet $\mathcal{A}=\{1,2 \cdots, N\}$ ) and the one-sided shift space $\Sigma^{+}$are self-similar sets with respect to a set of suitable contraction maps.

The notion of self-similar structure gives a topological description of self-similar sets.

Definition 8 Let $K$ be a compact metrizable topological space and let $S$ be a finite set. Also, let $F_{i}$ be a continuous injection from $K$ to itself for any $i \in S$. Then $\left(K, S,\left\{F_{i}\right\}_{i \in S}\right)$ is called a self-similar structure if there exists a continuous surjection $\pi: \Sigma^{+} \rightarrow K$ such that $F_{i} \circ \pi=\pi \circ \sigma_{i}$ for every $i \in S$, where $\Sigma^{+}$is the one-sided shift space, and $\sigma_{i}: \Sigma^{+} \rightarrow \Sigma^{+}$ is defined by

$$
\sigma_{i}\left(w_{1} w_{2} w_{3} \cdots\right)=i w_{1} w_{2} w_{3} \cdots
$$

for each $w_{1} w_{2} w_{3} \cdots \in \Sigma^{+}$.

Remark: It follows from the above definition that

$$
K=\bigcup_{i \in S} F_{i}(K)
$$

Obviously, if $K$ is the self-similar set with respect to injective contractions $\left\{f_{1}, f_{2}, f_{3}, \cdots, f_{N}\right\}$, then $\left(K,\{1,2, \cdots, N\},\left\{f_{i}\right\}_{i=1}^{N}\right)$ is a selfsimilar structure.

Proposition 9 If $\left(K, S,\left\{F_{i}\right\}_{i \in S}\right)$ is a self-similar structure, then the corresponding surjection $\pi$ is unique. In fact,

$$
\{\pi(\omega)\}=\bigcap_{m \geq 0} F_{\omega_{1} \omega_{2} \cdots \omega_{m}}(K)
$$

for any $\omega=\omega_{1} \omega_{2} \cdots \in \Sigma^{+}$.

Proof: We refer to Kigami [35]. 
Definition 10 Let $\mathcal{L}=\left(K, S,\left\{F_{i}\right\}_{i \in S}\right)$ be a self-similar structure. We define $C_{\mathcal{L}, K}=\cup_{i, j \in S, i \neq j}\left(F_{i}(K) \cap F_{j}(K)\right), C_{\mathcal{L}}=\pi^{-1}\left(C_{\mathcal{L}, K}\right)$ and $\mathcal{P}_{\mathcal{L}}=$ $\cup_{n \geq 1} \sigma^{n}\left(\mathcal{C}_{\mathcal{L}}\right) . C_{\mathcal{L}}$ is called the critical set of $\mathcal{L}$ and $P_{\mathcal{L}}$ is called the post critical set of $\mathcal{L}$. We also define $V_{0}(\mathcal{L})=\pi\left(\mathcal{P}_{\mathcal{L}}\right)$.

For ease of notation, we use $\mathcal{C}, \mathcal{P}$ and $V_{0}$ instead of $\mathcal{C}_{\mathcal{L}}, \mathcal{P}_{\mathcal{L}}$ and $V_{0}(\mathcal{L})$ as long as it can not cause any confusion.

The critical set and the post critical set play an important role in determining the topological structure of a self-similar set. For example, if $\mathcal{C}=\emptyset$, (and hence $\mathcal{P}, V_{0}$ are all empty sets), then $K$ is homeomorphic to the (topological) Cantor set $\Sigma^{+}$.

Proposition 11 Let $\mathcal{L}=\left(K, S,\left\{F_{i}\right\}_{i \in S}\right)$ be a self-similar structure. Then

(1) $\pi^{-1}\left(V_{0}\right)=\mathcal{P}$

(2) If $\Sigma_{\boldsymbol{w}} \cap \Sigma_{\boldsymbol{v}}=\emptyset$ for $\boldsymbol{w}, \boldsymbol{w} \in \mathcal{W}$, then $K_{\boldsymbol{w}} \cap K_{\boldsymbol{v}}=F_{\boldsymbol{w}}\left(V_{0}\right) \cap F_{\boldsymbol{v}}\left(V_{0}\right)$, where $K_{\boldsymbol{w}}=F_{\boldsymbol{w}}(K)$.

(3) $\mathcal{C}=\emptyset$ if and only if $\pi$ is injective.

Proof: We refer to Kigami [35].

Definition 12 Let $\mathcal{L}=\left(K, S,\left\{F_{i}\right\}_{i \in S}\right)$ be a self-similar structure. $\mathcal{L}$ is said to be post critically finite, or p.c.f. for short, if and only if the post critical set $\mathcal{P}_{\mathcal{L}}$ is a finite set.

\subsection{Results from Potential Theory}

In this section we briefly mention few results from potential theory which will be useful to prove a few of our results.

Definition 13 Let $X$ be a topological space. We say that a function $u: X \rightarrow[-\infty, \infty)$ is upper semicontinuous if the set $\{x \in X: u(x)<$ $\alpha\}$ is open in $X$ for each $\alpha \in \mathbb{R}$. Also $v: X \rightarrow(-\infty, \infty]$ is lower semicontinuous if $-v$ is upper semicontinuous. 
Definition 14 Let $U$ be an open subset of $\mathbb{C}$. A function $u: U \rightarrow$ $[-\infty, \infty)$ is called subharmonic if it is upper semicontinuous and satisfies the local submean inequality, i.e. given $w \in U$, there exists $\rho>0$ such that

$$
u(w) \leq \frac{1}{2 \pi} \int_{0}^{2 \pi} u\left(w+r e^{i t}\right) d t(0 \leq r<\rho)
$$

Also $v: U \rightarrow(-\infty, \infty]$ is superharmonic if $-v$ is subharmonic.

Potentials provide an important source of examples of superharmonic functions.

Definition 15 Let $\mu$ be a finite Borel measure on $\mathbb{C}$ with compact support. Its logarithmic potential is the function $p_{\mu}: \mathbb{C} \rightarrow(-\infty, \infty]$ defined by

$$
p_{\mu}(z)=\int \log \frac{1}{|z-w|} d \mu(w) \quad(z \in \mathbb{C}) .
$$

Theorem $16 p_{\mu}$ is superharmonic on $\mathbb{C}$, and harmonic on $\mathbb{C} \backslash(\operatorname{supp} \mu)$. Also

$$
p_{\mu}(z)=-\mu(\mathbb{C}) \log |z|+\mathcal{O}\left(|z|^{-1}\right) \text { as } z \rightarrow \infty .
$$

Proof: We refer to Ransford [50].

Polar sets play the role of negligible sets in potential theory, much as sets of measure zero do in measure theory. To define them, we first need to introduce the notion of energy.

Definition 17 Let $\mu$ be a finite Borel measure on $\mathbb{C}$ with compact support. Its energy $I(\mu)$ is given by

$$
I(\mu):=\iint \log \frac{1}{|z-w|} d \mu(z) d \mu(w)=\int p_{\mu}(z) d \mu(z) .
$$

To explain this terminology, think of $\mu$ as being a charge distribution on $\mathbb{C}$. Then $p_{\mu}(z)$ represents the potential energy at $z$ due to $\mu$, and so the total energy of $\mu$ is just $\int p_{\mu}(z) d \mu(z)$, in other words $I(\mu)$. It is possible that $I(\mu)=+\infty$. Indeed some sets only support measures of infinite energy. These are important enough to deserve having a name. 
Definition 18 (a) A subset $E$ of $\mathbb{C}$ is called polar if $I(\mu)=+\infty$ for every finite Borel measure $\mu \neq 0$ for which supp $\mu$ is a compact subset of $E$.

(b) A property is said to hold quasi-everywhere (q.e.) on a subset $S$ of $\mathbb{C}$ if it holds everywhere on $S \backslash E$, for some Borel polar set $E$.

Clearly singleton sets are polar. Also every subset of a polar set is polar. In the other direction, if a set is non-polar, then it contains a compact subset which is non-polar (namely supp $\mu$, for some measure $\mu$ with $I(\mu)<+\infty)$.

It is easy to see that a measure of finite energy can have no atoms. More generally, measures of finite energy do not charge polar sets.

Theorem 19 Let $\mu$ be a finite Borel measure on $\mathbb{C}$ with compact support, and suppose that $I(\mu)<+\infty$. Then $\mu(E)=0$ for every Borel polar set $E$.

Proof: We refer to Ransford [50].

Corollary 20 Every Borel polar set has Lebesgue measure zero.

Proof: We refer to Ransford [50].

Thus, quasi-everywhere implies almost everywhere.

Definition 21 The logarithmic capacity of a subset $E$ of $\mathbb{C}$ is given by

$$
c(E):=e^{-v(E)}
$$

where $v(E) \equiv \inf \left\{I(\mu): \mu \in \mathcal{P}^{c}(E)\right\}, \mathcal{P}^{c}(E)$ is the collection of all Borel probability measures $\mu$ on $\mathbb{C}$ whose support is a compact subset of $E$.

Here it is understood that $e^{-\infty}=0$, so that $c(E)=0$ precisely when $E$ is polar. A set with positive capacity is called non-polar.

The next theorem lists a few properties of logarithmic capacity. 
Theorem 22 (a) If $E_{1} \subset E_{2}$, then $c\left(E_{1}\right) \leq c\left(E_{2}\right.$.)

(b) If $E \subset \mathbb{C}$, then $c(E)=\sup \{c(K):$ compact $K \subset E\}$.

(c) If $K$ is a compact subset of $\mathbb{C}$ then $c(K)=c\left(\partial_{e} K\right)$, where $\partial_{e} K$ denotes the exterior boundary of $K$.

Proof: We refer to Ransford [50].

Lemma 23 Let $T(z)=a z+b$ be a linear transformation and $E_{1}=$ $T(E)$. Then $c\left(E_{1}\right)=|a| c(E)$.

Proof: We refer to Tsuji [54].

Lemma 24 If $E$ is a continuum, then $c(E)>0$.

Proof: We refer to Tsuji [54].

Theorem 25 If $K$ is a compact set with positive capacity, then there is a unique probability measure $\mu$ with support contained in $K$ such that

$$
I(\mu)=v(K):=\inf \{I(\nu): \nu \in \mathcal{P}(K)\},
$$

where $\mathcal{P}(K)$ is the collection of all Borel probability measure on $K$.

Proof: We refer to Ransford [50].

Definition 26 If $K$ is a compact set and $\mu \in \mathcal{P}(K)$ such that $I(\mu)=$ $v(K)$, then $\mu$ is called an equilibrium measure for $K$. The corresponding logarithmic potential $p_{\mu}$ is called a conductor or equilibrium potential of $K$.

Physical intuition would tend to suggest that if $\mu$ is an equilibrium measure for $K$ then $p_{\mu}$ should be constant on $K$ ( for otherwise charge would flow from one part of $K$ to another, disturbing the equilibrium). The idea is confirmed by the next theorem:

Theorem 27 (Frostman's Theorem) If $K$ is a compact set and $\mu$ is an equilibrium measure, then $p_{\mu} \leq v(K)$ on $\mathbb{C}$ and $p_{\mu}=v(K)$ everywhere on $K$ except for an $F_{\sigma}$ set with capacity zero. 
Proof: We refer to Ransford [50].

Now we state a few results which are related to the Dirichlet problem and harmonic measures for a proper subdomain $D$ of $\mathbb{C}_{\infty}$.

Definition 28 Let $D$ be a proper subdomain of $\mathbb{C}_{\infty}$ and let $\phi: \partial D \rightarrow \mathbb{R}$ be a bounded function. The associated Perron function $H_{D} \phi: D \rightarrow \mathbb{R}$ is defined by

$$
H_{D} \phi=\sup _{u \in \mathcal{U}} u
$$

where $\mathcal{U}$ denotes the family of all subharmonic functions $u$ on $D$ such that $\lim \sup _{z \rightarrow \zeta} u(z) \leq \phi(\zeta)$ for each $\zeta \in \partial D$.

The next theorem simply states that $H_{D} \phi$ is always a bounded harmonic function.

Theorem 29 Let $D$ be a proper subdomain of $\mathbb{C}_{\infty}$, let $\phi: \partial D \rightarrow \mathbb{R}$ be a bounded function. Then $H_{D} \phi$ is harmonic on $D$, and

$$
\sup _{D}\left|H_{D} \phi\right| \leq \sup _{\partial D}|\phi|
$$

Proof: We refer to Ransford [50].

Definition 30 Let $D$ be a proper subdomain of $\mathbb{C}_{\infty}$, and let $\zeta_{0} \in \partial D$. $A$ barrier at $\zeta_{0}$ is a subharmonic function $b$ defined on $D \cap N$, where $N$ is an open neighborhood of $\zeta_{0}$, satisfying

$$
b<0 \text { on } D \cap N \text { and } \lim _{z \rightarrow \zeta_{0}} b(z)=0 .
$$

A boundary point at which a barrier exists is called regular, otherwise it is irregular. If every $\zeta \in \partial D$ is regular, then $D$ is called a regular domain.

Theorem 31 (Solution of the Dirichlet Problem) Let $D$ be a regular domain, and let $\phi: \partial D \rightarrow \mathbb{R}$ be a continuous function. Then there exists a unique harmonic function $h$ on $D$ such that

$$
\lim _{z \rightarrow \zeta} h(z)=\phi(\zeta)
$$

for all $\zeta \in \partial D$. 
Proof: We refer to Ransford [50].

We will use the following theorem to prove that our domain is a regular one.

Theorem 32 Let $D$ be a proper subdomain of $\mathbb{C}_{\infty}$, and let $\zeta_{0} \in \partial D$. Set $K=\mathbb{C}_{\infty} \backslash D$. Then the following assertions are equivalent:

(a) $\zeta_{0}$ is a regular boundary point of $D$;

(b) $K$ is non-thin at $\zeta_{0}$.

If also $\infty \in D$, then these are equivalent to:

(c) $K$ is non-polar, and $p_{\nu}\left(\zeta_{0}\right)=I(\nu)$, where $\nu$ is the equilibrium measure for $K$.

Proof: We refer to Ransford [50].

Remark: As we will be using only the equivalence of $(a)$ and $(c)$, we skip the notion of thinness.

The following theorem will be used to relate the harmonic measure to the equilibrium measure on the Julia set:

Theorem 33 Let $K$ be a compact non-polar subset of $\mathbb{C}$. Then its equilibrium measure $\mu$ is given by

$$
\mu=\omega_{D}(\infty, \cdot)
$$

where $D$ is the component of $\mathbb{C}_{\infty} \backslash K$ containing $\infty$.

Proof: We refer to Ransford [50].

\subsection{Shift space and Ruelle Operator}

Definition 34 Let $A$ be a $d \times d$ matrix of zeros and ones $(d \geq 2)$, where the $(i, j)$-th entry is zero precisely when it is a prohibited word of length 2. We define

$$
\Sigma:=\left\{x=\left(x_{n}\right)_{n=-\infty}^{n=\infty}: x_{n} \in\{1, \cdots, d\}, n \in \mathbb{Z}, A\left(x_{n}, x_{n+1}\right)=1\right\} .
$$

If $\{1, \cdots, d\}$ is given the discrete topology then $\Sigma$ is compact with the corresponding Tychonov product topology. The shift $\sigma$ is defined by 
$\sigma(x)=y$, where $y_{n}=x_{n+1}$ i.e. all sequences are shifted one place to the left. The pair $(\Sigma, \sigma)$ is called a shift of finite type (or topological Markov chain).

The matrix $A$ is called irreducible if for each pair $(i, j), 1 \leq i, j \leq d$, there exists $n \geq 1$ such that $A^{n}(i, j)>0$, where $A^{n}$ is an $n$-fold product of $A$ with itself. Under this condition we define the period $p$ of $A$ to be the highest common factor of $\left\{n: A^{n}(i, i)>0,1 \leq i \leq d\right\}$. When $p=1, A$ is called aperiodic.

To every (two-sided) shift of finite type we can associate a (one sided) shift of finite type $\left(\Sigma^{+}, \sigma^{+}\right)$:

$$
\Sigma^{+}=\left\{x=\left(x_{n}\right)_{n=0}^{n=\infty}: x_{n} \in\{1, \cdots, d\}, n \geq 0, A\left(x_{n}, x_{n+1}\right)=1\right\}
$$

and $\sigma^{+} x=y, y_{n}=x_{n+1}, n \geq 0$, i.e. all sequences are shifted one place to the left, with the first term being deleted. As before, $\Sigma^{+}$is compact with the corresponding Tychonov product topology.

An elementary, but important, difference is that whereas the twosided shift is a homeomorphism, the one-sided shift is not invertible (but merely a local homeomorphism). There is a natural continuous surjection $\pi: \Sigma \rightarrow \Sigma^{+}$with $\pi(x)=y, y_{n}=x_{n}, n \geq 0$, i.e. one deletes the terms $x_{n}, n<0$. This surjection clearly satisfies the identity $\pi \circ \sigma=\sigma^{+} \circ \pi$.

To simplify our notation as far as possible we shall write $\sigma$ for both $\sigma$ and $\sigma^{+}$. As we will use the one-sided shift space to "encode" our Julia set, we study $\Sigma^{+}$. The situation is very similar for $\Sigma$.

Given $0<\theta<1$ we can define a metric $d_{\theta}$ on $\Sigma^{+}$by $d_{\theta}(x, y)=\theta^{N}$, where $N$ is the largest integer such that $x_{i}=y_{i}, 0 \leq i<N$. For a continuous function $f: \Sigma^{+} \rightarrow \mathbb{C}$ and $n \geq 0$ we define

$$
\begin{gathered}
\operatorname{var}_{n} f=\sup \left\{|f(x)-f(y)|: x_{i}=y_{i}, 0 \leq i<n\right\}, \\
\qquad|f|_{\theta}=\sup \left\{\frac{\operatorname{var}_{n} f}{\theta^{n}} \mid n \geq 0\right\} \\
\text { and }|f|_{\infty}=\sup \left\{|f(x)|: x \in \Sigma^{+}\right\} .
\end{gathered}
$$


It is easy to see that

$$
|f(x)-f(y)| \leq C d_{\theta}(x, y) \Longleftrightarrow \operatorname{var}_{n} f \leq C \theta^{n}, n=0,1, \ldots
$$

for some constant $C>0$.

We let

$$
\begin{aligned}
\mathcal{F}_{\theta}^{+} & =\mathcal{F}_{\theta}^{+}\left(\Sigma^{+}\right) \\
& =\left\{f: f \text { continuous, } \operatorname{var}_{n} f \leq C \theta^{n}, n=0,1, \ldots, \text { for some } C>0\right\} .
\end{aligned}
$$

Thus $\mathcal{F}_{\theta}^{+}$is the space of Lipschitz functions with respect to the metric $d_{\theta}$.

For $f \in \mathcal{F}_{\theta}^{+}$we define the Ruelle operator $\mathcal{L}_{f}: \mathcal{F}_{\theta}^{+} \rightarrow \mathcal{F}_{\theta}^{+}$(or more generally, $\mathcal{L}_{f}: C\left(\Sigma^{+}\right) \rightarrow C\left(\Sigma^{+}\right), C\left(\Sigma^{+}\right)$being the space of continuous functions on $\Sigma^{+}$), by

$$
\left(\mathcal{L}_{f} w\right)(x)=\sum_{\sigma y=x} e^{f(y)} w(y) .
$$

It is easy to see that $\mathcal{L}_{f}$ is a bounded linear operator. When $f$ is real and $\mathcal{L}_{f} 1=1$ we say that $f$ or $\mathcal{L}_{f}$ is normalised.

We need the following result which is called the Ruelle-Perron-Frobenius Theorem:

Theorem 35 Let $f \in \mathcal{F}_{\theta}^{+}$be real valued and suppose $A$ is aperiodic. Then we have:

(i) There is a simple maximal positive eigenvalue $\beta$ of $\mathcal{L}_{f}: C\left(\Sigma^{+}\right) \rightarrow$ $C\left(\Sigma^{+}\right)$with a corresponding strictly positive eigenfunction $h \in \mathcal{F}_{\theta}^{+}$.

(ii) The remainder of the spectrum of $\mathcal{L}_{f}: \mathcal{F}_{\theta}^{+} \rightarrow \mathcal{F}_{\theta}^{+}$(excluding $\beta>0)$ is contained in a disc of radius strictly smaller than $\beta$.

(iii) There is a unique probability measure $\mu$ such that $\mathcal{L}_{f}^{*} \mu=\beta \mu$, i.e. $\int \mathcal{L}_{f} v d \mu=\beta \int v d \mu$ for all $v \in C\left(\Sigma^{+}\right)$.

(iv) $\frac{1}{\beta^{n}} \mathcal{L}_{f}^{n} v \rightarrow h \int v d \mu$ uniformly for all $v \in C\left(\Sigma^{+}\right)$where $h$ is as above and $\int h d \mu=1$.

Proof: We refer to Parry [47]. 


\subsection{Entropy Pressure and Gibbs Measure}

Entropy and pressure are important quantities giving useful information on the dynamics of a system. In this section we briefly mention a few results on these two quantities together with their connections to the Gibbs measure.

If $\mathcal{C}=\left\{C_{1}, \cdots, C_{k}\right\}$ is a partition of a measure space $(X, \mathcal{B}, \mu)$ (i.e., the $C_{i}$ 's are pairwise disjoint and $X=\cup_{i=1}^{k} C i$ ), one defines the entropy

$$
H_{\mu}(\mathcal{C})=\sum_{i=1}^{k}\left(-\mu\left(C_{i}\right) \log \mu\left(C_{i}\right)\right) .
$$

If $\mathcal{D}$ is another (finite) partition of that space,

$$
\mathcal{C} \vee \mathcal{D}=\left\{C_{i} \cap D_{j}: C_{i} \in \mathcal{C}, D_{j} \in \mathcal{D}\right\}
$$

Lemma 36 If $\mathcal{D}$ is a (finite) partition of $(X, \mathcal{B}, \mu)$ and $T$ is an automorphism of $(X, \mathcal{B}, \mu)$, then

$$
h_{\mu}(T, \mathcal{D})=\lim _{m \rightarrow \infty} \frac{1}{m} H_{\mu}\left(\mathcal{D} \vee T^{-1} \mathcal{D} \vee \cdots \vee T^{-m+1} \mathcal{D}\right)
$$

exists.

Proof: We refer to Bowen [10].

Notation: The set of Borel probability measures on $\Sigma^{+}$which are invariant (i.e. $\left.\mu\left(\sigma^{-1} E\right)=\mu(E)\right)$ is denoted by $M_{\sigma}\left(\Sigma^{+}\right)$.

Definition 37 Let $\mu \in M_{\sigma}\left(\Sigma^{+}\right)$and $\mathcal{U}=\left\{U_{1}, U_{2}, \cdots, U_{d}\right\}$ where $U_{i}=$ $\left\{x \in \Sigma^{+}: x_{0}=i\right\}$. Then $s(\mu)=h_{\mu}(\sigma, \mathcal{U})$ is called the entropy of $\mu$.

Suppose now that $\phi \in C\left(\Sigma^{+}\right)$and that $a_{0} a_{1} \cdots a_{m-1}$ are integers between 1 and $n$ satisfying $A_{a_{k} a_{k+1}}=1$. Write

$$
\begin{aligned}
& \sup _{a_{0} a_{1} \cdots a_{m-1}} S_{m} \phi=\sup \left\{\sum_{k=0}^{m-1} \phi\left(\sigma^{k} x\right): x \in \Sigma^{+}, x_{i}=a_{i}, \text { for all } 0 \leq 1<m\right\} \\
& \text { and } Z_{m}(\phi)=\sum_{a_{0} a_{1} \cdots a_{m-1}} \exp \left(\sup _{a_{0} a_{1} \cdots a_{m-1}} S_{m} \phi\right) \text {. }
\end{aligned}
$$


Lemma 38 For $\phi \in C\left(\Sigma^{+}\right)$, the following limit

$$
P(\phi)=\lim _{m \rightarrow \infty} \frac{1}{m} \log Z_{m}(\phi)
$$

exists (called the topological pressure of $\phi$ ).

Proof: We refer to Bowen [10].

Lemma 39 The topological pressure of $f$ is $P(f)=\log \beta$.

Proof: In order to show that $P(f)=\log \beta$, we simply apply (iv) of the Ruelle-Perron-Frobenius Theorem 35 to the function $v$ which is constantly equal to 1 .

Remark: Note that since

$$
\sup _{a_{0} a_{1} \cdots a_{m-1}} S_{m} \phi \geq \sum_{k=0}^{m-1} \phi\left(\sigma^{k}(x) \geq-m\|\phi\|,\right.
$$

we have

$$
Z_{m}(\phi) \geq \exp \left(\sup _{a_{0} a_{1} \cdots a_{m-1}} S_{m} \phi\right) \geq \exp (-m\|\phi\|) ;
$$

which in turn implies

$$
\log Z_{m}(\phi) \geq-m\|\phi\|
$$

As $\phi$ is continuous on the compact space $\Sigma^{+}, \phi$ is bounded. Hence the pressure $P(\phi)$ is finite.

We need the following two theorems to prove one of our results.

Theorem 40 Entropy is a conjugacy invariant and hence an isomorphism invariant.

Proof: We refer to Walters [55].

Theorem 41 Let $T_{i}: X_{i} \rightarrow X_{i}(i=1,2)$ be a continuous transformation of a compact metrizable space and suppose $T_{i}$ has a unique measure, 
$\mu_{i}$, with maximal entropy. Suppose $h_{\mu_{1}}\left(T_{1}\right)=h_{\mu_{2}}\left(T_{2}\right)$. If $\phi: X_{1} \rightarrow X_{2}$ is a bimeasurable bijection with $\phi \circ T_{1}=T_{2} \circ \phi$ then $\mu_{1} \circ \phi^{-1}=\mu_{2}$ [and so $\phi$ is an isomorphism between the measure-preserving transformations $T_{i}$ on $\left.\left(X_{i}, \mathcal{B}\left(X_{i}\right), \mu_{i}\right)\right]$.

Proof: We refer to Walters [55].

Usually Gibbs measures are studied as invariant probability measures in the literature. However, they can be non-invariant; the following definition of the Gibbs measure appears in Parry [47].

Definition 42 A probability measure $m$ on $\Sigma^{+}$is called a Gibbs measure if there exists $\psi \in C\left(\Sigma^{+}\right)$such that

$$
A \leq \frac{m\left(\left[x_{0} x_{1} \cdots x_{n}\right]\right)}{\exp \left(C \cdot n+S_{n} \psi(x)\right)} \leq B
$$

for $n \geq 0$ and fixed constants $A, B>0$, and $C \in \mathbb{R}$.

Note that we do not necessarily require that $m$ should be $\sigma$-invariant. The following proposition describes those measures which have the Gibbs property for a certain class of functions.

Proposition 43 When $f \in \mathcal{F}_{\theta}^{+}$is real and normalized we have the following inequality:

$$
\exp \left(-|f|_{\theta} \theta^{n}\right) \leq \frac{m\left[x_{0} \cdots x_{n}\right] \exp (-f(x))}{m\left[x_{1} \cdots x_{n}\right]} \leq \exp \left(|f|_{\theta} \theta^{n}\right)
$$

where $|f|_{\theta}=\sup \left\{\frac{\operatorname{var}_{n} f}{\theta^{n}} \mid n \geq 0\right\}$, and $\mathcal{L}_{f}^{*} m=m$ as in RuellePerron-Frobenius' Theorem.

Proof: We refer to Parry [47].

Corollary $44 \mathrm{~m}$ is a Gibbs measure with pressure $P(f)=0$. 
Proof: We refer to Parry [47].

Remark: The above two results can easily be adjusted to deal with the case where $f \in \mathcal{F}_{\theta}^{+}$and where we no longer necessarily assume that $\mathcal{L}_{f}$ is normalized: we simply apply the above proposition to $g=$ $f-\log h \circ \sigma+\log h-\log \beta$, where $h, \beta$ are the positive eigenfunction and eigenvalue guaranteed by the Ruelle-Perron-Frobenius Theorem. 


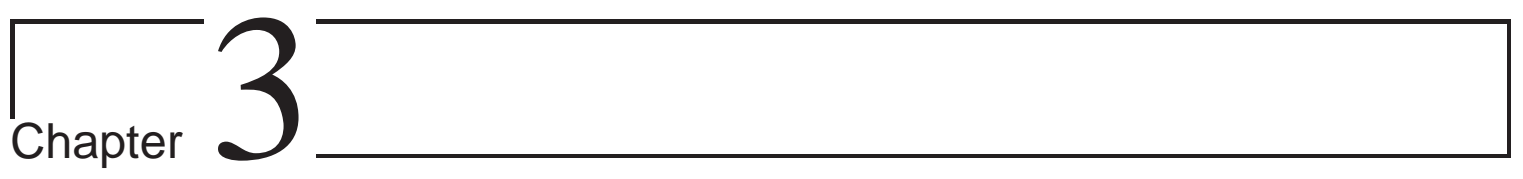

\section{Julia Set as a Martin Boundary}

In this chapter we prove that a totally disconnected Julia set of a hyperbolic rational map can be identified as a Martin boundary of a certain Markov chain on the word space $\mathcal{W}$. The definition of our Markov chain and the notion of the Martin kernel and boundary appear in $\S 3.1$ and $\S 3.2$ respectively. We have calculated an explicit formula for the Martin kernel associated with the Markov chain in $\S 3.3$. The notion of shift space appears in $\S 3.4$ where we have proven that the Julia set is homeomorphic to the shift space via a Lipschitz map. The last $\S 3.5$ contains the proof of the identification of the Julia set as a Martin boundary of a certain Markov chain.

\subsection{Markov Chain on the Word Space}

Definition 45 Let $\mathcal{A}=\{1,2, \cdots, d\}$ be the alphabet of $d$ letters ( $d \geq$ 2) and

$$
\mathcal{W}_{+}=\left\{w_{1} w_{2} w_{3} \cdots w_{n}: w_{i} \in \mathcal{A}, n \geq 1\right\}
$$

be the space of finite words. The empty word is denoted by the formal symbol $\emptyset$, which satisfies $\boldsymbol{w} \emptyset=\emptyset \boldsymbol{w}=\boldsymbol{w}$ for any element $\boldsymbol{w} \in \mathcal{W}_{+}$. Define the word space $\mathcal{W}=\mathcal{W}_{+} \cup\{\emptyset\}$.

For a fixed alphabet $\mathcal{A}=\{1,2, \cdots, d\}$, a Markov chain with state space $\mathcal{W}$ will be defined by the transition probabilities $p(\cdot, \cdot)$ on $\mathcal{W} \times \mathcal{W}$ 
as given in Definition 46. The existence of such a Markov chain is wellknown (see Chung [13] or Meyn [45]):

Notation: For $\boldsymbol{u} \in \mathcal{W}_{+}$, let $\boldsymbol{u}[n], 1 \leq n \leq d(\boldsymbol{u})$, denote the word that is obtained from the word $\boldsymbol{u}$ by deleting $n$ consecutive letters from the right. We also put $\boldsymbol{u}(d(\boldsymbol{u}))=\emptyset$. For example, if $\boldsymbol{u}=u_{1} u_{2} \cdots u_{l}$, then $\boldsymbol{u}[1]=u_{1} u_{2} \cdots u_{l-1}, \boldsymbol{u}[2]=u_{1} u_{2} \cdots u_{l-2}$ and $\boldsymbol{u}[l]=\emptyset$. We further put

$$
f_{\boldsymbol{u}}(z)=f_{u_{1}} \circ f_{u_{2}} \circ \cdots \circ f_{u_{l}}(z)
$$

where $f_{i}(i=1,2, \cdots, d)$ is an inverse branch of the rational map $f$ with degree $d \geq 2$, and $z, z \notin J$, is a (non-exceptional) point in a sufficiently small neighborhood $U_{\epsilon}$ of the Julia set $J(f)$.

Definition 46 The one-step transition probability on $\mathcal{W}$ for a continuous map $\phi: U_{\epsilon} \rightarrow \mathbb{R}$, a constant $c \in \mathbb{R}$, and $\boldsymbol{x}=x_{1} x_{2} \cdots x_{N}, \boldsymbol{y}=$ $y_{1} y_{2} \cdots y_{N^{\prime}} \in \mathcal{W}$, is defined by

$$
p(\boldsymbol{x}, \boldsymbol{y}):=p(1 ; \boldsymbol{x}, \boldsymbol{y}):=\frac{e^{\left[\phi\left(f_{\boldsymbol{y}}(z)\right)-c\right]} \chi_{\left\{\boldsymbol{w}: \boldsymbol{w}=x_{1} x_{2} \cdots x_{N} l, l=1,2, \cdots, d\right\}}(\boldsymbol{y})}{\sum_{\left\{\boldsymbol{w}: \boldsymbol{w}=x_{1} x_{2} \cdots x_{N} l, l=1,2, \cdots, d\right\}} e^{\left[\phi\left(f_{\boldsymbol{w}}(z)\right)-c\right]}}
$$

From Definition 46 we immediately have

$$
p(1 ; \boldsymbol{x}, \boldsymbol{y})=\frac{\sum_{l=1}^{d} e^{\left[\phi\left(f_{\boldsymbol{y}}(z)\right)-c\right]} \chi_{\left\{\boldsymbol{w}: w=x_{1} x_{2} \cdots x_{N} l\right\}}(\boldsymbol{y})}{\sum_{l=1}^{d} e^{\left[\phi\left(f_{\boldsymbol{x} l}(z)\right)-c\right]}}
$$

For $d=2$ we have the following tree diagram (Figure 3.1) which shows the transitions on the word space $\mathcal{W}$. Each node in the diagram represents a word and an arrow connecting two words depicts the onestep transition.

Lemma 47 If $p(1 ; \boldsymbol{x}, \boldsymbol{y})>0$ for $\boldsymbol{x} \in \mathcal{W}, \boldsymbol{y} \in \mathcal{W}_{+}$and $\boldsymbol{y}=\boldsymbol{u} a$ for some $\boldsymbol{u} \in \mathcal{W}, a \in \mathcal{A}$, then $\boldsymbol{x}=\boldsymbol{u}$.

Proof: The proof is obvious: If $\boldsymbol{x} \neq \boldsymbol{u}$, then from Definition 46 we immediately have $p(1 ; \boldsymbol{x}, \boldsymbol{y})=0$. 


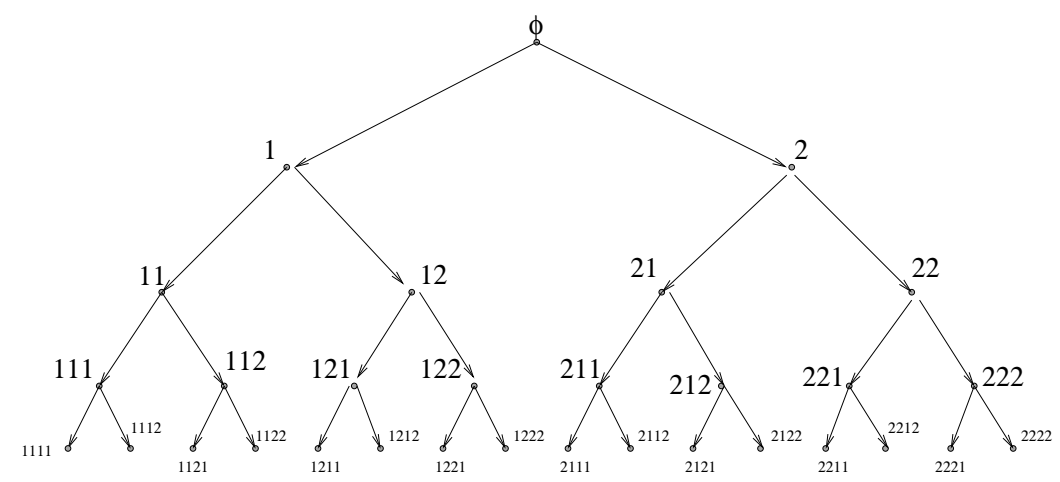

Figure 3.1: Transitions on the word space

The $n$-step transition probabilities are defined recursively by

$$
p(n ; \boldsymbol{v}, \boldsymbol{w})=\sum_{\boldsymbol{u} \in \mathcal{W}} p(n-1 ; \boldsymbol{v}, \boldsymbol{u}) p(1 ; \boldsymbol{u}, \boldsymbol{w}), \quad \boldsymbol{v}, \boldsymbol{w} \in \mathcal{W}, n \geq 1,
$$

where $p(0 ; \boldsymbol{v}, \boldsymbol{w})=\delta_{\boldsymbol{v}}(\boldsymbol{w})$ with $\delta_{\boldsymbol{v}}$ denoting the Dirac function at $\boldsymbol{v}$. Clearly we have $p(n ; \boldsymbol{v}, \boldsymbol{w})>0$ only if $d(\boldsymbol{v}, \boldsymbol{w})=n$, hence the Green function $g: \mathcal{W} \times \mathcal{W} \rightarrow \mathbb{R}_{+}$is well defined by

$$
g(\boldsymbol{v}, \boldsymbol{w})=\sum_{n=0}^{\infty} p(n ; \boldsymbol{v}, \boldsymbol{w})=p(d(\boldsymbol{v}, \boldsymbol{w}) ; \boldsymbol{v}, \boldsymbol{w}), \quad \boldsymbol{v}, \boldsymbol{w} \in \mathcal{W}
$$

A word $\boldsymbol{v}$ is called an ancestor of a word $\boldsymbol{w}$ and $\boldsymbol{w}$ is called a successor of $\boldsymbol{v}$ if $g(\boldsymbol{v}, \boldsymbol{w})>0$. This is denoted by $\boldsymbol{v} \ll \boldsymbol{w}($ or $\boldsymbol{w} \gg \boldsymbol{v})$. In particular, if $\boldsymbol{v} \ll \boldsymbol{w}$ and $d(\boldsymbol{w})-d(\boldsymbol{v})=k$, then $\boldsymbol{v}$ is called a $k$-ancestor of $\boldsymbol{w}$. The set of all $k$-ancestor of $\boldsymbol{w}$ is denoted by $A n c_{k}[\boldsymbol{w}]$. A 1-ancestor of $\boldsymbol{w}$ is also called a parent of $\boldsymbol{w}$. By definition, every $\boldsymbol{w} \in \mathcal{W}$ has at most one parent.

Lemma 48 For any $\boldsymbol{v}, \boldsymbol{w} \in \mathcal{W}$ and $1 \leq k \leq d(\boldsymbol{v}, \boldsymbol{w})$ we have

$$
g(\boldsymbol{v}, \boldsymbol{w})=\sum_{d(\boldsymbol{v}, \boldsymbol{u})=k, \boldsymbol{v} \ll \boldsymbol{u} \ll \boldsymbol{w}} g(\boldsymbol{v}, \boldsymbol{u}) g(\boldsymbol{u}, \boldsymbol{w})
$$


Proof: We have

$$
\begin{aligned}
g(\boldsymbol{v}, \boldsymbol{w}) & =p(d(\boldsymbol{v}, \boldsymbol{w}) ; \boldsymbol{v}, \boldsymbol{w}) \\
& =\sum_{\boldsymbol{u} \in \mathcal{W}} p(k ; \boldsymbol{v}, \boldsymbol{u}) p(d(\boldsymbol{v}, \boldsymbol{w})-k ; \boldsymbol{u}, \boldsymbol{w}) \\
& =\sum_{d(\boldsymbol{v}, \boldsymbol{u})=k, \boldsymbol{v} \ll \boldsymbol{u} \ll \boldsymbol{w}} p(k ; \boldsymbol{v}, \boldsymbol{u}) p(d(\boldsymbol{v}, \boldsymbol{w})-k ; \boldsymbol{u}, \boldsymbol{w}) \\
& =\sum_{d(\boldsymbol{v}, \boldsymbol{u})=k, \boldsymbol{v} \ll \boldsymbol{u} \ll \boldsymbol{w}} p(k ; \boldsymbol{v}, \boldsymbol{u}) p(d(\boldsymbol{w})-d(\boldsymbol{u}) ; \boldsymbol{u}, \boldsymbol{w}) \\
& =\sum_{d(\boldsymbol{v}, \boldsymbol{u})=k, \boldsymbol{v} \ll \boldsymbol{u} \ll \boldsymbol{w}} g(\boldsymbol{v}, \boldsymbol{u}) g(\boldsymbol{u}, \boldsymbol{w})
\end{aligned}
$$

Lemma 49 For any $\boldsymbol{y} \in \mathcal{W}_{+}$, we have

$$
g(\emptyset, \boldsymbol{y})=\frac{e^{\left[\phi\left(f_{\boldsymbol{y}}(z)\right)+\phi\left(f_{\boldsymbol{y}[1]}(z)\right)+\phi\left(f_{\boldsymbol{y}[2]}(z)\right)+\cdots+\phi\left(f_{\boldsymbol{y}[(\boldsymbol{y})-1]}(z)\right)-d(\boldsymbol{y}) c\right]}}{\Theta(\boldsymbol{y}[1]) \Theta(\boldsymbol{y}[2]) \Theta(\boldsymbol{y}[3]) \cdots \Theta(\boldsymbol{y}[d(\boldsymbol{y})-1]) \Theta(\emptyset)}
$$

where for $\boldsymbol{v} \in \mathcal{W}$

$$
\Theta(\boldsymbol{v})=\sum_{l=1}^{d} e^{\left[\phi\left(f_{\boldsymbol{v} l}(z)\right)-c\right]}
$$

Proof: We prove the lemma by induction on $d(\boldsymbol{y})$. By using Definition 46 , it is easy to see that the result holds for $d(\boldsymbol{y})=1$. Assume that the lemma is true for $d(\boldsymbol{y})=k \geq 1$ and let $\boldsymbol{y} \in \mathcal{W}, d(\boldsymbol{y})=k+1$. Then $\boldsymbol{y}$ can be written as $\boldsymbol{y}=\boldsymbol{u} l_{k+1}^{\prime}$ for some $\boldsymbol{u}=l_{1}^{\prime} l_{2}^{\prime} \cdots l_{k}^{\prime} \in \mathcal{W}, l_{k+1}^{\prime} \in \mathcal{A}$, 
and $d(\boldsymbol{u})=k$. Now by using equation (3.1) we obtain

$$
\begin{aligned}
& g(\emptyset, \boldsymbol{y})=\sum_{d\left(\emptyset, \boldsymbol{u}^{\prime}\right)=k, \emptyset \ll \boldsymbol{u}^{\prime} \ll \boldsymbol{y}} g\left(\emptyset, \boldsymbol{u}^{\prime}\right) g\left(\boldsymbol{u}^{\prime}, \boldsymbol{y}\right) \\
= & g(\emptyset, \boldsymbol{u}) g(\boldsymbol{u}, \boldsymbol{y}) \\
= & g(\emptyset, \boldsymbol{y}[1]) g(\boldsymbol{y}[1], \boldsymbol{y}) \\
= & g(\emptyset, \boldsymbol{y}[1]) p(1 ; \boldsymbol{y}[1], \boldsymbol{y}) \\
= & \frac{e^{\left[\phi\left(f_{\boldsymbol{y}[1]}(z)\right)+\phi\left(f_{\boldsymbol{y}[2]}(z)\right)+\cdots+\phi\left(f_{\boldsymbol{y}[1][d(\boldsymbol{y}[1])-1]}(z)\right)-d(\boldsymbol{y}[1]) c\right]}}{\Theta(\boldsymbol{y}[2]) \Theta(\boldsymbol{y}[3]) \cdots \Theta(\boldsymbol{y}[1][d(\boldsymbol{y}[1])-1]) \Theta(\emptyset)} \times \frac{e^{\left[\phi\left(f_{\boldsymbol{y}}(z)\right)-c\right]}}{\Theta(\boldsymbol{y}[1])} \\
= & \frac{e^{\left[\phi\left(f_{\boldsymbol{y}}(z)\right)+\phi\left(f_{\boldsymbol{y}[1]}(z)\right)+\phi\left(f_{\boldsymbol{y}[2]}(z)\right)+\cdots+\phi\left(f_{\boldsymbol{y}[1][d(\boldsymbol{y}[1])-1]}(z)\right)-(d(\boldsymbol{y}[1])+1) c\right]}}{\Theta(\boldsymbol{y}[1]) \Theta(\boldsymbol{y}[2]) \Theta(\boldsymbol{y}[3]) \cdots \Theta(\boldsymbol{y}[1][d(\boldsymbol{y}[1])-1]) \Theta(\emptyset)} \\
= & \frac{e^{\left[\phi\left(f_{\boldsymbol{y}}(z)\right)+\phi\left(f_{\boldsymbol{y}[1]}(z)\right)+\phi\left(f_{\boldsymbol{y}[2]}(z)\right)+\cdots+\phi\left(f_{\boldsymbol{y}[d(\boldsymbol{y})-1]}(z)\right)-d(\boldsymbol{y}) c\right]}}{\Theta(\boldsymbol{y}[1]) \Theta(\boldsymbol{y}[2]) \Theta(\boldsymbol{y}[3]) \cdots \Theta(\boldsymbol{y}[d(\boldsymbol{y})-1]) \Theta(\emptyset)}
\end{aligned}
$$

since $\boldsymbol{y}[1][d(\boldsymbol{y}[1])-1]=\boldsymbol{y}[d(\boldsymbol{y})-1]$.

\subsection{Martin Kernel and Martin Boundary}

We shall give a brief description of the theory of Martin boundaries adapted to our purpose. The details can be found in Dynkin [22].

Let $p(1 ; \boldsymbol{v}, \boldsymbol{w})$ with $\boldsymbol{v}, \boldsymbol{w} \in \mathcal{W}$ be the transition probability and let $\left\{X_{n}\right\}$ be the associated Markov chain. The Markov operator $P$ is defined by

$$
(P f)(\boldsymbol{v})=\sum_{\boldsymbol{w} \in \mathcal{W}} p(1 ; \boldsymbol{v}, \boldsymbol{w}) f(w), \boldsymbol{v} \in \mathcal{W}
$$

for a non-negative function $f$ on $\mathcal{W}$.

A non-negative function $f: \mathcal{W} \rightarrow \mathbb{R}$ is called $P$-excessive if

$$
(P f)(\boldsymbol{v}) \leq f(\boldsymbol{v}), \quad \boldsymbol{v} \in \mathcal{W}
$$

and P-harmonic if

$$
(P f)(\boldsymbol{v})=f(\boldsymbol{v}), \quad \boldsymbol{v} \in \mathcal{W} .
$$


The Martin kernel is defined by

$$
k(\boldsymbol{v}, \boldsymbol{w})=\frac{g(\boldsymbol{v}, \boldsymbol{w})}{g(\emptyset, \boldsymbol{w})}, \quad \boldsymbol{v}, \boldsymbol{w} \in \mathcal{W} .
$$

By Lemma $49, g(\emptyset, \cdot)$ is positive, so the Martin kernel is well defined for $g$.

Now we define a metric $\rho$ on $\mathcal{W}$ as in the following lemma:

Lemma 50 The map $\rho: \mathcal{W} \times \mathcal{W} \rightarrow \mathbb{R}$ defined by

$$
\rho(\boldsymbol{v}, \boldsymbol{w}):=\left|2^{-d(\boldsymbol{v})}-2^{-d(\boldsymbol{w})}\right|+\sum_{\boldsymbol{u} \in \mathcal{W}} a(\boldsymbol{u}) \frac{|k(\boldsymbol{u}, \boldsymbol{w})-k(\boldsymbol{u}, \boldsymbol{v})|}{1+|k(\boldsymbol{u}, \boldsymbol{w})-k(\boldsymbol{u}, \boldsymbol{v})|}
$$

where $\{a(\boldsymbol{u}) ; \boldsymbol{u} \in \mathcal{W}\}$ is some fixed sequence of strictly positive numbers such that $\sum_{u \in \mathcal{W}} a(\boldsymbol{u})=1$, is a metric on $\mathcal{W}$.

Proof: Clearly $\rho(\boldsymbol{v}, \boldsymbol{w}) \geq 0, \rho(\boldsymbol{v}, \boldsymbol{v})=0$ and $\rho(\boldsymbol{v}, \boldsymbol{w})=\rho(\boldsymbol{w}, \boldsymbol{v})$ for all $\boldsymbol{v}, \boldsymbol{w} \in \mathcal{W}$. The triangle inequality follows from the following inequality:

$$
\frac{|\alpha+\beta|}{1+|\alpha+\beta|} \leq \frac{|\alpha|}{1+|\alpha|}+\frac{|\beta|}{1+|\beta|}, \quad \alpha, \beta \in \mathbb{R}
$$

Now we show that $\rho(\boldsymbol{v}, \boldsymbol{w})=0$ implies that $\boldsymbol{v}=\boldsymbol{w}$. Then from (3.2) we have $d(\boldsymbol{v})=d(\boldsymbol{w})$ and $k(\boldsymbol{u}, \boldsymbol{w})=k(\boldsymbol{u}, \boldsymbol{v})$ for all $\boldsymbol{u} \in \mathcal{W}$. Letting $\boldsymbol{u}=\boldsymbol{w}$ in the last equality and using the fact that $k(\boldsymbol{w}, \boldsymbol{w}) \neq 0$ we obtain $\boldsymbol{v}=\boldsymbol{w}$.

In this metric $\rho$, it is easy to see that a sequence $\left\{\boldsymbol{w}_{n}\right\}$ in $\mathcal{W}$ is Cauchy if and only if $\boldsymbol{w}_{n}$ is eventually constant, say equal $\boldsymbol{w} \in \mathcal{W}$, or

$$
d\left(\boldsymbol{w}_{n}\right) \rightarrow \infty \text { and } \lim _{n} k\left(\boldsymbol{v}, \boldsymbol{w}_{n}\right) \text { exists for any } \boldsymbol{v} \in \mathcal{W}
$$

Two Cauchy sequences $\left\{\boldsymbol{w}_{n}\right\}$ and $\left\{\boldsymbol{u}_{n}\right\}$ are called equivalent if

$$
\lim _{n \rightarrow \infty} \rho\left(\boldsymbol{u}_{n}, \boldsymbol{w}_{n}\right)=0 .
$$


Let $\overline{\mathcal{W}}$ be the collection of all equivalence classes of Cauchy sequences in $\mathcal{W}$. Then $\overline{\mathcal{W}}$ is the $\rho$-completion of $\mathcal{W}$ and is called the Martin space. This is a compact metric space(see Lemma 54 below) with the extension of $\rho$, and $\mathcal{W}$ is an open dense subset of $\overline{\mathcal{W}}$. The boundary

$$
M=\partial \overline{\mathcal{W}}=\overline{\mathcal{W}} \backslash \mathcal{W}
$$

is called the Martin boundary. Clearly, it is also a compact metric space.

Lemma 51 For every fixed $\boldsymbol{v} \in \mathcal{W}$, the map $\boldsymbol{w} \mapsto k(\boldsymbol{v}, \boldsymbol{w})$ is uniformly continuous in the metric $\rho$.

Proof: Let $\epsilon>0$ be given. We need to show that there exists $\delta=\delta(\epsilon)>0$ such that

$$
\rho\left(\boldsymbol{w}_{1}, \boldsymbol{w}_{2}\right)<\delta, \quad \boldsymbol{w}_{1}, \boldsymbol{w}_{2} \in \mathcal{W} \Rightarrow\left|k\left(\boldsymbol{v}, \boldsymbol{w}_{1}\right)-k\left(\boldsymbol{v}, \boldsymbol{w}_{2}\right)\right|<\epsilon .
$$

Now as

$$
\begin{aligned}
& \rho\left(\boldsymbol{w}_{1}, \boldsymbol{w}_{2}\right)=\left|2^{-d\left(\boldsymbol{w}_{1}\right)}-2^{-d\left(\boldsymbol{w}_{2}\right)}\right|+\sum_{\boldsymbol{u} \in \mathcal{W}} a(\boldsymbol{u}) \frac{\left|k\left(\boldsymbol{u}, \boldsymbol{w}_{2}\right)-k\left(\boldsymbol{u}, \boldsymbol{w}_{1}\right)\right|}{1+\left|k\left(\boldsymbol{u}, \boldsymbol{w}_{2}\right)-k\left(\boldsymbol{u}, \boldsymbol{w}_{1}\right)\right|} \\
&=\left|2^{-d\left(\boldsymbol{w}_{1}\right)}-2^{-d\left(\boldsymbol{w}_{2}\right)}\right|+a(\boldsymbol{v}) \frac{\left|k\left(\boldsymbol{v}, \boldsymbol{w}_{2}\right)-k\left(\boldsymbol{v}, \boldsymbol{w}_{1}\right)\right|}{1+\left|k\left(\boldsymbol{v}, \boldsymbol{w}_{2}\right)-k\left(\boldsymbol{v}, \boldsymbol{w}_{1}\right)\right|} \\
&+\sum_{\boldsymbol{u} \in \mathcal{W}, \boldsymbol{u} \neq \boldsymbol{v}} a(\boldsymbol{u}) \frac{\left|k\left(\boldsymbol{u}, \boldsymbol{w}_{2}\right)-k\left(\boldsymbol{u}, \boldsymbol{w}_{1}\right)\right|}{1+\left|k\left(\boldsymbol{u}, \boldsymbol{w}_{2}\right)-k\left(\boldsymbol{u}, \boldsymbol{w}_{1}\right)\right|},
\end{aligned}
$$

and if we choose $\delta=\frac{\epsilon}{\epsilon+1} a(\boldsymbol{v})$, it follows

$$
\begin{aligned}
\rho\left(\boldsymbol{w}_{1}, \boldsymbol{w}_{2}\right)<\delta & \Rightarrow a(\boldsymbol{v}) \frac{\left|k\left(\boldsymbol{v}, \boldsymbol{w}_{2}\right)-k\left(\boldsymbol{v}, \boldsymbol{w}_{1}\right)\right|}{1+\left|k\left(\boldsymbol{v}, \boldsymbol{w}_{2}\right)-k\left(\boldsymbol{v}, \boldsymbol{w}_{1}\right)\right|}<\delta \\
& \Rightarrow\left|k\left(\boldsymbol{v}, \boldsymbol{w}_{2}\right)-k\left(\boldsymbol{v}, \boldsymbol{w}_{1}\right)\right|<\epsilon
\end{aligned}
$$

Lemma 52 Let $(X, d)$ be a metric space and let $A$ be a non-empty subset of $X$. Then the mapping $x \mapsto d(x, A)$ is uniformly continuous. 
Proof: Let $\epsilon>0$. Choose $\delta \leq \epsilon$. Then,

$$
d(x, y)<\delta \Rightarrow|d(x, A)-d(y, A)| \leq d(x, y)<\delta \leq \epsilon .
$$

Thus, $\quad d(x, y)<\delta \Rightarrow|d(x, A)-d(y, A)| \leq d(x, y)<\epsilon$.

Lemma 53 Let $\left(X, d_{1}\right)$ be a metric space and $A$ be a dense subset of $X$. Let $\left(Y, d_{2}\right)$ be a complete metric space and $f: A \rightarrow Y$ be a uniformly continuous function. Then $f$ can be extended uniquely to a uniformly continuous function $g: X \rightarrow Y$.

Proof: We refer to any standard text on metric spaces.

As a consequence of the above three lemmas, for every fixed $\boldsymbol{v} \in \mathcal{W}$ the functions $\boldsymbol{w} \rightarrow k(\boldsymbol{v}, \boldsymbol{w})$ and $\boldsymbol{w} \rightarrow \rho(\boldsymbol{v}, \boldsymbol{w})$ have extensions to uniformly continuous functions on $\overline{\mathcal{W}}$. The extensions are also denoted by $k(\boldsymbol{v}, \zeta)$, $\rho(\boldsymbol{v}, \zeta), \zeta \in \overline{\mathcal{W}}$.

Lemma $\mathbf{5 4} \overline{\mathcal{W}}$ is a compact metric space.

Proof: Let $\left\{w_{n}\right\}_{n \in \mathbb{N}}$ be any arbitrary but fixed sequence in $\overline{\mathcal{W}}$. We note that the sequence $\left\{k\left(w, w_{n}\right)\right\}_{n \in \mathbb{N}}$ is a bounded sequence of real numbers for fixed $w \in \mathcal{W}$. So we can choose a subsequence $\left\{w_{N(n)}\right\}_{n \in \mathbb{N}} \subset$ $\overline{\mathcal{W}}$ such that $\left\{k\left(w, w_{N(n)}\right)\right\}_{n \in \mathbb{N}}$ is convergent. Then by using the property of the metric $\rho$ in (3.2) we can conclude that $\left\{w_{N(n)}\right\}_{n \in \mathbb{N}}$ is Cauchy in $\overline{\mathcal{W}}$. Since $\overline{\mathcal{W}}$ is complete, $\left\{w_{N(n)}\right\}_{n \in \mathbb{N}}$ converges in $\overline{\mathcal{W}}$. Thus $\overline{\mathcal{W}}$ is sequentially compact which is equivalent to compactness for metric spaces.

\subsection{Determination of the Martin Kernel}

In this section we compute the $n$-step transition probability $p(n ; \boldsymbol{x}, \boldsymbol{y})$ with a view to calculating the Martin kernel. For simplicity, we set

$$
S_{1}=\{\boldsymbol{u} \in \mathcal{W} \mid \boldsymbol{u}=\boldsymbol{x} l, l=1,2, \cdots, d\}
$$


and

$$
S_{2}=\{\boldsymbol{u} \in \mathcal{W} \mid \boldsymbol{u} \neq \boldsymbol{x} l, l=1,2, \cdots, d\}
$$

where $\boldsymbol{x}=x_{1} x_{2} \cdots x_{N}$. Then

$$
\begin{aligned}
& p(2 ; \boldsymbol{x}, \boldsymbol{y})=\sum_{\boldsymbol{u} \in \mathcal{W}} p(1 ; \boldsymbol{x}, \boldsymbol{u}) p(1 ; \boldsymbol{u}, \boldsymbol{y}) \\
& =\left(\sum_{\boldsymbol{u} \in S_{1}}+\sum_{\boldsymbol{u} \in S_{2}}\right) p(1 ; \boldsymbol{x}, \boldsymbol{u}) p(1 ; \boldsymbol{u}, \boldsymbol{y}) \\
& =\left(\sum_{\boldsymbol{u} \in S_{1}}+\sum_{\boldsymbol{u} \in S_{2}}\right)\left[\frac{\sum_{l_{1}=1}^{d} e^{\left[\phi\left(f_{\boldsymbol{u}}(z)\right)-c\right]} \chi_{\left\{\boldsymbol{w}: \boldsymbol{w}=\boldsymbol{x} l_{1}\right\}}(\boldsymbol{u})}{\sum_{l_{1}=1}^{d} e^{\left[\phi\left(f_{\boldsymbol{x} l_{1}}(z)\right)-c\right]}} \times\right. \\
& \left.\frac{\sum_{i_{1}=1}^{d} e^{\left[\phi\left(f_{\boldsymbol{y}}(z)\right)-c\right]} \chi_{\left\{\boldsymbol{w}: \boldsymbol{w}=\boldsymbol{u} i_{1}\right\}}(\boldsymbol{y})}{\sum_{i_{1}=1}^{d} e^{\left[\phi\left(f_{\boldsymbol{u} i_{1}}(z)\right)-c\right]}}\right] \\
& =\sum_{\boldsymbol{u} \in S_{1}}\left[\frac{\sum_{l_{1}=1}^{d} e^{\left[\phi\left(f_{\boldsymbol{u}}(z)\right)-c\right]} \chi_{\left\{\boldsymbol{w}: \boldsymbol{w}=\boldsymbol{x} l_{1}\right\}}(\boldsymbol{u})}{\sum_{l_{1}=1}^{d} e^{\left[\phi\left(f_{\boldsymbol{x} l_{1}}(z)\right)-c\right]}} \times\right. \\
& \left.\frac{\sum_{i_{1}=1}^{d} e^{\left[\phi\left(f_{\boldsymbol{y}}(z)\right)-c\right]} \chi_{\left\{\boldsymbol{w}: \boldsymbol{w}=\boldsymbol{u} i_{1}\right\}}(\boldsymbol{y})}{\sum_{i_{1}=1}^{d} e^{\left[\phi\left(f_{\boldsymbol{u} i_{1}}(z)\right)-c\right]}}\right] \\
& =\frac{e^{\left[\phi\left(f_{\boldsymbol{x} 1}(z)\right)-c\right]}}{\sum_{l_{1}=1}^{d} e^{\left[\phi\left(f_{\boldsymbol{x} l_{1}}(z)\right)-c\right]}} \times \frac{\sum_{i_{1}=1}^{d} e^{\left[\phi\left(f_{\boldsymbol{y}}(z)\right)-c\right]} \chi_{\left\{\boldsymbol{w}: \boldsymbol{w}=\boldsymbol{x} 1 i_{1}\right\}}(\boldsymbol{y})}{\sum_{i_{1}=1}^{d} e^{\left[\phi\left(f_{\boldsymbol{x} 1 i_{1}}(z)\right)-c\right]}} \\
& +\frac{e^{\left[\phi\left(f_{\boldsymbol{x} 2}(z)\right)-c\right]}}{\sum_{l_{1}=1}^{d} e^{\left[\phi\left(f_{\boldsymbol{x} l_{1}}(z)\right)-c\right]}} \times \frac{\sum_{i_{1}=1}^{d} e^{\left[\phi\left(f_{\boldsymbol{y}}(z)\right)-c\right]} \chi_{\left\{\boldsymbol{w}: \boldsymbol{w}=\boldsymbol{x} 2 i_{1}\right\}}(\boldsymbol{y})}{\sum_{i_{1}=1}^{d} e^{\left[\phi\left(f_{\boldsymbol{x} 2 i_{1}}(z)\right)-c\right]}} \\
& \vdots \\
& +\frac{e^{\left[\phi\left(f_{\boldsymbol{x} d}(z)\right)-c\right]}}{\sum_{l_{1}=1}^{d} e^{\left[\phi\left(f_{\boldsymbol{x} l_{1}}(z)\right)-c\right]}} \times \frac{\sum_{i_{1}=1}^{d} e^{\left[\phi\left(f_{\boldsymbol{y}}(z)\right)-c\right]} \chi_{\left\{\boldsymbol{w}: \boldsymbol{w}=\boldsymbol{x} d i_{1}\right\}}(\boldsymbol{y})}{\sum_{i_{1}=1}^{d} e^{\left[\phi\left(f_{\boldsymbol{x} d i_{1}}(z)\right)-c\right]}}
\end{aligned}
$$




$$
\begin{aligned}
& =\frac{1}{\sum_{l_{1}=1}^{d} e^{\left[\phi\left(f_{\boldsymbol{x} l_{1}}(z)\right)-c\right]}} \sum_{i_{2}=1}^{d}\left[\frac{e^{\left[\phi\left(f_{\boldsymbol{x} i_{2}}(z)\right)-c\right]}}{\sum_{l_{2}=1}^{d} e^{\left[\phi\left(f_{\boldsymbol{x} i_{2} l_{2}}(z)\right)-c\right]}} \times\right. \\
& \left.\sum_{i_{1}=1}^{d} e^{\left[\phi\left(f_{\boldsymbol{y}}(z)\right)-c\right]} \chi_{\left\{\boldsymbol{w}: \boldsymbol{w}=\boldsymbol{x} i_{2} i_{1}\right\}}(\boldsymbol{y})\right] \\
& =\sum_{i_{1}, i_{2}=1}^{d}\left[\frac{e^{\left[\phi\left(f_{\boldsymbol{y}}(z)\right)+\phi\left(f_{\boldsymbol{x} i_{2}}(z)\right)-2 c\right]} \chi_{\left\{\boldsymbol{w}: \boldsymbol{w}=\boldsymbol{x} i_{2} i_{1}\right\}}(\boldsymbol{y})}{\sum_{l_{1}=1}^{d} e^{\left[\phi\left(f_{\boldsymbol{x} l_{1}}(z)\right)-c\right]} \sum_{l_{2}=1}^{d} e^{\left[\phi\left(f_{\boldsymbol{x} i_{2} l_{2}}(z)\right)-c\right]}}\right]
\end{aligned}
$$

We claim that $p(n ; \boldsymbol{x}, \boldsymbol{y})$

$$
\begin{array}{r}
\sum_{i_{1}, i_{2}, \cdots, i_{n}=1}^{d} \frac{e^{\left[\phi\left(f_{\boldsymbol{y}}(z)\right)+\phi\left(f_{\boldsymbol{x} i_{n} i_{n-1} \cdots i_{2}}(z)\right)+\cdots+\phi\left(f_{\boldsymbol{x} i_{n}}(z)\right)-n c\right]}}{\Theta(\boldsymbol{x}) \Theta\left(\boldsymbol{x} i_{n}\right) \Theta\left(\boldsymbol{x} i_{n} i_{n-1}\right) \cdots \Theta\left(\boldsymbol{x} i_{n} i_{n-1} \cdots i_{2}\right)} \times \\
\chi_{\left\{\boldsymbol{w}: \boldsymbol{w}=\boldsymbol{x} i_{n} i_{n-1} \cdots i_{2} i_{1}\right\}}(\boldsymbol{y})
\end{array}
$$

Clearly, the claim holds for $n=1,2$. Suppose it holds for $n$. We show that it also holds for $n+1$. Now

$$
\begin{aligned}
& p(n+1 ; \boldsymbol{x}, \boldsymbol{y})=\sum_{u \in \mathcal{W}} p(1 ; \boldsymbol{x}, \boldsymbol{u}) p(n ; \boldsymbol{u}, \boldsymbol{y}) \\
&= \sum_{\boldsymbol{u} \in \mathcal{W}}\left[\frac{\sum_{l_{1}=1}^{d} e^{\left[\phi\left(f_{\boldsymbol{u}}(z)\right)-c\right]} \chi_{\left\{\boldsymbol{w}: w=\boldsymbol{x} l_{1}\right\}}(\boldsymbol{u})}{\sum_{l_{1}=1}^{d} e^{\left[\phi\left(f_{\boldsymbol{x} l_{1}}(z)\right)-c\right]}} \times\right. \\
& \sum_{i_{1}, i_{2}, \cdots, i_{n}=1}^{d} \frac{e^{\left[\phi\left(f_{\boldsymbol{y}}(z)\right)+\phi\left(f_{\boldsymbol{u} i_{n} i_{n-1} \cdots i_{2}}(z)\right)+\cdots+\phi\left(f_{\boldsymbol{u} i_{n}}(z)\right)-n c\right]}}{\Theta(\boldsymbol{u}) \Theta\left(\boldsymbol{u} i_{n}\right) \Theta\left(\boldsymbol{u} i_{n} i_{n-1}\right) \cdots \Theta\left(\boldsymbol{u} i_{n} i_{n-1} \cdots i_{2}\right)} \times \\
&= \sum_{\boldsymbol{u} \in S_{1} \cup S_{2}}\left[\frac{\left.\sum_{\left\{\boldsymbol{w}: \boldsymbol{w}=\boldsymbol{u} i_{n} i_{n-1} \cdots i_{2} i_{1}\right\}}(\boldsymbol{y})\right]}{\sum_{l_{1}=1}^{d} e^{\left[\phi\left(f_{\boldsymbol{u}}(z)\right)-c\right]} \chi_{\left\{\boldsymbol{w}: w=\boldsymbol{x} l_{1}\right\}}(\boldsymbol{u})} \times\right. \\
& \sum_{i_{1}, i_{2}, \cdots, i_{n}=1}^{d} e^{\left[\phi\left(f_{\boldsymbol{x} l_{1}}(z)\right)-c\right]} \frac{e^{\left[\phi\left(f_{\boldsymbol{y}}(z)\right)+\phi\left(f_{\boldsymbol{u} i_{n} i_{n-1} \cdots i_{2}}(z)\right)+\cdots+\phi\left(f_{\boldsymbol{u} i_{n}}(z)\right)-n c\right]}}{\Theta(\boldsymbol{u}) \Theta\left(\boldsymbol{u} i_{n}\right) \Theta\left(\boldsymbol{u} i_{n} i_{n-1}\right) \cdots \Theta\left(\boldsymbol{u} i_{n} i_{n-1} \cdots i_{2}\right)} \times \\
&\left.\chi_{\left\{\boldsymbol{w}: \boldsymbol{w}=\boldsymbol{u} i_{n} i_{n-1} \cdots i_{2} i_{1}\right\}}(\boldsymbol{y})\right]
\end{aligned}
$$




$$
\begin{aligned}
& =\frac{e^{\left[\phi\left(f_{\boldsymbol{x} 1}(z)\right)-c\right]}}{\sum_{l_{1}=1}^{d} e^{\left[\phi\left(f_{x l_{1}}(z)\right)-c\right]}} \times \\
& \sum_{i_{1}, i_{2}, \cdots, i_{n}=1}^{d} \frac{e^{\left[\phi\left(f_{\boldsymbol{y}}(z)\right)+\phi\left(f_{\boldsymbol{x} 1 i_{n} i_{n-1} \cdots i_{2}}(z)\right)+\cdots+\phi\left(f_{\boldsymbol{x} 1 i_{n}}(z)\right)-n c\right]}}{\Theta(\boldsymbol{x} 1) \Theta\left(\boldsymbol{x} 1 i_{n}\right) \Theta\left(\boldsymbol{x} 1 i_{n} i_{n-1}\right) \cdots \Theta\left(\boldsymbol{x} 1 i_{n} i_{n-1} \cdots i_{2}\right)} \times \\
& \chi_{\left\{\boldsymbol{w}: \boldsymbol{w}=\boldsymbol{x} 1 i_{n} i_{n-1} \cdots i_{2} i_{1}\right\}}(\boldsymbol{y}) \\
& +\frac{e^{\left[\phi\left(f_{\boldsymbol{x} 2}(z)\right)-c\right]}}{\sum_{l_{1}=1}^{d} e^{\left[\phi\left(f_{x l_{1}}(z)\right)-c\right]}} \times \\
& \sum_{i_{1}, i_{2}, \cdots, i_{n}=1}^{d} \frac{e^{\left[\phi\left(f_{\boldsymbol{y}}(z)\right)+\phi\left(f_{\boldsymbol{x} 2 i_{n} i_{n-1} \cdots i_{2}}(z)\right)+\cdots+\phi\left(f_{\boldsymbol{x} 2 i_{n}}(z)\right)-n c\right]}}{\Theta(\boldsymbol{x} 2) \Theta\left(\boldsymbol{x} 2 i_{n}\right) \Theta\left(\boldsymbol{x} 2 i_{n} i_{n-1}\right) \cdots \Theta\left(\boldsymbol{x} 2 i_{n} i_{n-1} \cdots i_{2}\right)} \times \\
& \chi_{\left\{\boldsymbol{w}: \boldsymbol{w}=\boldsymbol{x} 2 i_{n} i_{n-1} \cdots i_{2} i_{1}\right\}}(\boldsymbol{y}) \\
& \vdots \\
& +\frac{e^{\left[\phi\left(f_{\boldsymbol{x} d}(z)\right)-c\right]}}{\sum_{l_{1}=1}^{d} e^{\left[\phi\left(f_{x l_{1}}(z)\right)-c\right]}} \times \\
& \sum_{i_{1}, i_{2}, \cdots, i_{n}=1}^{d} \frac{e^{\left[\phi\left(f_{\boldsymbol{y}}(z)\right)+\phi\left(f_{\boldsymbol{x} d i_{n} i_{n-1} \cdots i_{2}}(z)\right)+\cdots+\phi\left(f_{\boldsymbol{x} d i_{n}}(z)\right)-n c\right]}}{\Theta(\boldsymbol{x} d) \Theta\left(\boldsymbol{x} d i_{n}\right) \Theta\left(\boldsymbol{x} d i_{n} i_{n-1}\right) \cdots \Theta\left(\boldsymbol{x} d i_{n} i_{n-1} \cdots i_{2}\right)} \times \\
& \chi_{\left\{\boldsymbol{w}: \boldsymbol{w}=\boldsymbol{x} d i_{n} i_{n-1} \cdots i_{2} i_{1}\right\}}(\boldsymbol{y}) \\
& =\sum_{i_{n+1}=1}^{d} \frac{\left.\left.e^{\left[\phi \left(f_{\boldsymbol{x i}}+1\right.\right.}(z)\right)-c\right]}{\sum_{l_{1}=1}^{d} e^{\left[\phi\left(f_{\boldsymbol{x} l_{1}}(z)\right)-c\right]}} \times \\
& \sum_{i_{1}, i_{2}, \cdots, i_{n}=1}^{d} \frac{e^{\left[\phi\left(f_{\boldsymbol{y}}(z)\right)+\phi\left(f_{\boldsymbol{x} i_{n+1} i_{n} i_{n-1} \cdots i_{2}}(z)\right)+\cdots+\phi\left(f_{\boldsymbol{x} i_{n+1} i_{n}}(z)\right)-n c\right]}}{\Theta\left(\boldsymbol{x} i_{n+1}\right) \Theta\left(\boldsymbol{x} i_{n+1} i_{n}\right) \cdots \Theta\left(\boldsymbol{x} i_{n+1} i_{n} i_{n-1} \cdots i_{2}\right)} \times \\
& \chi_{\left\{\boldsymbol{w}: \boldsymbol{w}=\boldsymbol{x} i_{n+1} i_{n} i_{n-1} \cdots i_{2} i_{1}\right\}}(\boldsymbol{y}) \\
& =\sum_{i_{1}, i_{2}, \cdots, i_{n+1}=1}^{d} \frac{e^{\left[\phi\left(f_{\boldsymbol{y}}(z)\right)+\phi\left(f_{\boldsymbol{x} i_{n+1} i_{n} \cdots i_{2}}(z)\right)+\cdots+\phi\left(f_{\boldsymbol{x} i_{n+1}}(z)\right)-(n+1) c\right]}}{\Theta(\boldsymbol{x}) \Theta\left(\boldsymbol{x} i_{n+1}\right) \Theta\left(\boldsymbol{x} i_{n+1} i_{n}\right) \cdots \Theta\left(\boldsymbol{x} i_{n+1} i_{n} \cdots i_{2}\right)} \times \\
& \chi_{\left\{\boldsymbol{w}: \boldsymbol{w}=\boldsymbol{x} i_{n+1} i_{n} i_{n-1} \cdots i_{2} i_{1}\right\}}(\boldsymbol{y})
\end{aligned}
$$




$$
=\sum_{i_{1}, i_{2}, \cdots, i_{n+1}=1}^{d} \frac{e^{\left[\phi\left(f_{\boldsymbol{y}}(z)\right)+\phi\left(f_{\boldsymbol{x} i_{n+1} i_{n} \cdots i_{2}}(z)\right)+\cdots+\phi\left(f_{\boldsymbol{x} i_{n+1}}(z)\right)-(n+1) c\right]}}{\Theta(\boldsymbol{x}) \Theta\left(\boldsymbol{x} i_{n+1}\right) \Theta\left(\boldsymbol{x} i_{n+1} i_{n}\right) \cdots \Theta\left(\boldsymbol{x} i_{n+1} i_{n} \cdots i_{2}\right)} \times
$$

Hence the claim is established.

Thus for $\boldsymbol{x}, \boldsymbol{y} \in \mathcal{W}$, and $i_{n}, i_{n-1}, \cdots, i_{2}, i_{1} \in \mathcal{A}$ we have

$$
p(n ; \boldsymbol{x}, \boldsymbol{y})= \begin{cases}\frac{e^{\left[\phi\left(f_{\boldsymbol{y}}(z)\right)+\phi\left(f_{\boldsymbol{x} i_{n} \cdots i_{2}}(z)\right)+\cdots+\phi\left(f_{\boldsymbol{x} i_{n}}(z)\right)-n c\right]}}{\Theta(\boldsymbol{x}) \Theta\left(\boldsymbol{x} i_{n}\right) \Theta\left(\boldsymbol{x} i_{n} i_{n-1}\right) \cdots \Theta\left(\boldsymbol{x} i_{n} i_{n-1} \cdots i_{2}\right)} & \text { if } \boldsymbol{y}=\boldsymbol{x} i_{n} \cdots i_{2} i_{1} \\ 0 & \text { otherwise }\end{cases}
$$

Now we are in a position to express the Martin kernel $k(\cdot, \cdot)$. For $\boldsymbol{x}, \boldsymbol{y} \in \mathcal{W}$, the numerator $g(\boldsymbol{x}, \boldsymbol{y})$ has the following form:

$$
\begin{aligned}
g(\boldsymbol{x}, \boldsymbol{y}) & =p(d(\boldsymbol{x}, \boldsymbol{y}) ; \boldsymbol{x}, \boldsymbol{y}) \\
& =\frac{e^{\left[\phi\left(f_{\boldsymbol{y}}(z)\right)+\phi\left(f_{\boldsymbol{y}[1]}(z)\right)+\cdots+\phi\left(f_{\boldsymbol{y}[d(\boldsymbol{x}, \boldsymbol{y})-1]}(z)\right)-d(\boldsymbol{x}, \boldsymbol{y}) c\right]}}{\Theta(\boldsymbol{y}[1]) \Theta(\boldsymbol{y}[2]) \cdots \Theta(\boldsymbol{y}[d(\boldsymbol{x}, \boldsymbol{y})-1]) \Theta(\boldsymbol{y}[d(\boldsymbol{x}, \boldsymbol{y})])}
\end{aligned}
$$

And the denominator $g(\emptyset, \boldsymbol{y})$ of the kernel becomes:

$$
g(\emptyset, \boldsymbol{y})=\frac{e^{\left[\phi\left(f_{\boldsymbol{y}}(z)\right)+\phi\left(f_{\boldsymbol{y}[1]}(z)\right)+\cdots+\phi\left(f_{\boldsymbol{y}[d(\boldsymbol{y})-1]}(z)\right)-d(\boldsymbol{y}) c\right]}}{\Theta(\boldsymbol{y}[1]) \Theta(\boldsymbol{y}[2]) \cdots \Theta(\boldsymbol{y}[d(\boldsymbol{y})-1]) \Theta(\emptyset)}
$$

Consequently, the Martin kernel $k(\boldsymbol{x}, \boldsymbol{y})$ is given by

$k(\boldsymbol{x}, \boldsymbol{y})= \begin{cases}\frac{\Theta(\boldsymbol{y}[d(\boldsymbol{x}, \boldsymbol{y})+1]) \Theta(\boldsymbol{y}[d(\boldsymbol{x}, \boldsymbol{y})+2]) \cdots \Theta(\boldsymbol{y}[d(\boldsymbol{y})-1]) \Theta(\emptyset)}{\left.\left.e^{\left[\phi\left(f_{\boldsymbol{y}[d(\boldsymbol{x}, \boldsymbol{y})]}(z)\right)+\phi\left(f \boldsymbol{y}_{\boldsymbol{y}[d(\boldsymbol{x}, \boldsymbol{y})+1]}(z)\right)+\cdots+\phi(f)\right.} \boldsymbol{y}[d(\boldsymbol{y})-1](z)\right)-d(\boldsymbol{x}) c\right]} & \text { if } \boldsymbol{x} \ll \boldsymbol{y} \\ 0 & \text { otherwise }\end{cases}$

As the Martin kernel obtained above is going to play an important role, we record the result as a theorem.

Theorem 55 For $\boldsymbol{x}, \boldsymbol{y} \in \mathcal{W}$, the Martin kernel has the following form:

$$
k(\boldsymbol{x}, \boldsymbol{y})= \begin{cases}\frac{\Theta(\boldsymbol{y}[d(\boldsymbol{x}, \boldsymbol{y})+1]) \Theta(\boldsymbol{y}[d(\boldsymbol{x}, \boldsymbol{y})+2]) \cdots \Theta(\boldsymbol{y}[d(\boldsymbol{y})-1]) \Theta(\emptyset)}{\left.\left.e^{\left[\phi\left(f_{\boldsymbol{y}[d(\boldsymbol{x}, \boldsymbol{y})]}(z)\right)+\phi\left(f_{\boldsymbol{y}[d(\boldsymbol{x}, \boldsymbol{y})+1]}(z)\right)+\cdots+\phi(f) \boldsymbol{y}[d(\boldsymbol{y})-1]\right.}(z)\right)-d(\boldsymbol{x}) c\right]} & \text { if } \boldsymbol{x} \ll \boldsymbol{y} \\ 0 & \text { otherwise }\end{cases}
$$




\subsection{Symbolic Space and Julia Set}

In this section we prove that a certain class of Julia sets can be identified with the one-sided shift space via a Lipschitz map.

Definition 56 Let $\left(X_{1}, d_{1}\right),\left(X_{2}, d_{2}\right)$ be metric spaces. A map $f: X_{1} \rightarrow$ $X_{2}$ is said to be Hölder continuous with exponent $\alpha$, or $\alpha$-Hölder, if there exist real constants $C, \epsilon>0$ such that

$$
d_{1}(x, y)<\epsilon \Rightarrow d_{2}(f(x), f(y)) \leq C\left(d_{1}(x, y)\right)^{\alpha},
$$

is said to be Lipschitz continuous if it is 1-Hölder, and biLipschitz if it is Lipschitz and has a Lipschitz inverse.

We need the following general result for our next theorem.

Proposition 57 Let $X$ be a locally compact Hausdorff space, let $K$ be a compact subset of $X$, and let $U$ be an open subset of $X$ that includes $K$. Then there is an open subset $V$ of $X$ that has a compact closure and satisfies $K \subset V \subset \bar{V} \subset U$.

Proof: We refer to Cohn [14].

Theorem 58 Let $f$ be a hyperbolic rational map of degree $d$, where $d \geq 2$. Let its Julia set $J(f)$ be totally disconnected. Then the map

$$
\Phi: \Sigma^{+} \rightarrow J(f)
$$

defined by

$$
\Phi\left(w_{1} w_{2} w_{3} \cdots\right):=\cap_{n \geq 1} f_{w_{1}} \circ \cdots \circ f_{w_{n}}\left(U_{\epsilon}\right),
$$

where $U_{\epsilon}$ is a sufficiently small open set containing $J(f)$ on which $f$ is expanding, is bijective and Lipschitz continuous. Moreover, the inverse map $\Phi^{-1}$ is also continuous.

Proof: The existence of $U_{\epsilon}$ follows from Proposition 57 simply by taking $X=\mathbb{C}_{\infty}, K=J(f)$ and $U=\mathbb{C}_{\infty}-P(f)$ and by using the fact that $f$ is hyperbolic. 
First, $\Phi$ is well defined: Since $f$ is expanding on $U_{\epsilon}$, all its inverse branches $f_{w_{i}}$ are contraction on $U_{\epsilon}$. Consequently, we have

$$
f_{w_{1}} \circ \cdots \circ f_{w_{n}}\left(f_{w_{n+1}}\left(U_{\epsilon}\right)\right) \subset f_{w_{1}} \circ \cdots \circ f_{w_{n}}\left(U_{\epsilon}\right)
$$

By using the compactness of $J$ and the fact that $\sigma\left(z, \mathbb{C}_{\infty}-U_{\epsilon}\right)>0$ for all $z \in J$, we can obtain a nonempty closed neighborhood $V \subset U_{\epsilon}$ of $J$ such that $\left\{f_{w_{1}} \circ \cdots \circ f_{w_{n}}(V)\right\}_{n \geq 1}$ forms a decreasing sequence of sets. As $f_{w_{1}} \circ \cdots \circ f_{w_{n}}(V)$ is compact, $\cap_{n \geq 1} f_{w_{1}} \circ \cdots \circ f_{w_{n}}(V)$ is a non-empty compact set. For $A \subset \mathbb{C}_{\infty}$, the diameter of $A$, $\operatorname{diam}(A)$, is defined by $\operatorname{diam}(A)=\sup _{z, z^{\prime} \in A} \sigma\left(z, z^{\prime}\right)$. Set $K=\max _{1 \leq i \leq d} \operatorname{Lip}\left(f_{w_{i}}\right)$. Then it follows that $\operatorname{diam}\left(f_{i}(A)\right) \leq K \operatorname{diam}(A)$. Hence

$$
\operatorname{diam}\left(f_{w_{1}} \circ \cdots \circ f_{w_{n}}\left(U_{\epsilon}\right)\right) \leq K^{n} \operatorname{diam}\left(U_{\epsilon}\right) .
$$

So $\operatorname{diam}\left(\cap_{n \geq 1} f_{w_{1}} \circ \cdots \circ f_{w_{n}}\left(U_{\epsilon}\right)\right)=0$. Thus $\cap_{n \geq 1} f_{w_{1}} \circ \cdots \circ f_{w_{n}}\left(U_{\epsilon}\right)$ contains only one point and the map $\Phi$ is well-defined.

Second, $\Phi$ is one-one: Let $w, w^{\prime} \in \Sigma^{+}, w \neq w^{\prime}$. Let $w, w^{\prime}$ differ at their $l$-th coordinate, that is, $w_{l} \neq w_{l}^{\prime}$. This implies that

$$
f_{w_{l}}\left(U_{\epsilon}\right) \cap f_{w_{l}^{\prime}}\left(U_{\epsilon}\right)=\phi .
$$

Since each $f_{w_{i}}$ is one-one it follows that

$$
f_{w_{1}} \circ f_{w_{2}} \circ \cdots \circ f_{w_{l}}\left(U_{\epsilon}\right) \cap f_{w_{1}} \circ f_{w_{2}} \circ \cdots \circ f_{w_{l}^{\prime}}\left(U_{\epsilon}\right)=\phi .
$$

Hence $\Phi(w) \neq \Phi\left(w^{\prime}\right)$, as required.

Third, $\Phi$ is onto: Let $z_{0} \in J(f)$. We produce $w \in \Sigma^{+}$such that $\Phi(w)=z_{0}$. Since $J(f) \subset \cup_{i=1}^{d} f_{i}\left(U_{\epsilon}\right)$, where $f_{i}(i=1,2, \cdots, d)$ are inverse branches of $f$, it follows that there exist unique $i \in\{1,2, \cdots, d\}$ such that $z_{0} \in f_{i}\left(U_{\epsilon}\right)$. Now, the proof continues by induction.

Assume $z_{0} \in S:=f_{w_{l}} \circ f_{w_{2}} \circ \cdots \circ f_{w_{n}}\left(U_{\epsilon}\right) \cap J(f)$. Since

$$
\begin{aligned}
& {\left[\cup_{j=1}^{d} f_{w_{1}} \circ f_{w_{2}} \circ \cdots \circ f_{w_{n}} \circ f_{j}\left(U_{\epsilon}\right)\right] \cap J(f) } \\
= & f_{w_{1}} \circ f_{w_{2}} \circ \cdots \circ f_{w_{n}}\left[\cup_{j=1}^{d} f_{j}\left(U_{\epsilon}\right)\right] \cap J(f) \\
= & f_{w_{1}} \circ f_{w_{2}} \circ \cdots \circ f_{w_{n}}\left(U_{\epsilon}\right) \cap J(f),
\end{aligned}
$$


it follows that $z_{0}$ is in $f_{w_{1}} \circ f_{w_{2}} \circ \cdots \circ f_{w_{n}} \circ f_{w_{n+1}}\left(U_{\epsilon}\right) \cap J(f)$ for a unique $w_{n+1}$ in $\{1,2, \cdots, d\}$. Hence $\Phi\left(w_{1} w_{2} \cdots\right)=z_{0}$.

Fourth, $\Phi$ is continuous, and in fact Lipschitz: Let $w=w_{1} w_{2} w_{3} \cdots$ be an arbitrary but fixed point in $\Sigma^{+}$. We show that $\Phi$ is continuous at $w$.

Consider the following set of points in $\Sigma^{+}$:

$$
\mathcal{N}(w):=\left\{v \in \Sigma^{+}: v_{i}=w_{i}, i=1,2, \cdots, m\right\}
$$

That means, the set $\mathcal{N}(w)$ consists of points in $\Sigma^{+}$that coincide with $w$ up to its $m$-th coordinate (not that we can make $m$ as large as we want). Clearly for $v \in \mathcal{N}(w)$ we have

$$
\Phi(w), \Phi(v) \in f_{w_{1}} \circ f_{w_{2}} \circ \cdots \circ f_{w_{m}}\left(U_{\epsilon}\right)
$$

Hence

$$
\begin{aligned}
\sigma(\Phi(w), \Phi(v)) & \leq \operatorname{diam}\left(f_{w_{1}} \circ f_{w_{2}} \circ \cdots \circ f_{w_{m}}\left(U_{\epsilon}\right)\right) \\
& \leq K^{m} \operatorname{diam}\left(U_{\epsilon}\right)
\end{aligned}
$$

This immediately implies that $\Phi$ is continuous (in fact uniformly continuous) on $\Sigma^{+}$. And that $\Phi$ is Lipschitz also follows from the same inequality simply by choosing $r, C>0$ such that $K<r<1$ and $C \geq \operatorname{diam}\left(U_{\epsilon}\right) / r$; since then we have

$$
\sigma(\Phi(w), \Phi(v)) \leq r^{m} r C \leq C \rho(w, v)
$$

Finally, $\Phi^{-1}$ is continuous: This follows from a general result (see Aggarwal [1]) that states that if $\Phi$ is a continuous bijection from a compact topological space $X$ onto a Hausdorff space $Y$, then $\Phi$ is a homeomorphism.

Corollary 59 Let $\sigma: \Sigma^{+} \rightarrow \Sigma^{+}$be the left shift map on $\Sigma^{+}$. Then $\sigma$ is topologically conjugate to the rational map $f: J \rightarrow J$. In other words, 
we have $\Phi \circ \sigma=f \circ \Phi$; that is, the following diagram commutes:

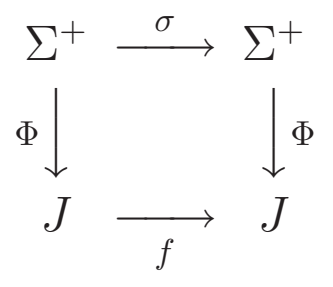

Corollary 60 The topological entropy $h_{\text {top }}(f)$ of the map $f: J \rightarrow J$ is $\log d$.

Corollary 61 For a continuous map $\psi: J \rightarrow \mathbb{R}$ we have

$$
P(f, \psi)=P(\sigma, \psi \circ \Phi) .
$$

Proof: This follows from Theorem 9.8 at Walter [55].

Corollary 62 The measure, say $\mu_{m}$, of maximal entropy for the rational map $f: J \rightarrow J$ is equal to the image measure of the $(1 / d, \cdots, 1 / d)$ Bernoulli measure, say $\mu_{b}$, under the mapping $\Phi$; that is: $\mu_{b} \circ \Phi^{-1}=\mu_{m}$.

Proof: The existence of a unique measure of maximal entropy for the rational map $f: J \rightarrow J$ is established by Mañé [43]. Now the corollary follows from Theorem 40 and Theorem 41.

Corollary 63 Let $J$ be the Julia set in the above Theorem 58. Then $\left(J,\{1,2, \cdots, d\},\left\{f_{i}\right\}_{i=1}^{d}\right)$, where the $f_{i}$ are the inverse branches of the rational map $f$, is a postcritically finite self-similar structure.

Proof: The proof follows from Proposition 11.

\subsection{Julia set as a Martin Boundary}

In this section we identify the Julia set with the Martin boundary $M$ of the transition probability function defined in Section 3.1. Since by Theorem 58 the Julia set is homeomorphic to the space $\Sigma^{+}$, it suffices to show the existence of a homeomorphism

$$
H: \Sigma^{+} \rightarrow M
$$


Lemma 64 Let $x=\left\{x_{k}\right\}$ be an infinite sequence of letters and define

$$
\boldsymbol{w}_{n}=x_{1} x_{2} x_{3} \cdots x_{n}, \quad n \geq 1 .
$$

Then $T_{0}(x)=\left\{\boldsymbol{w}_{n}\right\}$ is a Cauchy sequence in $\mathcal{W}=(\mathcal{W}, \rho)$.

Proof: Let $\boldsymbol{u}=u_{1} u_{2} \cdots u_{m}$ be any word. We may assume that $d\left(\boldsymbol{w}_{n}\right)=n\left(\right.$ also $\left.d\left(\boldsymbol{w}_{n^{\prime}}\right)=n^{\prime}\right)$ is sufficiently large, say $d\left(\boldsymbol{w}_{n}\right) \geq m+1$. If $\boldsymbol{w}_{m} \neq \boldsymbol{u}$, then $\boldsymbol{u} \nless \boldsymbol{w}_{n}$ and consequently $k\left(\boldsymbol{u}, \boldsymbol{w}_{n}\right)=0$ for any $n \geq m+1$. Thus $\rho\left(\boldsymbol{w}_{n}, \boldsymbol{w}_{n^{\prime}}\right) \rightarrow 0$ as $n, n^{\prime} \rightarrow \infty$.

Without loss of generality we may assume that $n^{\prime}>n$. If $\boldsymbol{w}_{m}=\boldsymbol{u}$, then $\boldsymbol{w}_{n}$ (also $\left.\boldsymbol{w}_{n^{\prime}}\right)$ is a successor of $\boldsymbol{u}$. Since $k\left(\boldsymbol{u}, \boldsymbol{w}_{n}\right)$ and $k\left(\boldsymbol{u}, \boldsymbol{w}_{n^{\prime}}\right)$ has got the same value according to Theorem 55 for all $n, n^{\prime} \geq m+1$, it also follows that $\rho\left(\boldsymbol{w}_{n}, \boldsymbol{w}_{n^{\prime}}\right) \rightarrow 0$ as $n, n^{\prime} \rightarrow \infty$.

In view of Lemma 64 we can define the map

$$
H: \Sigma^{+} \rightarrow M
$$

by setting $H(x):=\left\{\boldsymbol{w}_{n}: n \geq 1\right\}$, where $\boldsymbol{w}_{n}=x_{1} x_{2} \cdots x_{n}$ and $x=$ $\left(x_{m}\right) \in \Sigma^{+}$, and where $\left\{\boldsymbol{w}_{n}: n \geq 1\right\}$ denotes the equivalence class of the Cauchy sequence $\left\{\boldsymbol{w}_{n}: n \geq 1\right\}$. We shall prove that $H$ is a homeomorphism.

Lemma 65 The map $H: \Sigma^{+} \rightarrow M$ defined above is one-to-one.

Proof: Suppose $x, y \in \Sigma^{+}$, and $x \neq y$. We need to show that $H(x) \neq H(y)$. Define

$$
\boldsymbol{x}_{n}:=x_{1} x_{2} \cdots x_{n} \text { and } \boldsymbol{y}_{n}:=y_{1} y_{2} \cdots y_{n}
$$

It follows from Lemma 64 that both the sequences $\left(\boldsymbol{x}_{n}\right)$ and $\left(\boldsymbol{y}_{n}\right)$ are Cauchy in $(\mathcal{W}, \rho)$. It suffices to prove that

$$
\lim _{n \rightarrow \infty} \rho\left(\boldsymbol{x}_{n}, \boldsymbol{y}_{n}\right) \neq 0
$$


As $x \neq y$, define $k:=\min \left\{l \geq 1: x_{l} \neq y_{l}\right\}$. Now let us put $\boldsymbol{u}^{\prime}=$ $x_{1} x_{2} \cdots x_{k-1} x_{k}\left(=y_{1} y_{2} \cdots y_{k-1} x_{k}\right)$. Then

$$
\begin{aligned}
& \sum_{\boldsymbol{u} \in \mathcal{W}} a(\boldsymbol{u}) \frac{\left|k\left(\boldsymbol{u}, \boldsymbol{y}_{n}\right)-k\left(\boldsymbol{u}, \boldsymbol{x}_{n}\right)\right|}{1+\left|k\left(\boldsymbol{u}, \boldsymbol{y}_{n}\right)-k\left(\boldsymbol{u}, \boldsymbol{x}_{n}\right)\right|} \\
&= a\left(\boldsymbol{u}^{\prime}\right) \frac{\left|k\left(\boldsymbol{u}^{\prime}, \boldsymbol{y}_{n}\right)-k\left(\boldsymbol{u}^{\prime}, \boldsymbol{x}_{n}\right)\right|}{1+\left|k\left(\boldsymbol{u}^{\prime}, \boldsymbol{y}_{n}\right)-k\left(\boldsymbol{u}^{\prime}, \boldsymbol{x}_{n}\right)\right|}+ \\
& \sum_{\boldsymbol{u} \in \mathcal{W}, \boldsymbol{u} \neq \boldsymbol{u}^{\prime}} a(\boldsymbol{u}) \frac{\left|k\left(\boldsymbol{u}, \boldsymbol{y}_{n}\right)-k\left(\boldsymbol{u}, \boldsymbol{x}_{n}\right)\right|}{1+\left|k\left(\boldsymbol{u}, \boldsymbol{y}_{n}\right)-k\left(\boldsymbol{u}, \boldsymbol{x}_{n}\right)\right|} \\
& \geq a\left(\boldsymbol{u}^{\prime}\right) \frac{\left|k\left(\boldsymbol{u}^{\prime}, \boldsymbol{y}_{n}\right)-k\left(\boldsymbol{u}^{\prime}, \boldsymbol{x}_{n}\right)\right|}{1+\left|k\left(\boldsymbol{u}^{\prime}, \boldsymbol{y}_{n}\right)-k\left(\boldsymbol{u}^{\prime}, \boldsymbol{x}_{n}\right)\right|}
\end{aligned}
$$

Now as $\boldsymbol{u}^{\prime} \nless \boldsymbol{y}_{n}$, we have $k\left(\boldsymbol{u}^{\prime}, \boldsymbol{y}_{n}\right)=0$, whereas $k\left(\boldsymbol{u}^{\prime}, \boldsymbol{x}_{n}\right) \neq 0$ since $\boldsymbol{u}^{\prime} \ll \boldsymbol{x}_{n}$. Thus the above inequality takes the following form:

$$
\sum_{\boldsymbol{u} \in \mathcal{W}} a(\boldsymbol{u}) \frac{\left|k\left(\boldsymbol{u}, \boldsymbol{y}_{n}\right)-k\left(\boldsymbol{u}, \boldsymbol{x}_{n}\right)\right|}{1+\left|k\left(\boldsymbol{u}, \boldsymbol{y}_{n}\right)-k\left(\boldsymbol{u}, \boldsymbol{x}_{n}\right)\right|} \geq a\left(\boldsymbol{u}^{\prime}\right) \frac{\left|k\left(\boldsymbol{u}^{\prime}, \boldsymbol{x}_{n}\right)\right|}{1+\left|k\left(\boldsymbol{u}^{\prime}, \boldsymbol{x}_{n}\right)\right|}
$$

Since $a\left(\boldsymbol{u}^{\prime}\right)>0$ and the value of $k\left(\boldsymbol{u}^{\prime}, \boldsymbol{x}_{n}\right)$ is also positive and constant for all $n$, it follows that

$$
\lim _{n} \rho\left(\boldsymbol{x}_{n}, \boldsymbol{y}_{n}\right) \neq 0
$$

Lemma 66 The map $H: \Sigma^{+} \rightarrow M$ defined above is surjective.

Proof: Let $\left\{\boldsymbol{w}_{n}\right\}$ be a Cauchy sequence in $\mathcal{W}$. Then, since $\mathcal{A}$ is a finite set, there exists a subsequence $\left\{\boldsymbol{w}_{n(1, k)} ; k \geq 1\right\}$ such that the first letter of all $\boldsymbol{w}_{n(1, k)}, k \geq 1$, is $x_{1} \in \mathcal{A}$. Next we can extract a subsequence $\left\{\boldsymbol{w}_{n(2, k)} ; k \geq 1\right\}$ of $\left\{\boldsymbol{w}_{n(1, k)} ; k \geq 1\right\}$ such that the second letter of all $\boldsymbol{w}_{n(2, k)}, k \geq 1$, is $x_{2} \in \mathcal{A}$. Similarly we can extract a subsequence $\left\{\boldsymbol{w}_{n(j+1, k)} ; k \geq 1\right\}$ of $\left\{\boldsymbol{w}_{n(j, k)} ; k \geq 1\right\}$ such that the $(j+1)$-th letter of all $\boldsymbol{w}_{n(j+1, k)}, k \geq 1$, is $x_{j+1} \in \mathcal{A}$. Define $\boldsymbol{w}_{k}^{0}=\boldsymbol{w}_{n(k, k)}, k \geq 1$. Then $\boldsymbol{w}_{k}^{0}$, being a subsequence of $\left\{\boldsymbol{w}_{n}\right\}$, is a Cauchy sequence. Moreover, $\left\{\boldsymbol{w}_{k}^{0}\right\}$ is equivalent to $\left\{\boldsymbol{w}_{n}\right\}$ : 


$$
\begin{aligned}
\rho\left(\boldsymbol{w}_{k}, \boldsymbol{w}_{k}^{0}\right) & =\left|2^{-d\left(\boldsymbol{w}_{k}\right)}-2^{-d\left(\boldsymbol{w}_{k}^{0}\right)}\right|+\sum_{\boldsymbol{u} \in \mathcal{W}} a(\boldsymbol{u}) \frac{\left|k\left(\boldsymbol{u}, \boldsymbol{w}_{k}^{0}\right)-k\left(\boldsymbol{u}, \boldsymbol{w}_{k}\right)\right|}{1+\left|k\left(\boldsymbol{u}, \boldsymbol{w}_{k}^{0}\right)-k\left(\boldsymbol{u}, \boldsymbol{w}_{k}\right)\right|} \\
& \leq\left|2^{-d\left(\boldsymbol{w}_{k}\right)}-2^{-d\left(\boldsymbol{w}_{k}^{0}\right)}\right|+\sum_{\boldsymbol{u} \in \mathcal{W}, d(\boldsymbol{u}) \geq p} a(\boldsymbol{u}) \\
& +\sum_{\boldsymbol{u} \in \mathcal{W}, d(\boldsymbol{u})<p}\left|k\left(\boldsymbol{u}, \boldsymbol{w}_{k}^{0}\right)-k\left(\boldsymbol{u}, \boldsymbol{w}_{k}\right)\right|
\end{aligned}
$$

Clearly the first term on the right tends to zero as $k \rightarrow \infty$. The second term can be made arbitrarily small since $\sum_{\boldsymbol{u} \in \mathcal{W}} a(\boldsymbol{u})=1$. And since $\left\{\boldsymbol{w}_{k}\right\}$ is Cauchy, $\rho\left(\boldsymbol{w}_{k}, \boldsymbol{w}_{k}^{0}\right) \rightarrow 0$ as $k \rightarrow \infty$. Thus, by uniform continuity of $k(\boldsymbol{u}, \cdot)$ each term of the finite sum tends to zero. Hence $\left\{\boldsymbol{w}_{k}\right\}$ and $\left\{\boldsymbol{w}_{k}^{0}\right\}$ belong to the same equivalence class of Cauchy sequences.

Now consider the sequence $\left\{\boldsymbol{v}_{n}\right\}$, where $\boldsymbol{v}_{n}=x_{1} x_{2} \cdots x_{n}$. By Lemma 64, $\left\{\boldsymbol{v}_{n}\right\}$ is a Cauchy sequence. We claim that $\left\{\boldsymbol{v}_{n}\right\}$ and $\left\{\boldsymbol{w}_{k}^{0}\right\}$ are equivalent, that is, $\lim _{k \rightarrow \infty} \rho\left(\boldsymbol{w}_{k}^{0}, \boldsymbol{v}_{k}\right)=0$. Clearly, $\left|2^{-d\left(\boldsymbol{w}_{k}^{0}\right)}-2^{-d\left(\boldsymbol{v}_{k}\right)}\right| \rightarrow 0$ since $d\left(\boldsymbol{w}_{k}^{0}\right)$ and $d\left(\boldsymbol{v}_{k}\right)$ tend to infinity as $k \rightarrow \infty$. Now

$$
\begin{aligned}
& \sum_{\boldsymbol{u} \in \mathcal{W}} a(\boldsymbol{u}) \frac{\left|k\left(\boldsymbol{u}, \boldsymbol{v}_{k}\right)-k\left(\boldsymbol{u}, \boldsymbol{w}_{k}^{0}\right)\right|}{1+\left|k\left(\boldsymbol{u}, \boldsymbol{v}_{k}\right)-k\left(\boldsymbol{u}, \boldsymbol{w}_{k}^{0}\right)\right|} \leq \sum_{\boldsymbol{u} \in \mathcal{W}, d(\boldsymbol{u}) \geq p} a(\boldsymbol{u})+ \\
& \sum_{\boldsymbol{u} \in \mathcal{W}, d(\boldsymbol{u})<p}\left|k\left(\boldsymbol{u}, \boldsymbol{v}_{k}\right)-k\left(\boldsymbol{u}, \boldsymbol{w}_{k}^{0}\right)\right|
\end{aligned}
$$

Depending on the situation of $\boldsymbol{v}_{k}$, whether or not $\boldsymbol{v}_{k}^{\prime}$ s are successors of the $\boldsymbol{u}^{\prime}$ s ( if so, $\boldsymbol{w}_{k}^{0}$ 's are also successors of $\boldsymbol{u}^{\prime}$ s ), the second term on the right is always zero. Thus it follows that $\left\{\boldsymbol{v}_{n}\right\}$ is equivalent to $\left\{\boldsymbol{w}_{k}\right\}$. So they are in the same equivalence class of Cauchy sequences. Now, by construction, it follows that if we take $x=x_{1} x_{2} x_{3} \cdots \in \Sigma^{+}$, then $H(x)=\left\{\boldsymbol{w}_{n}: n \geq 1\right\}$. Hence $H$ is surjective.

Lemma 67 The map $H: \Sigma^{+} \rightarrow M$ defined above is continuous. 
Proof: Let $\left\{x^{(n)}\right\}$ be a convergent sequence in $\Sigma^{+}: x^{(n)} \rightarrow u$. We show $H\left(x^{(n)}\right) \rightarrow H(u)$ as $n \rightarrow \infty$, where $H\left(x^{(n)}\right)=\left\{\overline{\boldsymbol{x}}_{k}^{(n)}: k \geq 1 \tilde{\}}\right.$, $H(u)=\left\{\overline{\boldsymbol{u}}_{k}: k \geq 1\right\}$ and $\overline{\boldsymbol{x}}_{k}^{(n)}:=x_{1}^{(n)} x_{2}^{(n)} \cdots x_{k}^{(n)}, \overline{\boldsymbol{u}}_{k}:=u_{1} u_{2} \cdots u_{k}$. Then

$$
\begin{aligned}
& \rho\left(H\left(x^{(n)}\right), H(u)\right)=\lim _{k \rightarrow \infty} \rho\left(\overline{\boldsymbol{x}}_{k}^{(n)}, \overline{\boldsymbol{u}}_{k}\right) \\
&= \lim _{k \rightarrow \infty}\left(\left|2^{-d\left(\overline{\boldsymbol{x}}_{k}^{(n)}\right)}-2^{-d\left(\overline{\boldsymbol{u}}_{k}\right)}\right|+\sum_{\boldsymbol{v} \in \mathcal{W}} a(\boldsymbol{v}) \frac{\left|k\left(\boldsymbol{v}, \overline{\boldsymbol{u}}_{k}\right)-k\left(\boldsymbol{v}, \overline{\boldsymbol{x}}_{k}^{(n)}\right)\right|}{1+\left|k\left(\boldsymbol{v}, \overline{\boldsymbol{u}}_{k}\right)-k\left(\boldsymbol{v}, \overline{\boldsymbol{x}}_{k}^{(n)}\right)\right|}\right) \\
& \leq \lim _{k \rightarrow \infty}\left|2^{-d\left(\overline{\boldsymbol{x}}_{k}^{(n)}\right)}-2^{-d\left(\overline{\boldsymbol{u}}_{k}\right)}\right|+\sum_{\boldsymbol{v} \in \mathcal{W} d(\boldsymbol{v}) \geq p} a(\boldsymbol{v})+ \\
& \lim _{k \rightarrow \infty} \sum_{\boldsymbol{v} \in \mathcal{W}, d(\boldsymbol{v})<p}\left|k\left(\boldsymbol{v}, \overline{\boldsymbol{u}}_{k}\right)-k\left(\boldsymbol{v}, \overline{\boldsymbol{x}}_{k}^{(n)}\right)\right|
\end{aligned}
$$

Hence the result follows by taking the limit on both sides as $n \rightarrow \infty$.

We are now in a position to prove a representation theorem for harmonic functions. We recall from Dynkin [22] that every excessive function $h: \mathcal{W} \rightarrow \mathbb{R}_{+}$has a representation

$$
h(\boldsymbol{v})=\int_{J \cup \mathcal{W}} k(\boldsymbol{v}, y) \mu_{h}(d y)
$$

for some finite measure $\mu_{h}$. Moreover, each function $k_{y}$ defined by $k_{y}(\boldsymbol{v})=k(\boldsymbol{v}, y)$ is excessive. The space of exits consists of those $\xi \in J$ for which $\mu_{k_{\xi}}$ is the unit mass in $\xi$.

Theorem 68 (1) The function $\boldsymbol{w} \mapsto k_{y}(\boldsymbol{w})=k(\boldsymbol{w}, y)$ is P-harmonic on $\mathcal{W}$ for every $y \in J$.

(2) $J$ is the space of exits.

(3) For every $P$-excessive function $h \geq 0$ there exists a unique finite measure $\mu_{h}$ on $\mathcal{W} \cup J$ such that

$$
h(\boldsymbol{w})=\int_{J \cup \mathcal{W}} k(\boldsymbol{w}, y) \mu_{h}(d y)
$$


(4) For every $P$-harmonic function $h \geq 0$ there exists a unique finite measure $\mu_{h}$ on $J$ such that

$$
h(\boldsymbol{w})=\int_{J} k(\boldsymbol{w}, y) \mu_{h}(d y)
$$

(5) For every bounded P-harmonic function $h \geq 0, \mu_{h}$ is absolutely continuous with respect to $\mu_{1}$ with bounded Radon-Nikodym derivative $\phi$ such that

$$
h(\boldsymbol{w})=\int_{J} k(\boldsymbol{w}, y) \phi(y) \mu_{1}(d y) .
$$

We also have

$$
\begin{aligned}
& \lim _{n \rightarrow \infty} h\left(X_{n}\right)=\phi\left(X_{\infty}\right) \quad P^{\boldsymbol{w}} \text {-almost sure } \forall \boldsymbol{w} \in \mathcal{W} \\
& \exists X_{\infty} \text { such that } h(\boldsymbol{w})=E^{\boldsymbol{w}}\left[\phi\left(X_{\infty}\right)\right] \quad \forall \boldsymbol{w} \in \mathcal{W} .
\end{aligned}
$$

(6) Let $\phi$ be a non-negative $\mu_{1}$-integrable function on $J$. Then

$$
h(\boldsymbol{w})=\int_{J} k(\boldsymbol{w}, y) \phi(y) \mu_{1}(d y)
$$

defines a P-harmonic function on $\mathcal{W}$ and

$$
\lim _{n \rightarrow \infty} h\left(X_{n}\right)=\phi\left(X_{\infty}\right) P^{w} \text {-almost sure } \forall \boldsymbol{w} \in \mathcal{W} .
$$

Here $\left\{X_{n} \mid n \in \mathbb{N}\right\}$ denotes the associated Markov chain and $P^{w}$ is the probability measure concentrated on the paths starting from $\boldsymbol{w}$, given by

$$
P^{\boldsymbol{w}}\left[X_{0}=x_{0}, X_{1}=x_{1}, \cdots, X_{n}=x_{n}\right]=\delta_{\boldsymbol{w}, \boldsymbol{x}_{0}} p\left(\boldsymbol{x}_{0}, \boldsymbol{x}_{1}\right) p\left(\boldsymbol{x}_{1}, \boldsymbol{x}_{2}\right) \cdots p\left(\boldsymbol{x}_{n-1}, \boldsymbol{x}_{n}\right)
$$


Proof: (1) Let $y \in J$ and $\boldsymbol{v} \in \mathcal{W}$ be fixed. Then

$$
\begin{aligned}
P k_{y}(\boldsymbol{v}) & =\sum_{\boldsymbol{u} \in \mathcal{W}} p(\boldsymbol{v}, \boldsymbol{u}) k_{y}(\boldsymbol{u}) \\
& =\sum_{\boldsymbol{u} \in \mathcal{W}} p(\boldsymbol{v}, \boldsymbol{u}) \lim _{\boldsymbol{w} \rightarrow y} \frac{g(\boldsymbol{u}, \boldsymbol{w})}{g(\emptyset, \boldsymbol{w})} \\
& =\sum_{\boldsymbol{u} \in \mathcal{W}} \lim _{\boldsymbol{w} \rightarrow y} \frac{\sum_{n=0}^{\infty} p(\boldsymbol{v}, \boldsymbol{u}) p(n, \boldsymbol{u}, \boldsymbol{w})}{g(\emptyset, \boldsymbol{w})} \\
& =\lim _{\boldsymbol{w} \rightarrow y} \frac{\sum_{n=0}^{\infty} p(n+1, \boldsymbol{v}, \boldsymbol{w})}{g(\emptyset, \boldsymbol{w})} \\
& =\lim _{\boldsymbol{w} \rightarrow y} \frac{g(\boldsymbol{v}, \boldsymbol{w})-\delta_{\boldsymbol{v}}(\boldsymbol{w})}{g(\emptyset, \boldsymbol{w})} \\
& =k_{y}(\boldsymbol{v})
\end{aligned}
$$

Hence $k_{y}$ is $P$-harmonic.

(2) Let $y \in J$. Since $k_{y}$ is $P$-harmonic, by Dynkin [22] the measure $\mu_{k_{y}}$ has its support in $J$. Therefore it suffices to show that

$$
J \backslash\{y\}=\bigcup_{\boldsymbol{v} \in \mathcal{W} ; k(\boldsymbol{v}, y)=0}\{\xi \in J: k(\boldsymbol{v}, \xi)>0\} .
$$

Indeed, if $\boldsymbol{v} \in \mathcal{W}$ and $k(\boldsymbol{v}, y)=0$, then

$$
0=k(\boldsymbol{v}, y)=\int_{J} k(\boldsymbol{v}, \xi) \mu_{k_{y}}(d \xi),
$$

so that $\mu_{k_{y}}(\xi: k(\boldsymbol{v}, \xi)>0)=0$.

Let $\xi \in J, \xi \neq y$. Express $\Phi^{-1}(\xi)=\xi_{1} \xi_{2} \cdots$ and $\Phi^{-1}(y)=y_{1} y_{2} \cdots$ as the two points of $\Sigma^{+}$by using the homeomorphism $\Phi$. Let $s$ denote the smallest integer for which $\xi_{s} \neq y_{s}$. Consider $\boldsymbol{v}:=\xi_{1} \xi_{2} \cdots \xi_{k}$ and $\boldsymbol{u}=y_{1} y_{2} \cdots y_{k}$ for some $k>s$. Then obviously $k(\boldsymbol{v}, \boldsymbol{u})=0$ and hence also $k(\boldsymbol{v}, y)=0$. Since $k(\boldsymbol{v}, \xi)>0$ by construction of $\boldsymbol{v}$, it follows that equation (3.7) holds.

The remaining parts follows from Dynkin [22]. 


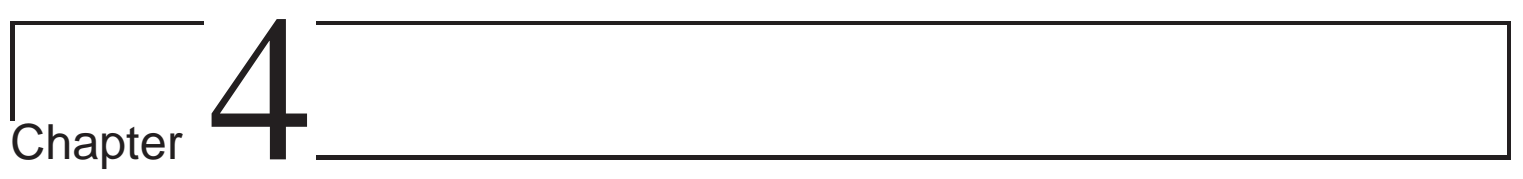

\section{Various Measures on the Julia Set}

In this chapter we study different measures on our totally disconnected Julia set identified as a Martin boundary of a certain Markov chain on the word space $\mathcal{W}$. In Section $\S 4.1$ we prove that the harmonic measure $\mu_{1}$ (in the sense of Dynkin [22]) on the Julia set $J$ is the image measure of a nonatomic, quasi-invariant, conservative measure $\nu$ on the one-sided shift space $\Sigma^{+}$. We will also show that this quasiinvariant measure $\nu$ is a Gibbs measure for a certain potential $\psi$ on $\Sigma^{+}$. In Section $\S 4.2$, we then prove that the measure $\nu$ gives rise to an equilibrium measure $\gamma=h \nu$ (where $h$ is the eigenfunction of a suitable transfer operator). Next, in Section $\S 4.3$ we show that our Julia set is non-polar and then, by using that property, we prove that the logarithmic potential $p_{\mu}(z)$, for $z \in J$, is a constant function, where $\mu$ is the equilibrium measure supported on $J$. As a consequence of these results it will turn out that the Fatou domain $D=\mathbb{C}_{\infty} \backslash J$ is regular and we have a unique solution for the Dirichlet problem correspoding to this domain $D$ in our case.

\subsection{Quasi-invariant Measure on the Julia Set}

We have seen that the totally disconnected Julia set $J$ of a hyperbolic rational map can be identified with the Martin boundary $M$ of a certain 
Markov chain and in the sequel we will not distinguish between them.

Define $h_{n}: \Sigma^{+} \mapsto \mathcal{W}, n \in \mathbb{N}$ by

$$
h_{n}(x):=x_{1} x_{2} \cdots x_{n} \in \mathcal{W}_{n}
$$

for $x=x_{1} x_{2} \cdots \in \Sigma^{+}$.

Theorem 69 The harmonic measure $\mu_{1}$ on $J$ in Theorem 68 coincides with the measure $\mu:=\nu \circ H^{-1}$ (called image measure of $\nu$ under the mapping $H$ ), where $\nu$ is the measure on $\Sigma^{+}$such that for $\boldsymbol{v} \in \mathcal{W}$

$$
\nu([\boldsymbol{v}])=\frac{\prod_{i=0}^{d(\boldsymbol{v})-1} e^{\left[\phi\left(f_{\boldsymbol{v}[i]}(z)\right)\right]}}{\sum_{l=1}^{d} e^{\phi\left(f_{\boldsymbol{v}[1] l}(z)\right)} \sum_{l=1}^{d} e^{\phi\left(f_{\boldsymbol{v}[2] l}(z)\right)} \cdots \sum_{l=1}^{d} e^{\phi\left(f_{l}(z)\right)}}
$$

Proof: By Theorem 68, the harmonic measure $\mu_{1}$ is uniquely determined by

$$
1=\int_{J} k(\boldsymbol{v}, \xi) \mu_{1}(d \xi), \quad \forall \boldsymbol{v} \in \mathcal{W}
$$

From the transformation rule we know that

$$
\int_{J} k(\boldsymbol{v}, y) \mu(d y)=\int_{\Sigma^{+}} k(\boldsymbol{v}, H(\omega)) \nu(d \omega)
$$

for any fixed $\boldsymbol{v} \in \mathcal{W}$.

Therefore it suffices to show that for every fixed $\boldsymbol{v} \in \mathcal{W}$ we have

$$
\int_{\Sigma^{+}} k(\boldsymbol{v}, H(\omega)) \nu(d \omega)=1
$$

We note that for every $\boldsymbol{v} \in \mathcal{W}$ we have

$$
k(\boldsymbol{v}, H(\omega))=\lim _{n \rightarrow \infty} k\left(\boldsymbol{v}, h_{n}(\omega)\right)
$$

and

$$
k\left(\boldsymbol{v}, h_{n}(\omega)\right)=\frac{\Theta(\boldsymbol{v}[1]) \Theta(\boldsymbol{v}[2]) \cdots \Theta(\emptyset)}{\prod_{i=0}^{d(\boldsymbol{v})-1} e^{\left[\phi\left(f_{\boldsymbol{v}[i]}(z)\right)-c\right]}}
$$

for all $n \geq 1$. 
Thus for every $\boldsymbol{v} \in \mathcal{W}$ we have

$$
\begin{aligned}
& \int_{\Sigma^{+}} k(\boldsymbol{v}, H(\omega)) \nu(d \omega) \\
= & \int_{\Sigma^{+}} \lim _{n \rightarrow \infty} k\left(\boldsymbol{v}, h_{n}(\omega)\right) \nu(d \omega) \\
= & \int_{\Sigma^{+}} \frac{\Theta(\boldsymbol{v}[1]) \Theta(\boldsymbol{v}[2]) \cdots \Theta(\emptyset)}{\prod_{i=0}^{d(\boldsymbol{v})-1} e^{\left[\phi\left(f_{\boldsymbol{v}[i]}(z)\right)-c\right]}} \nu(d \omega) \\
= & \frac{\Theta(\boldsymbol{v}[1]) \Theta(\boldsymbol{v}[2]) \cdots \Theta(\emptyset)}{\prod_{i=0}^{d(\boldsymbol{v})-1} e^{\left[\phi\left(f_{\boldsymbol{v}[i]}(z)\right)-c\right]}} \int_{[\boldsymbol{v}]} \nu(d \omega)
\end{aligned}
$$

The last equality holds since $k\left(\boldsymbol{v}, h_{n}(\omega)\right)=0$ if $\omega$ does not belong to the cylinder set $[\boldsymbol{v}]$. Once the expression outside the integral sign is simplified, it follows that

$$
\int_{\Sigma^{+}} k(\boldsymbol{v}, H(\omega)) \nu(d \omega)=1
$$

Lemma $70 \nu$ is a $\sigma$-finite, nonatomic measure on $\Sigma^{+}$.

Proof: $\nu$, being a probability measure, is obviously $\sigma$-finite. By expressing a point $x \in \Sigma^{+}$as the intersection of a sequence of decreasing cylinder sets, it is easy to see that $\nu(\{x\})=0$.

Definition 71 A probability measure $\mu$ on $\Sigma^{+}$is quasi-invariant if $\sigma^{*} \mu \ll \mu$ and $\mu \ll \sigma^{*} \mu$, where $\sigma^{*} \mu$ is the image measure of $\mu$ under the map $\sigma$.

We will use the following two results to prove the quasi-invariance of our measure $\nu$ :

Lemma 72 Let $(X, \mathcal{A})$ be a measurable space, let $m_{1}$ be a measure on $(X, \mathcal{A})$, and let $m_{2}$ be a finite measure on $(X, \mathcal{A})$. Then $m_{2} \ll m_{1}$ if and only if for each $\epsilon>0$ there exists a positive $\delta$ such that each $\mathcal{A}$-measurable set $A$ that satisfies $m_{1}(A)<\delta$ also satisfies $m_{2}(A)<\epsilon$. 
Proof: We refer to Cohn [14].

Lemma 73 If $0<t_{j}<1$, then $\prod_{j=1}^{\infty}\left(1-t_{j}\right)$ converges if and only if $\sum_{j=1}^{\infty} t_{j}$ converges.

Proof: We refer to Apostol [5].

Theorem 74 Let the potential $\phi$ (which is defined in a neighborhood of the Julia set $J(f)$ ) satisfy the following inequality

$$
1-b_{n} \leq \frac{e^{\left[\phi\left(f_{a_{0} \cdots a_{n-1} l}(z)\right)\right]}}{e^{\left[\phi\left(f_{a_{0} \cdots a_{n-1} m}(z)\right)\right]}} \leq 1+b_{n},
$$

where $b_{n}$ is a sequence of real numbers with the property that $0<b_{n}<1$ and $b_{n} \leq \frac{c}{n^{p}}$ for some constants $c>0, p>1$. Then $\nu$ is a quasiinvariant measure on $\Sigma^{+}$.

Proof: First we show that the ratio of the image measure $\nu \circ \sigma^{-1}$ to the measure $\nu$ for a cylinder set $\left[a_{0} a_{1} \cdots a_{l-1}\right]$ is bounded by a constant $k$, i.e., there exists a constant $k$ such that

$$
\frac{\nu \circ \sigma^{-1}\left(\left[a_{0} a_{1} \cdots a_{l-1}\right]\right)}{\nu\left(\left[a_{0} a_{1} \cdots a_{l-1}\right]\right)} \leq k
$$

By using the definition of $\nu$ we have the following estimation for the numerator in (4.2):

$$
\begin{aligned}
& \nu \circ \sigma^{-1}\left(\left[a_{0} a_{1} \cdots a_{l-1}\right]\right) \\
\leq & \max _{j \in \mathcal{A}} p_{j} \cdot \sum_{i=1}^{d} P_{i, i a_{0}} P_{i a_{0}, i a_{0} a_{1}} \cdots P_{a_{0} a_{1} \cdots a_{l-2}, a_{0} a_{1} \cdots a_{l-1}} \\
\leq & \max _{j \in \mathcal{A}} p_{j} \cdot \sum_{i=1}^{d} \frac{1}{d\left(1-b_{1}\right)} \cdot \frac{1}{d\left(1-b_{2}\right)} \cdots \frac{1}{d\left(1-b_{l}\right)} \\
= & \max _{j \in \mathcal{A}} p_{j} \cdot \frac{1}{d^{l-1} \cdot\left(1-b_{1}\right) \cdot\left(1-b_{2}\right) \cdots\left(1-b_{l}\right)} \\
\leq & \max _{j \in \mathcal{A}} p_{j} \cdot \frac{1}{d^{l-1} \cdot \prod_{n=1}^{\infty}\left(1-b_{n}\right)} \quad(\text { by Lemma } 73)
\end{aligned}
$$


Next for the denominator we have the estimation

$$
\begin{aligned}
& \nu\left(\left[a_{0} a_{1} \cdots a_{l-1}\right]\right) \\
\geq & \min _{j \in \mathcal{A}} p_{j} \cdot\left(\frac{e^{\left[\phi\left(f_{a_{0} \cdots a_{l-1}}(z)\right)\right]}}{\sum_{i=1}^{d} e^{\left[\phi\left(f_{a_{0} \cdots a_{l-2}}(z)\right)\right]}}\right)^{l-1} \\
\geq & \min _{j \in \mathcal{A}} p_{j} \cdot\left(\frac{1}{d\left(1+b_{l-1}\right)}\right)^{l-1} \\
= & \min _{j \in \mathcal{A}} p_{j} \cdot\left(\frac{1}{d^{l-1}\left(1+b_{l-1}\right)^{l-1}}\right) .
\end{aligned}
$$

This minimum is above zero since $b_{(l-1)} \leq \frac{c}{(l-1)^{p}}$ and thus

$$
\begin{aligned}
\left(1+b_{(l-1)}\right)^{l-1} & \leq\left(1+\frac{c}{(l-1)^{p}}\right)^{l-1} \\
& \leq\left(1+\frac{c}{(l-1)}\right)^{l-1} \stackrel{l \rightarrow+\infty}{\longrightarrow} e^{c}<\infty
\end{aligned}
$$

We have thus shown inequality (4.2). We complete our proof by using an arbitrary measurable set $A \in \mathcal{B}$.

Let $\epsilon>0$. Choose $\delta=\epsilon / k$. Let $A \in \mathcal{B}$ with $\nu(A)<\epsilon / k$. Set $\epsilon_{1}=\epsilon / k-\nu(A)>0$. By regularity of $\nu$ there exists an open set $O \supset A$ such that

$$
\begin{aligned}
\nu(A)>\nu(O)-\epsilon_{1}=\nu(O)-\epsilon / k+\nu(A) \\
\Rightarrow \quad \nu(O)<\epsilon / k
\end{aligned}
$$

As the class of cylinder sets form a countable basis for the product topology on $\Sigma^{+}$and is a semi-algebra, we can express $O$ as a disjoint union of cylinder sets $C_{i}, \quad i \geq 1$ :

$$
O=\cup_{i} C_{i} \Rightarrow \nu\left(\cup_{i} C_{i}\right)<\epsilon / k
$$


Hence

$$
\begin{aligned}
\sigma^{*} \nu(A) & \leq \sigma^{*} \nu(O)=\sum_{i} \sigma^{*} \nu\left(C_{i}\right) \\
& \leq k \cdot \sum_{i} \nu\left(C_{i}\right)<k \cdot \epsilon / k=\epsilon \text { (by inequalities (4.2) and (4.3)). }
\end{aligned}
$$

Hence, by using Lemma 72 above, we get $\sigma^{*} \nu \ll \nu$. The other part of quasi-invariance $\nu \ll \sigma^{*} \nu$ is easily obtained in a similar calculation.

Lemma 75 Let the potential $\phi$ satisfy the same inequality as in Theorem 74. Then the measure $\nu$ is equivalent to a $(1 / d, 1 / d, \cdots, 1 / d)$ Bernoulli measure and hence ergodic.

Proof: The proof is similar to the above theorem.

The concept of the wandering set in dynamical systems and ergodic theory formalizes a certain idea of movement and mixing in such systems. When a dynamical system has a wandering set of non-zero measure, then the system is called dissipative. This is very much the opposite of a conservative system, for which the ideas of the Poincaré recurrence theorem apply. Intuitively, the connection between wandering sets and dissipation is easily understood: if a portion of the phase space "wanders away" during normal time-evolution of the system, and is never visited again, then the system is dissipative. A traditional question asked about quasi-invariant measures is whether they are dissipative or conservative. Recall that an ergodic measure is called conservative if it satisfies the Poincaré Recurrence Theorem and is called dissipative otherwise. Now we show that the measure $\nu$ is conservative:

Lemma 76 The measure $\nu$ is conservative.

Proof: Let $A \in \mathcal{B}\left(\Sigma^{+}\right)$have $\nu(A)>0$. We prove that for almost all points $x \in A$ the orbit $\left\{\sigma^{n} x\right\}_{n \geq 0}$ returns to $A$ infinitely often. Let 
$F=\left\{x \in A: \sigma^{n} x \notin A, \forall n \geq 1\right\}$; then it suffices to show that $\nu(F)=0$. Towards this end, we first observe that $\sigma^{-m} F \cap \sigma^{-n} F=\emptyset$ when $n>m$, say. If this were not the case and $w \in \sigma^{-m} F \cap \sigma^{-n} F$, then $\sigma^{m} w \in F$ and $\sigma^{n-m}\left(\sigma^{m} w\right) \in F \subset A$, which contradicts the definition of $F$. Thus since the sets $\left\{\sigma^{-n} F\right\}_{n \geq 0}$ are disjoint we see that for any $\sigma$-invariant measure $\mu$ we have

$$
\sum_{n=0}^{\infty} \mu\left(\sigma^{-n} F\right)=\mu\left(\cup_{n=0}^{\infty} \sigma^{-n} F\right) \leq \mu\left(\Sigma^{+}\right)=1
$$

and then, because $\mu$ is $\sigma$-invariant, $\mu(F)=\mu\left(\sigma^{-1} F\right)=\cdots=\mu\left(\sigma^{-n} F\right)=$ $\cdots$, we can only have $\mu(F)=0$. Since our measure $\nu$ is absolutely continuous with respect to the $\sigma$-invariant $(1 / d, 1 / d, \cdots, 1 / d)$ Bernoulli measure, we immediately have $\nu(F)=0$ as required.

\subsection{Gibbs and Equilibrium Measures}

Now we proceed to prove that $\nu$ is a Gibbs measure for a suitable potential on the space $\Sigma^{+}$. We need the notion of the Jacobian of the shift map with respect to a probability measure and a few other results.

Consider $x \in \Sigma^{+}$, so $x=x_{1} x_{2} \cdots x_{n} \cdots$. For $n \in \mathbb{N}$, define

$$
J_{n}(x)=\left\{\begin{array}{lc}
\mu\left(x_{2} \cdots x_{n}\right) / \mu\left(x_{1} \cdots x_{n}\right) & \text { if the denominator is nonzero } \\
+\infty & \text { otherwise. }
\end{array}\right.
$$

Note that the function $J_{n}$ is bounded below by 1 when $\mu$ is a shift invariant measure.

Lemma 77 The sequence $\left\langle J_{n}\right\rangle$ is an $L^{1}(\mu)$ martingale with respect to the filtration $\left(B_{n}\right)$, where $B_{n}$ is the $\sigma$-algebra generated by the set $\mathcal{A}_{n}$ of cylinders $\left[x_{1} \cdots x_{n}\right]$. 
Proof: Consider $x \in \mathcal{A}_{n}$, and assume $\mu\left(\left[x_{1} \cdots x_{n}\right]\right)>0$. On this cylinder, we have the conditional expectation

$$
\begin{aligned}
& E\left(J_{n+1} \mid B_{n}\right)\left(x_{1} x_{2} \cdots x_{n} \cdots\right)=\frac{1}{\mu\left(\left[x_{1} \cdots x_{n}\right]\right)} \int_{\left[x_{1} \cdots x_{n}\right]} J_{n+1} d \mu \\
= & \frac{1}{\mu\left(\left[x_{1} \cdots x_{n}\right]\right)} \sum_{x_{n+1} \in \mathcal{A}} \int_{\left[x_{1} \cdots x_{n} x_{n+1}\right]} J_{n+1} d \mu \\
= & \frac{1}{\mu\left(\left[x_{1} \cdots x_{n}\right]\right)} \sum_{x_{n+1} \in \mathcal{A}} \mu\left(\left[x_{1} \cdots x_{n} x_{n+1}\right]\right) \frac{\mu\left(\left[x_{2} \cdots x_{n} x_{n+1}\right]\right)}{\mu\left(\left[x_{1} \cdots x_{n} x_{n+1}\right]\right)} \\
= & J_{n}\left(x_{1} x_{2} \cdots x_{n} \cdots\right) .
\end{aligned}
$$

Furthermore $E\left(J_{n}\right)$, is finite, since

$$
\begin{aligned}
E\left(J_{n}\right) & =\int_{\Sigma^{+}} J_{n}(x) d \mu(x) \\
& =\int_{\cup \mathcal{A}_{n}} J_{n}(x) d \mu(x) \\
& =\sum_{\left[x_{1} \cdots x_{n}\right]} \frac{\mu\left(\left[x_{2} \cdots x_{n}\right]\right)}{\mu\left(\left[x_{1} \cdots x_{n}\right]\right)} \mu\left(\left[x_{1} \cdots x_{n}\right]\right) \\
& =\sum_{\left[x_{1} \cdots x_{n}\right]} \mu\left(\left[x_{2} \cdots x_{n}\right]\right) \\
& \leq d
\end{aligned}
$$

Corollary 78 The function $J_{\mu}(x)=\lim _{n \rightarrow \infty} J_{n}(x)$ exists almost everywhere. It is called the Jacobian of the shift map $\sigma$ with respect to the measure $\mu$.

Proof: The proof is a direct consequence of the martingale convergence theorem( see e.g. Resnick [51]).

We will see that the Jacobian of the shift map $\sigma$ with respect to the measure $\nu$ can be made Lipschitz continuous by imposing suitable 
conditions on the map $\phi$ which appears explicitly in $\nu$. Before doing that, we state the well-known Weierstrass M-test which will be used to prove our result. We also need the notion of infinite products. An introduction to the theory of infinite products can be found in Apostol [5].

\section{Theorem 79 Weierstrass M-test}

Let $\sum u_{n}(z)$ be an infinite series of one-valued functions of $z$ defined in a bounded, closed domain $D$ and let there exist a series $\sum M_{n}$ of positive constants independent of $z$ such that $\left|u_{n}(z)\right| \leq M_{n}$ for all $n \in D$ and $\sum M_{n}$ is convergent. Then the series $\sum u_{n}(z)$ is uniformly and absolutely convergent in the domain D.

Now we are ready to prove the following result which will be used to prove that the Jacobian of the shift map $\sigma$ with respect to our measure $\nu$ is Lipschitz continuous under a suitable condition on the map $\phi$.

Lemma 80 Let the potential $\phi$ (which is defined in a neighbourhood of the Julia set $J(f)$ ) satisfy the following inequality

$$
1-b_{n} \leq \frac{e^{\left[\phi\left(f_{a_{0} \cdots a_{n-1} l}(z)\right)\right]}}{e^{\left[\phi\left(f_{a_{0} \cdots a_{n-1} m}(z)\right)\right]}} \leq 1+b_{n},
$$

where $b_{n}$ is a sequence of real numbers with the property that $0<b_{n}<1$ and $b_{n} \leq \frac{c}{n^{p}}$ for some constants $c>0, p>1$. Then the Jacobian $J_{\nu}$ of the measure $\nu$, defined by

$$
J_{\nu}\left(x_{0} x_{1} \cdots\right):=\lim _{n \rightarrow \infty} J_{n}\left(x_{0} x_{1} \cdots\right):=\lim _{n \rightarrow \infty} \frac{\nu\left(\left[x_{1} \cdots x_{n}\right]\right)}{\nu\left(\left[x_{0} \cdots x_{n}\right]\right)},
$$

is a continuous map on $\Sigma^{+}$.

Proof: To prove the result we use the well-known fact that the limit $f$ of a uniformly convergent sequence $\left\{f_{n}\right\}$ of continuous functions on a topological space $X$ is a continuous function. 
Clearly, each $J_{n}$ is (uniformly) continuous on $\Sigma^{+}$. Now we have

$$
\begin{aligned}
J_{n}\left(x_{0} x_{1} \cdots\right) & :=\frac{\nu\left(\left[x_{1} \cdots x_{n}\right]\right)}{\nu\left(\left[x_{0} \cdots x_{n}\right]\right)} \\
& =\frac{1}{p_{x_{0}}} \times \frac{p_{x_{1}}}{P_{x_{0}, x_{0} x_{1}}} \times \frac{P_{x_{1}, x_{1} x_{2}}}{P_{x_{0} x_{1}, x_{0} x_{1} x_{2}}} \times \cdots \times \frac{P_{x_{1} \cdots x_{n-1}, x_{1} \cdots x_{n}}}{P_{x_{0} \cdots x_{n-1}, x_{0} \cdots x_{n}}}
\end{aligned}
$$

Set

$$
1+u_{n}(z)=\frac{P_{x_{1} \cdots x_{n-1}, x_{1} \cdots x_{n}}}{P_{x_{0} \cdots x_{n-1}, x_{0} \cdots x_{n}}}=\frac{e^{\phi\left(f_{x_{1} \cdots x_{n}}(z)\right)}}{\sum_{l=1}^{d} e^{\phi\left(f_{x_{1} \cdots x_{n-1} l}(z)\right)}} \times \frac{\sum_{l=1}^{d} e^{\phi\left(f_{x_{0} \cdots x_{n-1} l}(z)\right)}}{e^{\phi\left(f_{x_{0} \cdots x_{n}}(z)\right)}}
$$

With a little calculation we obtain that

$$
-\frac{b_{n}+b_{n-1}}{1+b_{n-1}}<u_{n}(z)<\frac{b_{n}+b_{n-1}}{1-b_{n-1}}
$$

which implies that $\left|u_{n}(z)\right|<\frac{b_{n}+b_{n-1}}{1-b_{n-1}}=: M_{n}$. Clearly, the series $\sum M_{n}$ is convergent.

Theorem 81 Let the potential $\phi$ be as above in Lemma 80 satisfying the same condition. Then the Jacobian of the shift map $\sigma$ with respect to the measure $\nu$ is Lipschitz continuous.

Proof: Let us consider $x=x_{0} \cdots x_{k-1} y, x^{\prime}=x_{0} \cdots x_{k-1} y^{\prime} \in \mathcal{W}$ Now for $n \geq k$,

$$
\begin{aligned}
\frac{J_{n}(x)}{J_{n}\left(x^{\prime}\right)} & =\frac{\nu\left(\left[x_{1} \cdots x_{k-1} y\right]\right)}{\nu\left(\left[x_{0} \cdots x_{k-1} y\right]\right)} \times \frac{\nu\left(\left[x_{0} \cdots x_{k-1} y^{\prime}\right]\right)}{\nu\left(\left[x_{1} \cdots x_{k-1} y^{\prime}\right]\right)} \\
& =\frac{\nu\left(\left[x_{1} \cdots x_{k-1} y\right]\right)}{\nu\left(\left[x_{1} \cdots x_{k-1} y^{\prime}\right]\right)} \times \frac{\nu\left(\left[x_{0} \cdots x_{k-1} y^{\prime}\right]\right)}{\nu\left(\left[x_{0} \cdots x_{k-1} y\right]\right)}
\end{aligned}
$$

Let us consider only the first term of the right side; the second term can be treated similarly. 


$$
\begin{aligned}
& \frac{\nu\left(\left[x_{1} \cdots x_{k-1} y\right]\right)}{\nu\left(\left[x_{1} \cdots x_{k-1} y^{\prime}\right]\right)} \\
= & \frac{p_{x_{1}} P_{x_{1}, x_{1} x_{2}} P_{x_{1} x_{2}, x_{1} x_{2} x_{3}} \cdots P_{x_{1} \cdots x_{k-1}, x_{1} \cdots x_{k-1} y}}{p_{x_{1}} P_{x_{1}, x_{1} x_{2}} P_{x_{1} x_{2}, x_{1} x_{2} x_{3}} \cdots P_{x_{1} \cdots x_{k-1}, x_{1} \cdots x_{k-1} y^{\prime}}} \\
= & \frac{P_{x_{1} \cdots x_{k-1}, x_{1} \cdots x_{k-1} y}}{P_{x_{1} \cdots x_{k-1}, x_{1} \cdots x_{k-1} y^{\prime}}} \\
= & \frac{\exp \left[\phi\left(f_{x_{1} \cdots x_{k-1} y}(z)\right)\right]}{\sum_{l=1}^{d} \exp \left[\phi\left(f_{x_{1} \cdots x_{k-1} l}(z)\right)\right]} \times \frac{\sum_{l=1}^{d} \exp \left[\phi\left(f_{x_{1} \cdots x_{k-1} l}(z)\right)\right]}{\exp \left[\phi\left(f_{x_{1} \cdots x_{k-1} y^{\prime}}(z)\right)\right]}
\end{aligned}
$$

By using the condition on $\phi$ we can easily obtain the following two inequalities for the first and second term on the right hand side of the above equality:

$$
\begin{aligned}
& \frac{1}{d\left(1+b_{k-1}\right)} \leq \frac{\exp \left[\phi\left(f_{x_{1} \cdots x_{k-1} y}(z)\right)\right]}{\sum_{l=1}^{d} \exp \left[\phi\left(f_{x_{1} \cdots x_{k-1} l}(z)\right)\right]} \leq \frac{1}{d\left(1-b_{k-1}\right)} \\
& d\left(1-b_{k-1}\right) \leq \frac{\sum_{l=1}^{d} \exp \left[\phi\left(f_{x_{1} \cdots x_{k-1} l}(z)\right)\right]}{\exp \left[\phi\left(f_{x_{1} \cdots x_{k-1} y^{\prime}}(z)\right)\right]} \leq d\left(1+b_{k-1}\right)
\end{aligned}
$$

Consequently, we have

$$
\frac{1-b_{k-1}}{1+b_{k-1}} \leq \frac{\nu\left(\left[x_{1} \cdots x_{k-1} y\right]\right)}{\nu\left(\left[x_{1} \cdots x_{k-1} y^{\prime}\right]\right)} \leq \frac{1+b_{k-1}}{1-b_{k-1}}
$$

Similarly for the second term we have

$$
\frac{1-b_{k}}{1+b_{k}} \leq \frac{\nu\left(\left[x_{0} \cdots x_{k-1} y^{\prime}\right]\right)}{\nu\left(\left[x_{0} \cdots x_{k-1} y\right]\right)} \leq \frac{1+b_{k}}{1-b_{k}}
$$

Hence

$$
\frac{\left(1-b_{k-1}\right)}{\left(1+b_{k-1}\right)} \cdot \frac{\left(1-b_{k}\right)}{\left(1+b_{k}\right)} \leq \frac{J_{n}(x)}{J_{n}\left(x^{\prime}\right)} \leq \frac{\left(1+b_{k-1}\right)}{\left(1-b_{k-1}\right)} \cdot \frac{\left(1+b_{k}\right)}{\left(1-b_{k}\right)}
$$

which implies that

$$
\left|\frac{J_{n}(x)}{J_{n}\left(x^{\prime}\right)}-1\right| \leq \frac{2\left(b_{k}+b_{k-1}\right)}{1-\left(b_{k}+b_{k-1}\right)}
$$


Since the sequence $<J_{n}\left(x^{\prime}\right)>$ is convergent, $\left|J_{n}\left(x^{\prime}\right)\right|$ is bounded, say by a constant $K$. Therefore, we have

$$
\begin{aligned}
\left|J_{n}(x)-J_{n}\left(x^{\prime}\right)\right| & \leq \frac{2\left(b_{k}+b_{k-1}\right)}{1-\left(b_{k}+b_{k-1}\right)} \cdot\left|J_{n}\left(x^{\prime}\right)\right| \\
& \leq \frac{2 K\left(b_{k}+b_{k-1}\right)}{1-\left(b_{k}+b_{k-1}\right)} \\
& \leq \frac{2 K\left(\frac{c}{k^{p}}+\frac{c}{(k-1)^{p}}\right)}{1-\frac{2 c}{(k-1)^{p}}} \\
& \leq 2 K \cdot \frac{\frac{2 c}{(k-1)^{p}}}{1-\frac{2 c}{(k-1)^{p}}} \\
& =2 K \cdot \frac{2 c}{(k-1)^{p}-2 c} \\
& \left.=\widetilde{K} \theta^{k}=\widetilde{K} \cdot d_{\left(x, x^{\prime}\right.}\right)
\end{aligned}
$$

where $2 K=\widetilde{K}$, and the value of $c$ is given by $c_{\theta}:=c=\frac{1}{2} \frac{\theta^{k}(k-1)^{p}}{1+\theta^{k}}$.

By using the above Theorem 81 and the fact that the composition of two Lipschitz continuous functions is also Lipschitz continuous, it follows that the function $\psi(x)=-\log \left(J_{\nu}(x)\right), x \in \Sigma^{+}$, belongs to the family $\mathcal{F}_{\theta}^{+}$.

We also need the following Lemma containing the formula for the change of variables:

Lemma 82 For every $g \in C\left(\Sigma^{+}\right)$and a quasi-invariant measure $\mu$ we have

$$
\sum_{i=1}^{d} \int_{\Sigma^{+}} g(i x) \frac{1}{J_{\mu}(i x)} d \mu(x)=\int_{\Sigma^{+}} g d \mu .
$$

Proof: Note first of all that $\sigma^{*}(g \mu) \ll \mu$. Indeed, if $\mu(E)=0$, then quasi-invariance of $\mu$ implies

$$
\left|\sigma^{*}(g \mu)(E)\right|=\left|g \mu\left(\sigma^{-1} E\right)\right|=\left|\int_{\sigma^{-1} E} g d \mu\right| \leq\|g\|_{\infty} \sigma^{*} \mu(E)=0 .
$$


From the Radon-Nikodym theorem we have $\sigma^{*}(g \mu)=\lambda \mu$, with $\lambda \in$ $L^{1}(\mu)$ defined by the formula

$$
\lambda(x)=\lim _{n \rightarrow \infty} \frac{\sigma^{*} g \mu\left(\left[x_{1} \cdots x_{n}\right]\right)}{\mu\left(\left[x_{1} \cdots x_{n}\right]\right)} \quad \mu \text {-almost everywhere }
$$

However, we also have

$$
\sigma^{*} g \mu\left(\left[x_{1} \cdots x_{n}\right]\right)=\sum_{i=1}^{d} \int_{\left[i x_{1} \cdots x_{n}\right]} g d \mu=\sum_{i=1}^{d} g(i x) \mu\left(\left[i x_{1} \cdots x_{n}\right]\right)+\epsilon_{n},
$$

with $\left|\epsilon_{n}\right| \leq \omega_{n+1}(g) \sigma^{*} \mu\left(\left[x_{1} \cdots x_{n}\right]\right)$.

Since $\sigma^{*} \mu$ is absolutely continuous with respect to $\mu$, it follows easily from here that

$$
\lambda(x)=\sum_{i=1}^{d} g(i x) \frac{1}{J_{\mu}(i x)} .
$$

We obtain the result from these observations and the identity

$$
\sigma^{*} g \mu\left(\Sigma^{+}\right)=1=\int_{\Sigma^{+}} d\left(\sigma^{*} g \mu\right)=\int_{\Sigma^{+}} \lambda d \mu
$$

Let us take $\psi(x)=-\log \left(J_{\mu}(x)\right)$, then

$$
\mathcal{L}_{\psi}(g)(x)=\sum_{i=1}^{d} g(i x) e^{-\log \left(J_{\mu}(i x)\right)}=\sum_{i=1}^{d} g(i x) \frac{1}{J_{\mu}(i x)} .
$$

Thus the equality in the above lemma becomes

$$
\begin{aligned}
& \int_{\Sigma^{+}} \mathcal{L}_{\psi}(g)(x) d \mu(x)=\int_{\Sigma^{+}} g d \mu \\
\Rightarrow & \int_{\Sigma^{+}} g d \mathcal{L}_{\psi}^{*} \mu(x)=\int_{\Sigma^{+}} g d \mu \\
\Rightarrow & \mathcal{L}_{\psi}^{*} \mu=\mu
\end{aligned}
$$

Thus we may paraphrase the change of variable formula by saying

$$
\mathcal{L}_{\psi}^{*} \mu=\mu \text {. }
$$


Now, it follows from Proposition 43 and Corollary 44 that our measure $\nu$ is a Gibbs measure (for a suitable potential $g(x)$ as described in the remark after Corollary 44). We state this as a theorem:

Theorem 83 The measure $\nu$ is a Gibbs measure for a suitable potential on $\Sigma^{+}$.

Corollary 84 The Gibbs measure $\nu$, the $(1 / d, 1 / d, \cdots, 1 / d)$ Bernoulli measure, and the image measure of the measure of maximal entropy under the homeomorphism $\Phi$ are equivalent.

Proof: Follows from Corollary 62, Theorem 69 and Lemma 75.

Now let us consider the quasi-invariant measure $\nu$ and $\psi=-\log \left(J_{\nu}\right)$. We have seen that $\psi$ is Lipschitz. The change of variable formula shows that $\mathcal{L}_{\psi}^{*}(\nu)=\nu$. Let $\beta>0$ and $h>0$ be the eigenvalue and eigenfunction, respectively, of the operator $\mathcal{L}_{\psi}$ guaranteed by the Ruelle-PerronFrobenius Theorem 35; that is, $\mathcal{L}_{\psi} h=\beta h$ with $\nu(h)=1$. Then we have

$$
\begin{aligned}
& \mathcal{L}_{\psi}^{*} \nu(f)=\nu(f) \text { for all } f \in C\left(\Sigma^{+}\right) \\
\Rightarrow & \nu\left(\mathcal{L}_{\psi} f\right)=\nu(f) \text { for all } f \in C\left(\Sigma^{+}\right) \\
\Rightarrow & \nu\left(\mathcal{L}_{\psi} h\right)=\nu(h) \\
\Rightarrow & \nu(\beta h)=\nu(h) \\
\Rightarrow & \beta=1
\end{aligned}
$$

From Corollary 39 we know that $P(\psi)=\log \beta$, therefore $P(\psi)=$ $\log \beta=0$. Consequently, we have the following result:

Corollary 85 The measure $\gamma=h \nu$ is the unique $\sigma$-invariant probability measure with the property that

$$
P(\psi)=h_{\gamma}(\sigma)+\int_{\Sigma^{+}} \psi d \gamma=0 .
$$




\subsection{Capacity of the Julia Set and Harmonic Mea- sures}

Let $E$ be a bounded closed set in the $z$-plane and let $\mu$ be a positive mass distribution on $E$ of total mass 1, and set

$$
v=\inf _{\mu(E)=1} \int_{E} \int_{E} \log \frac{1}{|z-\zeta|} d \mu(z) d \mu(\zeta)
$$

When $c(E)>0$ (i.e. $v<+\infty$ ), by Theorem 25 there exists a unique positive mass distribution $\mu^{*}$ on $\mathrm{E}$ of total mass 1 such that

$$
v=\int_{E} \int_{E} \log \frac{1}{|z-\zeta|} d \mu^{*}(z) d \mu^{*}(\zeta)
$$

We call $\mu^{*}$ the equilibrium distribution of $E$. The supp $\mu^{*}$ is defined as the set of $a \in E$, such that any small neighborhood $U$ of $a$ contains a positive $\mu^{*}$-mass. supp $\mu^{*}$ is a closed subset of $E$.

Lemma 86 Let

$$
g(z)=\frac{a z+b}{c z+d}(a d-b c \neq 0)
$$

be a Möbius transformation, $F_{1}=g(F), F$ and $F_{1}$ be closed and bounded sets. Then $c(F)=0$ iff $c\left(F_{1}\right)=0$.

Proof: We only need to prove $c(F)=0 \Rightarrow c\left(F_{1}\right)=0$. Let $\mu$ be any positive mass distribution on $F_{1}$ of total mass 1 . Then $\sigma=\mu \circ g$ is a distribution on $F$ with unit mass and

$$
\begin{aligned}
& \int_{F_{1}} \int_{F_{1}} \log \frac{1}{|z-\zeta|} d \mu(z) d \mu(\zeta) \\
= & \int_{F} \int_{F} \log \frac{1}{|g(z)-g(\zeta)|} d \sigma(z) d \sigma(\zeta) \\
= & \int_{F} \int_{F} \log \frac{1}{|z-\zeta|} d \sigma(z) d \sigma(\zeta)-\log |a d-b c| \\
& \quad+\int_{F} \int_{F} \log |(c z+d)(c \zeta+d)| d \sigma(z) d \sigma(\zeta)
\end{aligned}
$$


Since $g(F)=F_{1}, g(z)=\frac{a z+b}{c z+d}$, the sets $F_{1}$ and $F$ are compact subsets of $\mathbb{C}$. Hence, there exists a positive number $A$ such that $|c z+d| \geq A>0$, for any $z \in F$. Therefore,

$$
\begin{aligned}
& \int_{F_{1}} \int_{F_{1}} \log \frac{1}{|z-\zeta|} d \mu(z) d \mu(\zeta) \\
\geq & \int_{F} \int_{F} \log \frac{1}{|z-\zeta|} d \sigma(z) d \sigma(\zeta)-\log |a d-b c|+2 \log A .
\end{aligned}
$$

Since $c(F)=0$, by definition,

$$
\int_{F} \int_{F} \log \frac{1}{|z-\zeta|} d \sigma(z) d \sigma(\zeta)=+\infty,
$$

and hence

$$
\int_{F_{1}} \int_{F_{1}} \log \frac{1}{|z-\zeta|} d \mu(z) d \mu(\zeta)=+\infty,
$$

for any $\mu \geq 0, \mu\left(F_{1}\right)=1$, that is $c\left(F_{1}\right)=0$.

Lemma 87 Let $T: \mathbb{C}_{\infty} \rightarrow \mathbb{C}_{\infty}$ be a rational function with deg $T \geq 2$ and $J$ be the Julia set of $T$. If $J$ is totally disconnected, then the Fatou set $J^{c}=\mathbb{C}_{\infty}-J$ cannot be parabolic.

Proof: $J^{c}=U$ is a Sullivan domain for $T U=U$. By Theorem 7.7 of Blanchard [9], if $U$ is a parabolic domain, then there exists a fixed point $P$ on $\partial U$, and it holds $T^{k}(z) \rightarrow P$ with $k \rightarrow \infty$ for all $z \in U$ and $T^{\prime}(P)=1$. By Theorem 3.10 of Blanchard [9], there are a local homeomorphism $h$ and an integer $k>1$, such that $h(P)=0$ and $h \circ T \circ h^{-1}(z)=z\left(1+z^{k}\right)=R(z)$. For $R(z)$, there are $k$ petal domains and $k$ rays. For any point on the rays, $R^{n}(z) \nrightarrow 0(z \neq 0)$. Since $h$ is a homeomorphism and $J$ is totally disconnected, we have a $z_{0} \in U$ such that $h\left(z_{0}\right)$ belongs to these rays. From $h \circ T \circ h^{-1}(z)=R(z)$, we know $h\left(T^{n}\left(z_{0}\right)\right)=R^{n}\left(h\left(z_{0}\right)\right)$. Since $T^{n}\left(z_{0}\right) \rightarrow P$, we have $h\left(T^{n}\left(z_{0}\right)\right) \rightarrow 0$. But $R^{n}\left(h\left(z_{0}\right)\right) \not 0$ is a contradiction. It follows that $U$ cannot be a parabolic domain. 
Lemma 88 Let $R(z)=\frac{z^{n}+a_{1} z^{n-1}+\cdots+a_{n}}{b_{1} z^{n-1}+\cdots+b_{n}}, n \geq 2,\left|b_{1}\right|<1, J$ be the Julia set and totally disconnected, then $c(J)>0$.

Proof: We refer to Yongcheng [58].

Theorem 89 Let $T: \mathbb{C}_{\infty} \rightarrow \mathbb{C}_{\infty}$ be a rational function with deg $T=$ $n \geq 2$ whose Julia set $J$ is bounded. Then $c(J)>0$.

Proof: If $J$ is not totally disconnected, $J$ has a component $E$ which is a continuum, and by Lemma 24, $c(E)>0$. Hence $c(J) \geq c(E)>0$.

If $J$ is totally disconnected, $U=J^{c}$ is connected, $T U=U$, and by Blanchard [9] (page 116 ), $U$ is a Sullivan domain for which five kinds of dynamics are possible:

1. $U$ is an attractive domain;

2. $U$ is a superattractive domain;

3. $U$ is a parabolic domain;

4. $U$ is a Siegel disk;

5. $U$ is a Herman ring.

Since $J$ is totally disconnected, (4) and (5) are not possible. By Lemma 87, (3) is not possible. So $U$ is an attractive or even superattractive domain. There exists $p \in U$ such that $T(p)=p$ and $\left|T^{\prime}(p)\right|<1$. Let

$$
g(z)=\frac{1}{z-p}(\text { if } p=\infty, g(z)=z)
$$

Then

$$
R(z)=\frac{z^{n}+a_{1} z^{n-1}+\cdots+a_{n}}{b_{1} z^{n-1}+\cdots+b_{n}} .
$$

$\infty$ is an attractive (or superattractive) fixed point of $R(z)$, so $\left|b_{1}\right|<$ 1. $J_{1}=g(J)$ is the Julia set of $R(z)$. From Lemma 88, we get $c\left(J_{1}\right)>0$. Hence by Lemma 86, $c(J)>0$. 
As a direct consequence of Theorem 89 and Theorem 33 we obtain the following result in our case:

Theorem 90 Let $T: \mathbb{C}_{\infty} \rightarrow \mathbb{C}_{\infty}$ be a rational map with deg $T=n \geq 2$ whose Julia set $J$ is bounded. Then its equilibrium measure $\mu$ is given by

$$
\mu=\omega_{D}(\infty, \cdot)
$$

where $D$ is the component of $\mathbb{C}_{\infty} \backslash J$ containing $\infty$.

In our case the logarithmic potential for the equilibrium measure turns out to be constant; this follows from the following theorem:

Theorem 91 Let $K$ be a compact set containing the bounded Julia set $J$ of a rational map $T: \mathbb{C}_{\infty} \rightarrow \mathbb{C}_{\infty}$ with deg $T=n \geq 2$. Let $\mu$ be the equilibrium measure for $K$. Then $p_{\mu}(z)=v(K)$ for all $z \in$ int $K$, where int $K$ denotes the interior of $K$.

Proof: Let $a \in$ int $K$ and let $B=B(a ; r)$ such that $\bar{B} \subseteq$ int $K$. Since the logarithmic potential is superharmonic, and by using Frostman's Theorem 27, we have

$$
\left(\pi r^{2}\right)^{-1} \int_{B} p_{\mu} d A \leq p_{\mu}(a) \leq v
$$

But, again from Frostman's Theorem, we know that $p_{\mu}(z)=v=$ $v(K)$ quasi-everywhere. Since quasi-everywhere implies almost-everywhere we immediately obtain that

$$
p_{\mu}(z)=v \text { a.e. } \Rightarrow\left(\pi r^{2}\right)^{-1} \int_{B} p_{\mu} d A=\left(\pi r^{2}\right)^{-1} \cdot v \cdot A(B)=v .
$$

Hence $p_{\mu}(a)=v$ as required.

Theorem 92 (Dirichlet Problem) Let $T: \mathbb{C}_{\infty} \rightarrow \mathbb{C}_{\infty}$ be a rational function with deg $T=n \geq 2$ whose Julia set $J$ is bounded. Then the Dirichlet Problem for the domain $U:=\mathbb{C}_{\infty} \backslash J$ has a unique solution.

Proof: The proof follows from Theorem 89, Theorem 91, Theorem 32, and Theorem 31. 


\subsection{An Example with a Rational Map}

In this last section we will look at an example map to illustrate the concept. Examples of maps with totally disconnected Julia sets include polynomials $z^{2}+c$ for $c \in \mathbb{C}$ large enough. We will see, in this section, that there are degree- 2 rational maps, not conjugate to polynomials, whose Julia set have the same features as $z^{2}+c$. This is summarized in the following:

Theorem 93 There exists a one-parameter family of degree-2 rational maps, which are hyperbolic, have a totally disconnected Julia set, and are not conjugate to a polynomial.

Proof: Let us consider the one-parameter family of degree-2 rational maps given by $f_{a}: \mathbb{C}_{\infty} \rightarrow \mathbb{C}_{\infty}, z \mapsto 1+a z-\frac{1}{z}$, where $a>1$.

Fixed Points of $f_{a}$ : Solving $f_{a}(z)=z$ we obtain

$$
z=\frac{-1 \pm \sqrt{1+4(a-1)}}{2(a-1)} .
$$

Clearly, both the fixed points, say $z_{1}$ and $z_{2}$, are real since $a>1$, and $z=\infty$ is also a fixed point. As $f_{a}$ is a rational map of degree $d=2$, these $d+1=3$ points constitute the set of the function's fixed points. Since the first derivative $f_{a}^{\prime}(z)=a+\frac{1}{z^{2}}$, and $z_{1}$ and $z_{2}$ are real, it follows that $f_{a}^{\prime}\left(z_{1}\right)>a>1$ and $f_{a}^{\prime}\left(z_{2}\right)>a>1$. So $z_{1}, z_{2}$ are repelling fixed points of $f_{a}$ belonging to the Julia set $J\left(f_{a}\right)$. It is easy to calculate that

$$
\begin{aligned}
F(z):=g \circ f_{a} \circ g^{-1} & =\frac{z}{a+z-z^{2}}, \text { where } g: z \mapsto \frac{1}{z}, \\
F^{\prime}(z) & =\frac{a+z^{2}}{\left(a+z-z^{2}\right)^{2}}
\end{aligned}
$$

and hence $F^{\prime}(0)=\frac{1}{a}<1$. So, $z=\infty$ is an attracting fixed point of $f_{a}$, which belongs to the Fatou set $F\left(f_{a}\right)$.

Critical Points of $f_{a}$ : Setting $f_{a}^{\prime}(z)=a+\frac{1}{z^{2}}=0$ we obtain that $z= \pm \frac{i}{\sqrt{a}}$ are the critical points of $f_{a}$. Now we wish to find out whether $J \cap P\left(f_{a}\right)=\emptyset$. 
We recall that the postcritical set $P\left(f_{a}\right)$ is the closure of the forward orbits of the critical points of $f_{a}$ :

$$
P\left(f_{a}\right)=\overline{\bigcup_{n>0, f_{a}^{\prime}(c)=0} f_{a}^{n}(c) .}
$$

We will show in two ways that the map $f_{a}$ is hyperbolic, analytically and numerically (by using Matlab).

Analytic Proof: The analytic proof follows easily from the wellknown Fatou's Theorem which states:

Every attracting cycle for a polynomial or rational function attracts at least one critical point.

We have seen that there are three fixed points - two, $z_{1}$ and $z_{2}$, are repelling (and belong to the Julia set) and the point of infinity $\infty$ is the only attracting fixed point (which gives rise to an attracting cycle of one point). So, by Fatou's Theorem, it immediately follows that the orbit of one of the two critical points of the rational map $f_{a}$, say $z=+\frac{i}{\sqrt{a}}$, converges to the point at infinity. Since the points of the orbit of the other critical point $z=-\frac{i}{\sqrt{a}}$ are simply the complex conjugate of the points of the orbit of $z=+\frac{i}{\sqrt{a}}$, it follows that the orbit of the critical point $z=-\frac{i}{\sqrt{a}}$ also converges to the point at infinity.

Numerical Proof: Now we use Matlab to compute the orbits of the critical point $z=+\frac{i}{\sqrt{a}}$ for $f_{a}$. The orbit of the other critical point generates simply the conjugate numbers of the orbits of $z=+\frac{i}{\sqrt{a}}$.

The following Matlab script asks the user to provide the parameter value $a$ and the number of points in the orbit. After providing these input values, the script calculates the orbit and plots the absolute values of these points which help to see the pattern.

$\%$ As our map f_a and the critical point depend on the parameter a, let us $\%$ use Matlab's symbolic math for the calculations.

syms a $z$

$\%$ Enter the rational map 


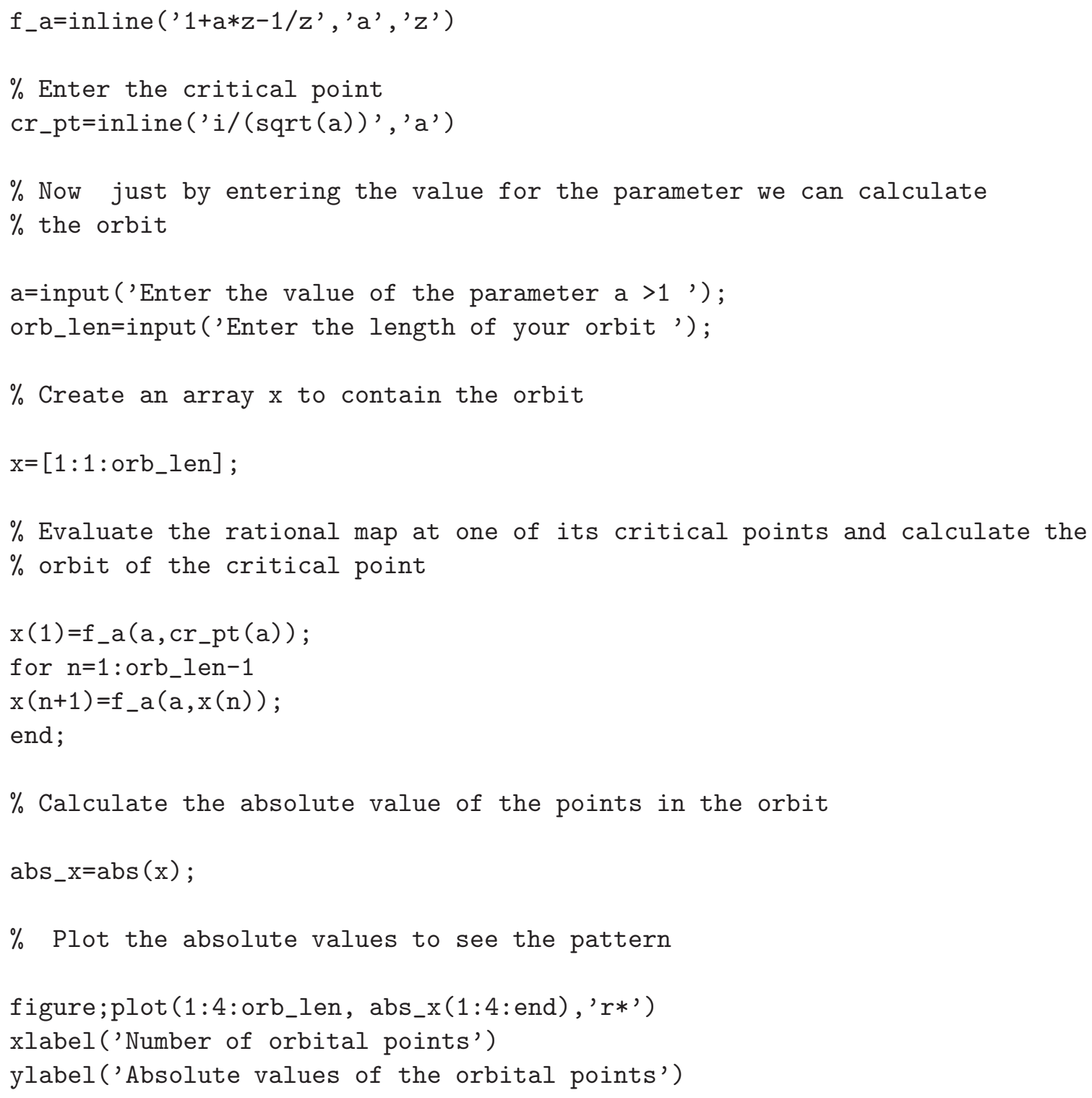

Here is a typical output of the script for parameter value $a=2$ and orbit length 500:

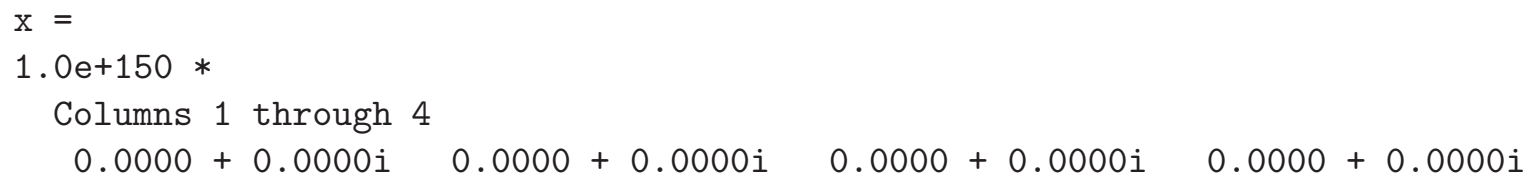



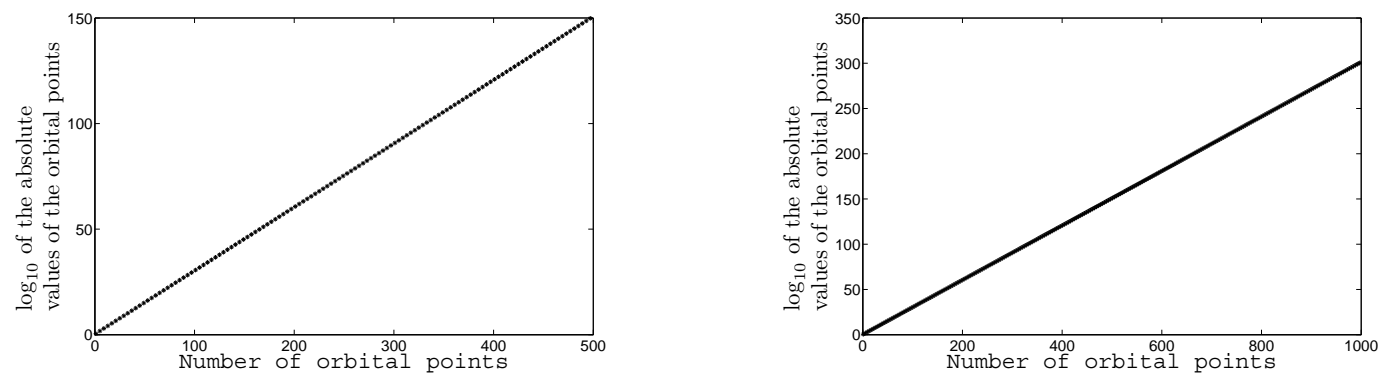

Figure 4.1: $\log _{10}$ of the absolute values of 500 (left graph) and 1000 (right graph) orbital points versus the number of orbital points

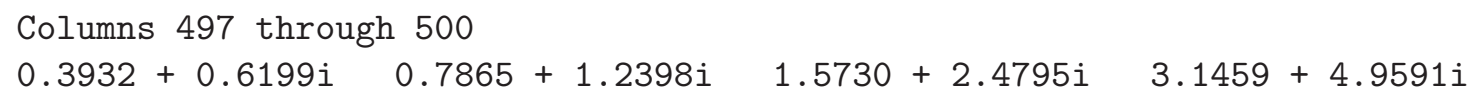
0.7341
1.4682
2.9364
5.8727

The example uses a rather small number of 500 points which still give us a clear pattern of increasing absolute values of the orbital points. The same pattern appears for 1000 points as shown in the above figure on the right which confirms that the points tend to infinity.

As $z=\infty \in F\left(f_{a}\right)$, we conclude that $J\left(f_{a}\right) \cap P\left(f_{a}\right)=\emptyset$. In other words, $f_{a}$ is hyperbolic. Now we proceed to determine whether the Julia set of the map $f_{a}$ is bounded and totally disconnected.

The boundedness of the Julia set $J\left(f_{a}\right)$ is immediate since $\infty \in F\left(f_{a}\right)$ and $J\left(f_{a}\right) \cap F\left(f_{a}\right)=\emptyset$.

The total disconnectedness of the Julia set follows from the following theorem:

Theorem 94 Let $f$ be a rational map of degree $d$, where $d \geq 2$, and let $\zeta$ be a (super)attracting fixed point of $f$. If all of the critical points 
of $f$ lie in the immediate attracting basin of $\zeta$, then $J(f)$ is a Cantor set.

Proof: See Beardon [7]: Theorem 9.8.1, P 227.

Is $f_{a}$ conjugate to a polynomial? Theorem 2.4.1 in Beardon [7] states that a non-constant rational map $R$ is conjugate to a polynomial if and only if there is some $w$ in $\mathbb{C}_{\infty}$ with $R^{-1}\{w\}=\{w\}$.

For $z \in \mathbb{C}$ we know that the points $z_{1}$ and $z_{2}$ are the only fixed points of $f_{a}$. It can easily be seen that $f_{a}^{-1}\left(z_{1}\right) \neq\left\{z_{1}\right\}$ and $f_{a}^{-1}\left(z_{2}\right) \neq\left\{z_{2}\right\}$. For $z=\infty$ we have $f_{a}^{-1}(\infty)=\{0, \infty\}$. Hence $f_{a}$ is not conjugate to a polynomial.

Now we analyze the map $f_{a}$ in order to locate its Julia set and get more information on the formation of the Julia set. After expressing the map as

$$
f_{a}(z)=1+a z-1 / z=\left[1+a x-x /\left(x^{2}+y^{2}\right)\right]+i * y\left[a+1 /\left(x^{2}+y^{2}\right)\right],
$$

we see that the real line is mapped onto itself and the preimage of a point in the real line also belongs to the real line. That is, the real line in union with infinity $\mathbb{R} \cup\{\infty\}$ is completely invariant under the map $f_{a}$, and hence, by minimality of the Julia set as a complete invariant set under $f_{a}$, implies that $J$ is contained in the real line. By restricting $f_{a}$ on $\mathbb{R}$ we can visualize the map's dynamics (see the graph of $f_{a}$ in Figure 4.2 below) which we will use in the following derivation showing that the Julia set is totally disconnected.

Now we prove that the whole half plane $\mathcal{R} e(z)>1$ is contained in the attracting basin of infinity. Indeed, if $z=x+i y$ with $x>1$, then from $f_{a}(z)=1+a x-x /\left(x^{2}+y^{2}\right)+i \star\left(a y+y /\left(x^{2}+y^{2}\right)\right)$ we get that the real part of $f_{a}(z)$ is $1+a x-x /\left(x^{2}+y^{2}\right)>1+a x-1 / x>a x>x$. Iterating, we see that the orbit of a point in the half plane $\operatorname{Re} e(z)>1$ tends to $\infty$, since the real part tends to $\infty$. In particular, the interval $(1, \infty)$ is in the Fatou set.

As and aside, we also observe easily that the upper half plane $(\mathcal{I} m(z)=$ $y>0)$ and lower half plane $(\mathcal{I} m(z)=y<0)$ are mapped into them- 


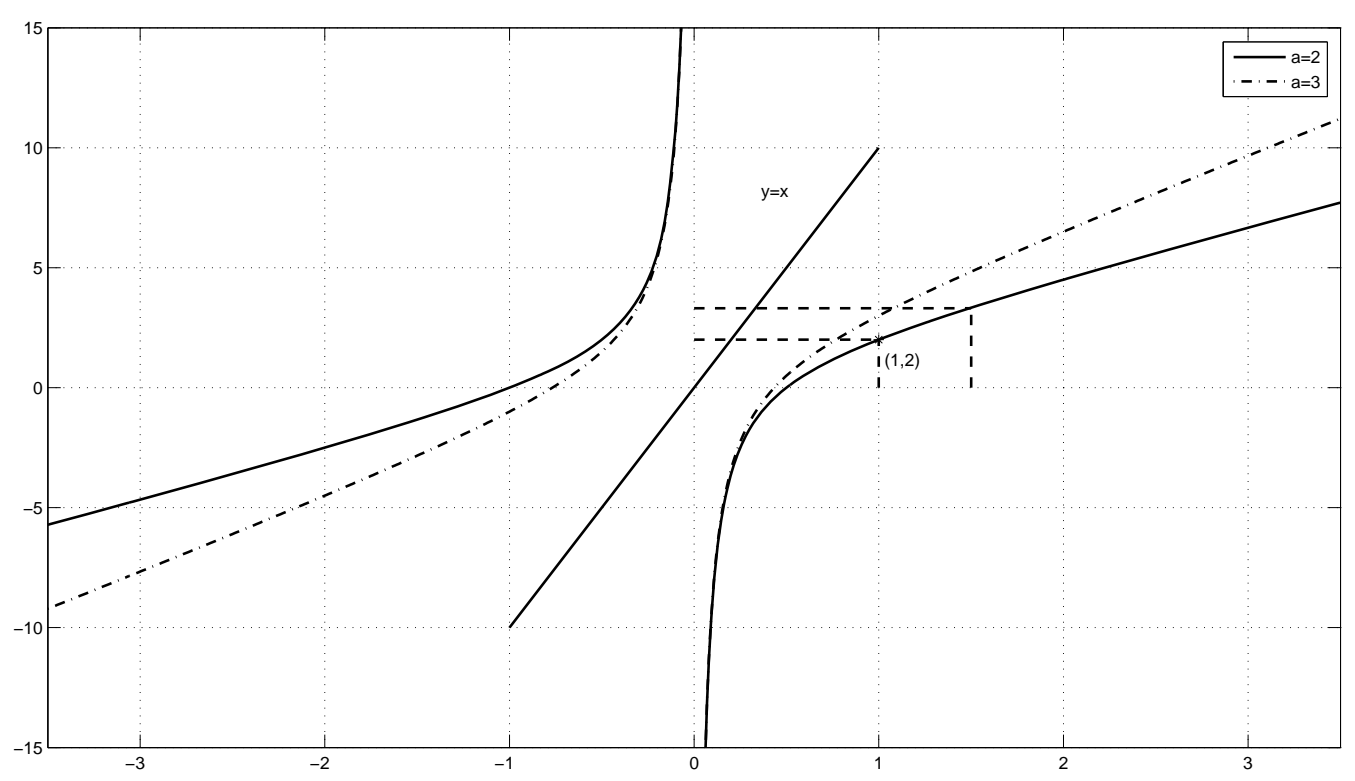

Figure 4.2: Graph of $f_{a}(a, x)=1+a x-\frac{1}{x}$

selves.

It is easy to see from the graph of $f_{a}$ that the interval $(2, \infty)$ has two preimages - one is the interval $(1, \infty)$ and the other interval is $\left(-\frac{1}{2}, 0\right)$ on the negative real line. These two intervals $(1, \infty)$ and $\left(-\frac{1}{2}, 0\right)$ each have again two preimages; each of these preimages has again two preimages - one on the negative real line and the other one in the positive real line and so on. Once we remove all those preimages, what remains is the Cantor Julia set.

Finally, we briefly point out how several results in Ransford [50] and Yongcheng [58] concerning the measure of equilibrium, harmonic measure and capacity can be discussed for such an example map.

Let us find a Möbius map which transforms the set $\mathbb{R} \cup\{\infty\}$ into a unit circle in the complex plane $\mathbb{C}$. This map is easily obtained by selecting three points on the extended real line, say $0,1, \infty$, which should be mapped to three non-collinear points on the circle say $1, i,-1$. Now 


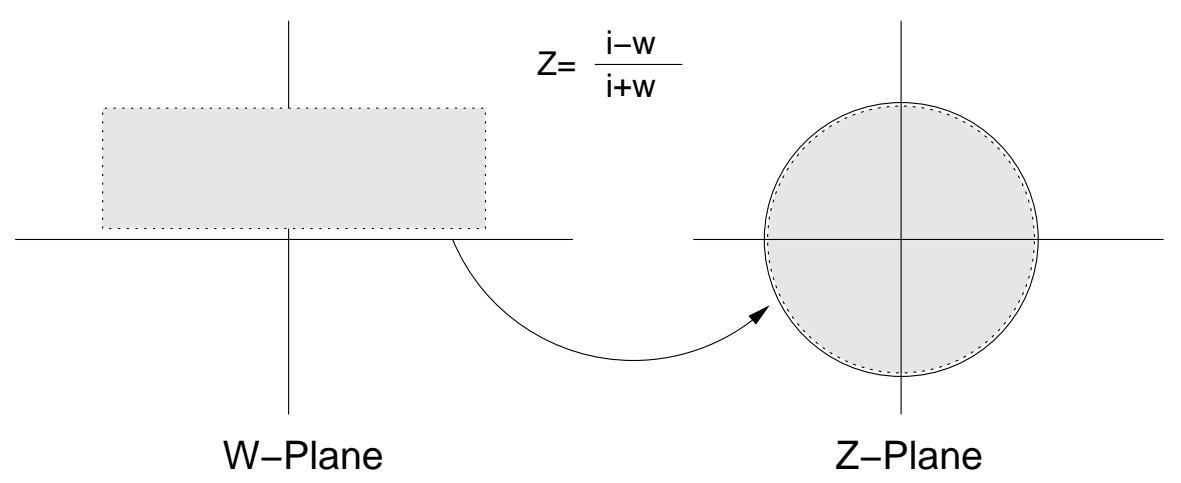

Figure 4.3: Möbius map $z=\frac{i-w}{i+w}$

we compute this map:

Let $z$ be mapped onto $w$ and since a Möbius map preserves a crossratio we have

$$
\begin{aligned}
& \frac{(w-0)(1-\infty)}{(0-1)(\infty-w)}=\frac{(z-1)(i+1)}{(1-i)(-1-z)} \\
\Rightarrow & z=\frac{i-w}{i+w}
\end{aligned}
$$

Writing $w=u+i v$ the transformation can be written as

$$
z=\frac{i-w}{i+w}=\frac{i-(u+i v)}{i+(u+i v)}=\frac{u+i(1-v)}{u+i(1+v)}
$$

Therefore $|z|=\frac{u^{2}+(1-v)^{2}}{u^{2}+(1+v)^{2}}$. Clearly $|z|=1$ when $v=0$, i.e. the real axis in the $w$-plane is mapped into the circle $|z|=1$.

By applying such a Möbius map $g$ on $\mathbb{R} \cup\{\infty\}$ we see that the image of the Julia set $J\left(f_{a}\right)$, i.e. $g\left(J\left(f_{a}\right)\right)$, will be contained in the circle $|z|=1$. We know from a theorem in Beardon (Theorem 3.1.4, on page 50) that $g\left(J\left(f_{a}\right)\right)=J\left(g \circ f_{a} \circ g^{-1}\right)$. Since $g \circ f_{a} \circ g^{-1}$ is a rational map of degree 2 with a bounded Julia set $J\left(g \circ f_{a} \circ g^{-1}\right)$, the capacity $c\left(J\left(g \circ f_{a} \circ g^{-1}\right)\right)=c\left(g\left(J\left(f_{a}\right)\right)\right)>0$. We also note that $g\left(J\left(f_{a}\right)\right)$ is totally disconnected since $g$ is a homeomorphism, and total disconnectedness is a topological property. 
Since $g\left(J\left(f_{a}\right)\right)$ is compact and non-polar, several results in Ransford [50] and Yongcheng [58] related to the measure of equilibrium, to the harmonic measure, and to capacity can be derived. Further, by establishing certain relations between measures such as absolute continuity or equality of the measures on $g\left(J\left(f_{a}\right)\right)$, and since $g: J\left(f_{a}\right) \rightarrow$ $g\left(J\left(f_{a}\right)\right)$ is a homeomorphism, we can draw conclusions similar to those in Ransford [50] and Yongcheng [58] for the corresponding measures on $J\left(f_{a}\right)$. 


\section{Bibliography}

[1] Aggarwal, R.S.: Topology, S. Chand \& Company (Pvt) Ltd., New Delhi, 1989.

[2] Ahlfors, L.V.: Complex Analysis (third edition), McGraw-Hill, 1979.

[3] Alexander, D. A History of Complex Dynamics. F. Vieweg \& Sohn, Braunshweig/Wiesbaden, 1994.

[4] Aliprantis, C.D., Burkinshaw, O.: Principles of Real Analysis, Academic Press, London, 1998.

[5] Apostol, T. M.: Mathematical Analysis, Reading (Mass.), 1974.

[6] Barlow, M.T. and Perkins, E.A.: Brownian motion on the Sierpi'nski gasket, Probab. Th. Rel. Fields 79 (1988), 543-624.

[7] Beardon, A.F.: Iteration of Rational Functions, Springer-Verlag New york, Inc. 1991.

[8] Billingsley, P.: Ergodic theory and information, John Wiley and Sons, New york, 1965.

[9] Blanchard, P.: Complex analytic dynamics on the Riemann sphere, Bull. Amer. Math. Soc., 11, (1984), 85-141.

[10] Bowen, R.: Equilibrium States and the Ergodic Theory of Anosov Diffeomorphisms, Springer-Verlag Berlin, Heidelberg, 1975. 
[11] Brolin, H.: Invariant sets under iteration of rational functions, Ark. Mat. 6, 103-144, 1975.

[12] Carlson, L. and Gamelin, T.: Complex Dynamics. Universitext, Springer-Verlag, 1993.

[13] Chung, K. L.: Markov Chains With Stationary Transition Probabilities, Springer-Verlag Berlin, 1967.

[14] Cohn, D.L.: Measure Theory, Birkhäuser Boston, 1980.

[15] Conway, J.B.: Functions of One Complex Variable II, SpringerVerlag, New York, 1995.

[16] Denker, M., Sato, S.: Sierpiński Gasket as a Martin Boundary, Potential Analysis 14(3): 211-232, May 2001.

[17] Denker, M., Sato, S.: Sierpiński Gasket as a Martin Boundary II, Potential Analysis 14(3): 211-232, May 2001.

[18] Denker, M., Koch, S.: A Poisson formula for harmonic functions on the Sierpiński Gasket, Forum Math. 12 (2000), 435-448.

[19] Denker, M., Urbański, M.: Ergodic theory of equilibrium states for rational maps, Nonlinearity 4 (1991) 103-134.

[20] Denker, M., Urbański, M.: On the existence of conformal measures, Transactions of the American Mathematical Society, Volume 328, Number 2, 1991.

[21] Doob, J.L.: Discrete potential theory and boundaries, J. Math. Mech. 8 (1959), 433-458.

[22] Dynkin, E.B.: Boundary theory of Markov processes(the discrete case), Russian Math. Surveys 24 (1969), 1-42.

[23] Eremenko, A.E., Lyubich, M. Yu.: The dynamics of analytic transformations, Leningrad Math. J., Vol 1(1990), No. 3. 
[24] Falconer, K.J.: Techniques in Fractal Geometry, Wiley, 1997.

[25] Fatou, P.: Sur les equations fonctionelles, Bulletin de la Sociétémathématique de France, 47 (1919), 161-271.

[26] Fatou, P. Sur les equations fonctionelles. Bulletin de la Sociétémathématique de France 48(1920), 33-94.

[27] Fatou, P. Sur les equations fonctionelles. Bulletin de la Sociétémathématique de France 48(1920), 208-314.

[28] Furstenberg, H.: Random walks and discrete subgroups of Lie groups. Advances in Probab. Ed.: P. Ney, Vol. 1, 1-63. M. Dekker 1971.

[29] Goldstein, S.: Random walks and diffusion on fractals, In: H. Kesten (ed.), Percolation Theory and Ergodic Theory of Infinite Particle Systems, IMA Math. Appl. 8, Springer, New York, 1987, 121-129.

[30] Gottschalk, W.H., Hedlund, G.: Topological dynamics, Amer. Math. Coll. Publ. 36 Providence. R.I. 1955.

[31] Hubbard, J. Preface. In Tan Lei, editor, The Mandelbrot Set, Theme and Variations, pages xiii-xx. Cambridge University Press, 2000 .

[32] Julia, G.: Memoir sur l'iteration des fonctions rationnelles, J. Math. Pures Appll., 8 (1918), 47-245.

[33] Kigami, J.: A harmonic calculus on the Sierpiński spaces, Japan J. Appl. Math. 6 (1989), 259-290.

[34] Kigami, J.: Harmonic calculus on the p.c.f self-similar sets. Trans. Amer. Math. Soc. 335 (1993), 721-755.

[35] Kigami, J.: Analysis on fractals. Cambridge University Press, Cambridge, 2001. 
[36] Kusuoka, S.: A diffusion process on a fractal, In: K. Ito and N. Ikeda (eds.), Probabilistic Methods in Mathematical Physics, Taniguchi Symposium, Katata 1985, Kinokuniya, North Holland, 1987, 251-274.

[37] Kaimanovich, V.A., Versik, A.M.: Random walks on discrete groups: Boundary and entropy, Ann. Probab. 11 (1983), 457-490.

[38] A. Lopes: Equilibrium measures for rational maps. Ergodic Theory \& Dynamical Systems. 1986, 6, 393-399.

[39] Lyubich, M., Volberg, A. A comparison of harmonic and maximal measures for rational functions. Approximation by solutions of partial differential equations (Hanstholm, 1991), 127-139, NATO Adv. Sci. Inst. Ser.C Math. Phys. Sci., 365, Kluwer Acad. Publ., Dordrecht, 1992.

[40] McMullen, C.T.: Complex dynamics and renormalization, Princeton, NJ : Princeton Univ. Press, 1994.

[41] Mandelbrot, B.B.: Fractals: Form, Chance and dimension, Freeman, 1977.

[42] Mandelbrot, B.B.: The Fractal Geometry of Nature: Form, Freeman, 1982.

[43] Mañé, R.: On the uniqueness of the maximizing measure for rational maps, Bol. Soc. Bras. Mat., 14(1) (1983).

[44] Martin, R.S.: Minimal positive harmonic functions, Trans. Amer. Math. Soc., 49(1941), 137-172.

[45] Meyn, S., Tweedie, R.: Markov Chains and Stochastic Stability, Springer, Berlin, 1993.

[46] Montel, P. Lecons sur les familles normales de fonctions analytiques. Gauthier-Villars, Paris, 1927. 
[47] Parry, W and Pollicott, M. Zeta functions and the periodic orbit structure of hyperbolic dynamics. Astérisque : Société mathématique de France, 1990.

[48] Parthasarathy, K. R.: Probability measures on metric spaces, Academic Press Inc., New York, 1967.

[49] Pollicot, M., Yuri, M.: Dynamical Systems and Ergodic Theory, Cambridge University Press, Cambridge, 1998.

[50] T. Ransford: Potential Theory in the Complex Plane. Cambridge University Press, 1995.

[51] Resnick, Sidney I. : A probability path, Birkhäuser Boston, 1999.

[52] Ruelle, D.: Statistical Mechanics on a compact set with $Z^{\nu}$ action satisfying expansiveness and specification, Trans. Amer. Math. Soc. 185, 237-252 (1973).

[53] Ross, S. M.: Stochastic Processes, John Wiley \& Sons, Inc., New York, 1996. John Wiley and Sons, 1996.

[54] M. Tsuji, Potential theory in modem function theory, Maruzen, Tokyo, 1959.

[55] Walters, P.: An Introduction to Ergodic Theory, Graduate Text in Mathematics, 79, Springer, Berlin, 1982.

[56] Walters, P.: A variational principle for the pressure of continuous transformations, Amer. J. Math. 17, 937-971 (1976).

[57] Woess, W.: Random walks on infinite graphs and groups - a survey on selected topics, Bull. London Math. Soc. 26 (1994), 1-60.

[58] Yongcheng, Y.: Capacities of Julia Sets of Rational Functions, Acta. Mathematica Sinica, New Series, 1990, Vol. 6, No. 2, 120130. 
[59] A. Zdunik: Harmonic measure on the Julia set for polynomial-like maps. Invent. math. 128, 303-327, (1997). 


\section{Curriculum vitae}

\begin{tabular}{|c|c|}
\hline 25.09 .1969 & Born in Munshigonj, Bangladesh. \\
\hline 1979 & Completed Primary education. \\
\hline 1985 & $\begin{array}{l}\text { Obtained Secondary School Certificate from K. K. } \\
\text { Govt. Institution, Munshigonj. }\end{array}$ \\
\hline 1987 & $\begin{array}{l}\text { Obtained Higher Secondary School Certificate from } \\
\text { Haraganga College, Munshigonj. }\end{array}$ \\
\hline 1993 & $\begin{array}{l}\text { Completed Bachelor of Science(Mathematics) from } \\
\text { Dhaka University, Bangladesh. }\end{array}$ \\
\hline 1995 & $\begin{array}{l}\text { Completed Master of Science(Mathematics) from } \\
\text { Dhaka University, Bangladesh. }\end{array}$ \\
\hline 1997 & $\begin{array}{l}\text { Completed a postgraduate diploma(Mathematics) from } \\
\text { the International Center for Theoretical Physics, Italy. }\end{array}$ \\
\hline 1999 & $\begin{array}{l}\text { Completed Master of Science(Mathematics) from } \\
\text { Goettingen University, Germany. }\end{array}$ \\
\hline
\end{tabular}

